\title{
The role of ghrelin and ghrelin O-acyltransferase in obesity: Targets for treatment
}

\author{
Martin Wellman
}

A thesis submitted to the Faculty of Graduate and Postdoctoral Affairs in partial fulfillment of the requirements for the degree of

\author{
Doctor of Philosophy \\ in \\ Neuroscience
}

Carleton University

Ottawa, Ontario

(C) 2016

Martin Wellman 


\section{Abstract}

Ghrelin is the first identified circulating orexigenic hormone. Following translation of the mRNA to its initial prepropeptide, a variety of posttranslational modifications are performed that allow the peptide to bind to its only known receptor, the growth hormone secretagogue receptor type 1A (GHSR1a). The crucial step in this process is the attachment of an acyl sidechain to the serine- 3 residue, which is accomplished through the activity of the enzyme ghrelin O-acyltransferase (GOAT), ultimately leading to the final product named acylated ghrelin. Acylated ghrelin has been shown to signal through various pathways that promote certain phenotypes associated with obesity, including insulin insensitivity, adiposity, and increased feeding. More recently, ghrelin peptides lacking the acyl sidechain have also been found to have influence in various pathways. Due to these effects, the ghrelin system has become a promising target in the goal of combating the current obesity epidemic. Since its discovery, however, ghrelin has been found to be involved in other pathways important for stress, anxiety, depression, and more, making side-effects associated with ghrelin-targeting pharmaceuticals likely. In order for the development of anti-obesity agents to be successful, a detailed knowledge of the ghrelin system and its interrelationships with others is important, not only to develop compounds to target ghrelin and its counterparts but also to help minimize and foresee complications as a result of side-effects. In the current thesis we examine the machinery of the ghrelin system and seek out to identify various ways that metabolic disorders can be approached pharmacologically. We identify two novel ways to inhibit the adipogenic effect of ghrelin using anti-sense strands targeting central GOAT and the development of our own drug, CF801, which reduces circulating acylated ghrelin levels when given peripherally. Both of these approaches contribute to a reduction in weight gain independent of food intake. By inhibiting the enzyme specifically in the brain, we also identify for the first time a role of central GOAT in promoting adiposity, an effect similar to what is found in the periphery. 


\section{Acknowledgements}

I would like to thank Dr. Alfonso Abizaid, my supervisor at Carleton University, who guided me throughout my graduate degree and acted as an excellent mentor. He provided support and encouragement both in the lab and outside the lab. I would have not been able to finish my degree without his support. I would also like to thank Dr. Zack Patterson for the considerable contributions that he made to this thesis, as well as Dr. Barbara Woodside who I collaborated with early on in my graduate degree when I was just starting to learn the techniques I would use later on, with the guidance of Dr. Elaine Waddington Lamont. I would also like to thank my fellow students who provided support when I needed it most, including Harry MacKay, Zack and Brianne Patterson, Veronique St-Onge, and Warren Caldwell, as well as Rim Khazall and Samantha King. Of course I would also like to thank my friends Andrew Spencer, Claire Hughson, Dominique White, and Oliver, who have been with me through the ups and downs, and my family for their support throughout the years. 


\title{
Abbreviations
}

\author{
5-HT2C 5-hydroxytryptamine 2C receptor \\ ACC acetyl-CoA carboxylase \\ ACL ATP citrate lyase \\ ACTH adrenocoticotropic hormone \\ AgRP agouti-related peptide \\ Ahx amino hexanoic acid \\ AMPK $\quad 5^{\prime}$ AMP-activated protein kinase \\ BSA bovine serum albumin \\ BSX homeobox domain transcription factor \\ CaMKK calmodulin-dependent protein kinase kinase \\ cAMP cyclic adenosine monophosphate \\ CART cocaine and amphetamine regulated transcript \\ CPT1 carnitine palmitoyltransferase 1 \\ $\mathrm{CRH}$ corticotropin-releasing hormone \\ $\mathrm{D}_{1} \mathrm{R}$ dopamine 1 receptor \\ $\mathrm{D}_{2} \mathrm{R}$ dopamine 2 receptor \\ DIO diet-induced obese \\ $\mathrm{DMH}$ dorsomedial nucleus of the hypothalamus \\ DMSO dimethyl sulfoxide \\ ELISA enzyme-linked immunosorbent assay \\ ER endoplasmic reticulum \\ FAS fatty acid synthase \\ FoxO1 forkhead box protein O1 \\ GABA gamma-aminobutyric acid \\ $\mathrm{GH} \quad$ growth hormone \\ GHRH growth-hormone-releasing hormone \\ GHSR growth hormone secretagogue receptor \\ GHSR1a growth hormone secretagogue receptor type 1A \\ GOAT ghrelin O-acyltransferase
}


GPCR G-protein coupled receptor

HPA hypothalamic-pituitary-adrenal

HPLC high-performance liquid chromatography

ICV intracerebroventricular

IP intraperitoneal

$\mathrm{IP}_{3} \quad$ inositol 1,4,5-trisphosphate

IV intravenous

LCFA long-chain fatty acid

$\mathrm{LH}$ lateral nucleus of the hypothalamus

MBOAT membrane-bound O-acyltransferase

MC3R melanocortin 3 receptor

MC4R melanocortin 4 receptor

MCFA medium-chain fatty acid

mGOAT mouse ghrelin $\mathrm{O}$-acyltransferase

MT-II melanotan II

NPY neuropeptide $\mathrm{Y}$

$\mathrm{PC}_{1 / 3}$ prohormone convertase $1 / 3$

pCREB phosphorylated cAMP response-element-binding protein

$\mathrm{PIP}_{2} \quad$ phosphatidylinositol 4,5-bisphosphate

PLC phospholipase C

POMC proopiomelanocortin

PVN paraventricular nucleus of the hypothalamus

RER respiratory exchange ratio

ROS reactive oxygen species

SSRI selective serotonin reuptake inhibitor

SST5 somatostatin receptor subtype 5

Tat trans-activator of transcription

UCP2 uncoupling protein 2

VMH ventromedial nucleus of the hypothalamus

VMO vivo-morpholino antisense oligonucleotide

VTA ventral tegmental area 


\section{Contents}

List of Tables $\quad \mathbf{x}$

List of Figures $\quad$ xi

1 Ghrelin \& Ghrelin O-Acyltransferase 1

1.1 Acylation of ghrelin depends on the enzyme ghrelin O-acyltransferase 2

1.2 Effects of acylated ghrelin . . . . . . . . . . . . . . 4

1.2.1 Ghrelin's role in feeding and obesity . . . . . . . . . 4

1.2.2 Ghrelin's role in food reward . . . . . . . . . . 5

1.2.3 Ghrelin's role in diabetes and glucose control . . . . . . . 6

1.3 Effects of desacyl ghrelin . . . . . . . . . . . . . . . . . . 7

1.4 Signaling pathways of GHSR1a . . . . . . . . . . 8

1.4.1 Ghrelin's effect on NPY/AgRP and POMC signaling . . . . 8

1.4.2 Ghrelin's effect on NPY/AgRP signaling occurs via an AMPK/UCP2-dependent pathway . . . . . . . . . 9

1.4.3 Central ghrelin's effect on peripheral metabolism . . . . . . 12

1.5 Ghrelin and GOAT are highly conserved between species . . . . . 13

1.6 Ghrelin O-acyltransferase and ghrelin are co-expressed in various tissues 14

1.7 Posttranslational processing of the ghrelin gene product produces several peptide hormones . . . . . . . . . . . . . 15

1.8 GOAT acyl substrates are derived from the diet $\ldots \ldots \ldots$

1.9 The active core of ghrelin for receptor and GOAT recognition consists of the first four and five amino acids, respectively . . . . . . . 18

1.9.1 Residue 1: Glycine . . . . . . . . . . . . . . . . 19

1.9.2 Residue 2: Serine . . . . . . . . . . . . . . . . . 19

1.9 .3 Residue 3: Serine . . . . . . . . . . . . . . . . . 19

1.9.4 Residue 4: Phenylalanine . . . . . . . . . . . . . 20

1.9.5 Residue 5 and beyond . . . . . . . . . . . . . 20

1.10 Structure and active site of ghrelin $\mathrm{O}$-acyltransferase . . . . . . . 21 
1.11 GHSR1a has high levels of constitutive activity . . . . . . . . . 22

1.12 GHSR1a signaling potency and timeframe depend on the length of ghrelin's fatty-acyl side chain . . . . . . . . . . . . . 24

1.13 Transport of ghrelin across the blood-brain barrier . . . . . . . . . . 25 25

1.14 Leptin has inhibitory effects on the ghrelin system . . . . . . . . 26

1.15 Development and rearrangement of hypothalamic feeding circuits . . 27

1.16 Obesity is associated with ghrelin resistance and hypothalamic dysregulation . . . . . . . . . . . . . . . 28

2 Pharmacological Agents Targeting the Ghrelin System

2.1 Different phenotypes of ghrelin and GOAT knockout animals are revealed by exposure to high-fat diet . . . . . . . . . . . . . . 31

2.2 Measuring GOAT activity . . . . . . . . . . . . . . 33

2.3 Inhibition of ghrelin O-acyltransferase by the designed drug GO-CoA-Tat . . . . . . . . . . . . 34

2.4 Inhibition of ghrelin O-acyltransferase with designed drugs incorporating a triazole-linked lipid group . . . . . . . . . . . 35

2.5 Treating metabolic disorders with the desacyl ghrelin analog AZP-531 36

2.6 Neutralizing acyl-ghrelin with antibodies . . . . . . . . . 37

2.6.1 Passive immunization against acylated ghrelin . . . . . . . . 37

2.6.2 Hydrolyzing anti-ghrelin antibodies . . . . . . . . . . 39

2.6.3 Active immunization against acylated ghrelin . . . . . . 40

2.7 Neutralizing acyl-ghrelin with a spiegelmer . . . . . . . . . . 41

2.8 Inhibiting GOAT and imitating desacyl ghrelin with CF801 . . . 43

3 Chronic subcutaneous octanoylated Dap3 ghrelin(1-5) does not alter weight gain, metabolism, or glucose tolerance $\quad 46$

3.1 Introduction . . . . . . . . . . . . . . . . 46

3.2 Methods . . . . . . . . . . . . . . . . . . . . 47

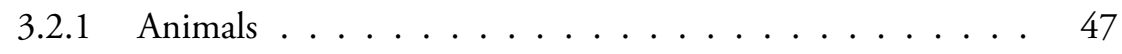

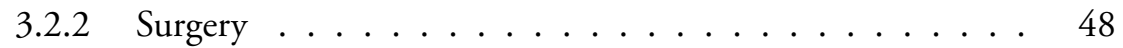

3.2.3 Respiratory exchange ratio . . . . . . . . . . . . 48

3.2.4 Glucose tolerance test . . . . . . . . . . . . . . . 49

3.3 Results ........................... 49

3.3.1 Effect on weight gain and RER in standard chow-fed mice . 49

3.3.2 Effect on weight gain, RER, and glucose tolerance in high-fat diet-fed mice . . . . . . . . . . . . . . . . 49

3.4 Discussion . . . . . . . . . . . . . . . 52

3.4.1 Using a novel approach to reduce GOAT activity . . . . . 53 
4 Novel regulator of acylated ghrelin, CF801, reduces weight gain, rebound feeding after a fast, and adiposity in mice $\quad \mathbf{5 4}$

4.1 Abstract ........................ 54

4.2 Introduction . . . . . . . . . . . . . . 55

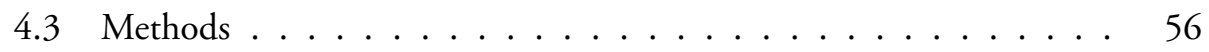

4.3.1 Design of CF801 . . . . . . . . . . . . 56

4.3.2 Cell-based ghrelin secretion assay . . . . . . . . . . 57

4.3.3 Fluorescence-based GOAT activity assay . . . . . . . . 57

4.3 .4 Animals .................... 58

4.3.5 Effects of CF801 on plasma ghrelin concentrations and rebound food intake in fasted mice . . . . . . . . . . . 58

4.3.6 Tissue and blood analysis ............. 58

4.3.7 Effects of CF801 on food intake, weight gain and adiposity . 59

4.3.8 Effects of CF801 on energy expenditure . . . . . . . . 59

4.3 .9 Statistical analyses . . . . . . . . . . . . . 60

4.4 Results . . . . . . . . . . . . . . . . . . 60

4.4.1 CF801 inhibits acyl ghrelin secretion In Vitro and In Vivo . 60

4.4.2 CF801 does not serve as an effective inhibitor of ghrelin octanoylation by GOAT . . . . . . . . . . . . . . 66

4.4.3 CF801 treatment reduces high-fat diet intake and body weight gain without affecting caloric intake . . . . . . 67

4.4.4 CF801 increases the utilization of fat as fuel . . . . . . . 67

4.4.5 CF801 treatment alters body composition . . . . . . 68

4.5 Discussion . . . . . . . . . . . . . . . . . 69

4.5.1 Targeting central GOAT . . . . . . . . . . 71

4.6 Acknowledgements . . . . . . . . . . . . . . . 71

5 Knockdown of central ghrelin O-acyltransferase by vivo-morpholino reduces body mass of rats fed a high-fat diet $\quad 73$

5.1 Abstract ........................ 73

5.2 Introduction . . . . . . . . . . . . . . 74

5.3 Methods . . . . . . . . . . . . . . . . . . . 75

5.3.1 Experiment 1: Effect of food deprivation and food restriction on GOAT, ghrelin, and GHSR1a mRNA in the fundus and the hypothalamus . . . . . . . . . . . . 75

5.3.2 Experiment 2: Effect of central GOAT knock-down on body weight and food intake . . . . . . . . . . . 77

5.4 Results . . . . . . . . . . . . . . . . . 79 
5.4.1 Experiment 1: Effect of food deprivation and food restriction on GOAT, ghrelin, and GHSR1a mRNA in the fundus and the hypothalamus . . . . . . . . . . . . 79

5.4.2 Experiment 2: Effect of central GOAT knock-down on body weight and food intake ................. 81

5.5 Discussion . . . . . . . . . . . . . 83

6 General Discussion $\quad 86$

6.1 Elaborating on the central effects of GOAT . . . . . . . . . . . 86

6.2 Anti-GOAT VMOs, CF801, and desacyl ghrelin $\ldots \ldots . . . .887$

6.3 Potential barriers in human translation . . . . . . . . . . . . 88

6.4 Conclusion . . . . . . . . . . . . . . . 89

$\begin{array}{lr}\text { Appendices } & 91\end{array}$

A Copyright Documentation $\quad \mathbf{9 2}$

A.1 Permission from Frontiers in Endocrinology . . . . . . . . . . . . 92

A.2 Permission from Peptides . . . . . . . . . . . . 93

A.3 Co-author permission statements . . . . . . . . . . . 94

$\begin{array}{lc}\text { Bibliography } & 103\end{array}$ 


\section{List of Tables}

5.1 Primer sequences for qRT-PCR . . . . . . . . . 77 


\section{List of Figures}

1.1 Acylation of ghrelin by ghrelin $\mathrm{O}$-acyltransferase . . . . . . . . . . . . 3

1.2 GHSR1a signaling through the AMPK pathway . . . . . . . . . 10

1.3 Acylated ghrelin synthesis and secretion . . . . . . . . . . . 16

1.4 Amino acid properties important for GOAT recognition $\ldots \ldots . .18$

3.1 Effect of Dap3-ghrelin(1-5) on weight gain and metabolism on standard chow . . . . . . . . . . . . . . 5 50

3.2 Effect of Dap3-ghrelin(1-5) on weight gain, metabolism, and glucose tolerance on a high fat diet . . . . . . . . . . . 51

4.1 CF801 inhibits acyl ghrelin secretion from SG-1 cells following 24 hours of incubation . . . . . . . . . . . . 61

4.2 Effect of CF801 on plasma acylated ghrelin, chow consumed, and blood glucose . . . . . . . . . . . . . . . 62

4.3 Effect of CF801 on hGOAT and mGOAT activity in Sf9 cells . . . . 63

4.4 Effect of CF801 on caloric intake . . . . . . . . . . . . 63

4.5 Effect of CF801 on food intake and weight gain . . . . . . . . . 64

4.6 Effect of CF801 metabolism and body composition . . . . . . . 65

5.1 qRT-PCR fold changes in the hypothalamus and fundus in response to food deprivation and food restriction . . . . . . . . 80

5.2 Circulating acylated ghrelin levels in response to food deprivation and food restriction . . . . . . . . . . . . . 81

5.3 Effects of knockdown of central GOAT by anti-sense administered to the lateral ventricles . . . . . . . . . . . . . . . 82 


\section{Chapter 1}

\section{Ghrelin \& Ghrelin O-Acyltransferase}

The recent surge in obesity rates has been largely associated with the more affluent nations, particularly those in the Western world. Despite this, according to the World Health Organization, a growing trend in overweight and obesity is beginning to emerge in lower-income developing nations as well; obesity extends well beyond the walls of the more affluent nations, afflicting individuals throughout the world at all levels along the socioeconomic gradient (1). Concomitant with obesity is an elevated risk of hypertension, type 2 diabetes, hypercholesterolemia, coronary heart disease, stroke, asthma, arthritis, and cancer $(1-4)$. Worldwide figures from the past decade provided by the World Health Organization show sobering statistics, with over 1.4 billion adults and over 40 million children under the age of five being overweight (1). Recent trends in the food industry have seen an increase in highly processed, high calorie and low cost sugars and fats. While a push at the consumer as well as government levels at managing these obesogenic agents has emerged, we have as of yet not seen any significant impact on reducing obesity rates; indeed, rates continue to rise (1). While the pharmaceutical industry has shown great interest in tapping this lucrative segment of the market, weight loss agents and drugs to treat metabolic disorders in the form of receptor blockers, reuptake inhibitors, enzyme inhibitors, and others have shown little benefit with often costly side effects, amongst those mood disorders, anxiety, and suicidal ideation $(5-8)$.

Currently, a promising area of research in the field of metabolic disorders centers around ghrelin, a 28 -amino acid hormone produced primarily by the stomach and the upper intestine, as well as by the liver, kidneys, pancreas, lung, heart, testis, pituitary, and hypothalamus (9-16). Ghrelin was initially identified as a growth hormone (GH) secretagogue acting on the pituitary (9). It was identified as the ligand for the 
growth hormone secretagogue receptor type 1A (GHSR1a) in 1999 by Kojima and colleagues from purified rat stomach (9) and is the only known peripherally circulating peptide hormone that promotes food intake. In addition to its role in feeding behavior, ghrelin promotes adiposity by switching energy utilization to favor breakdown of carbohydrates instead of fats $(17,18)$. As will be discussed, ghrelin's roles both peripherally and centrally are diverse and widespread, affecting insulin sensitivity and glucose control, the cardiovascular system, anxiety, depression, and more. An important component of the ghrelin system is the enzyme ghrelin $\mathrm{O}$-acyltransferase (GOAT). This enzyme catalyzes the addition of a fatty acyl sidechain, typically but not exclusively involving an 8 carbon octanoyl-CoA, derived from dietary medium-chain fatty acids (MCFAs), onto the serine-3 residue of the ghrelin peptide, resulting in acylated ghrelin $(19,20)$. Without this modification, ghrelin in its desacyl (unacylated) form cannot bind to its receptor. Less than $10 \%$ of circulating ghrelin is acylated (21, 22). It has been shown that much of ghrelin's activity, including its effect on feeding and adiposity, rely on this octanoyl modification. As such, the GOAT enzyme has been the center of much research, particularly in terms of GOAT inhibitors to reduce acylated ghrelin levels, which one would hope would lead to a reduction in obesity and improvement in diabetes. Along with these inhibitors, progress has been made in developing and identifying GHSR1a antagonists and desacyl ghrelin analogs, often with promising but mixed results.

In this thesis, I will give an overview of the ghrelin system, the effects this peptide has in both its acylated and desacylated forms, and the roles that GOAT and ghrelin play in the body, particularly in relation to various metabolic disorders. During the discussion, I hope to point out areas in which pharmaceuticals targeting the ghrelin system may be beneficial with hints as to the effects that such compounds would have on physiology, leading up to the development of our own peptide-based drug, CF801, designed to reduce acylated ghrelin levels leading to reduced fat mass and improved metabolic phenotype.

\subsection{Acylation of ghrelin depends on the enzyme ghrelin O-acyltransferase}

GOAT is a member of the membrane-bound O-acyltransferases (MBOATs) and is necessary for the post-translational activation of the ghrelin molecule by catalyzing the addition of a fatty acid to the serine-3 residue of proghrelin (see Figure 1.1) $(9,13$, $19,20,23-26)$. The resulting ester bond is relatively unstable, and along with high levels of esterases and other enzymes contributing to hydrolysis, results in a relatively short in vivo half-life of 9 to 13 minutes $(27,28)$. This acylation, typically but not exclusively involving eight-carbon fatty acid-CoAs, is required for ghrelin's ability to 


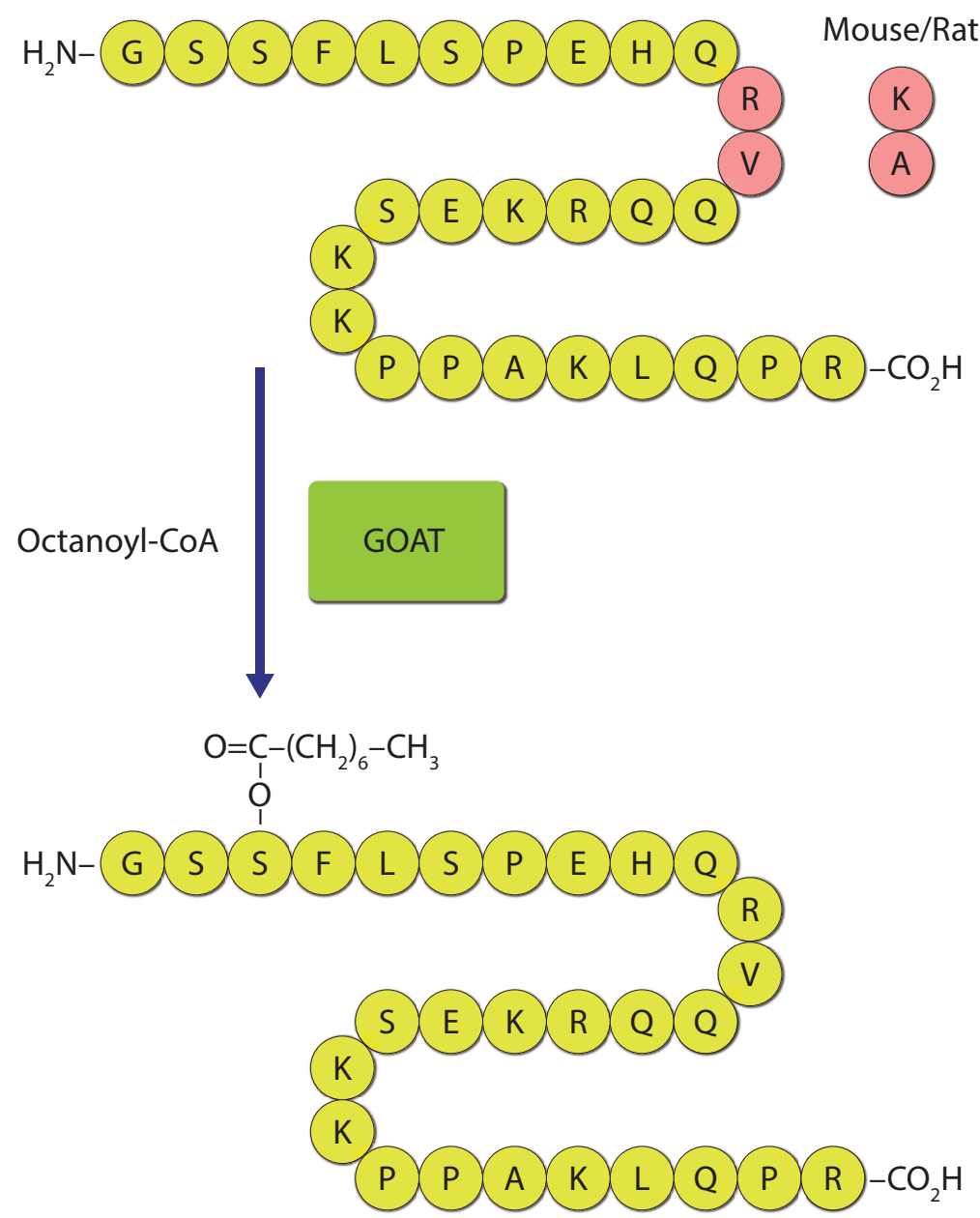

Figure 1.1: Acylation of ghrelin by ghrelin $\mathrm{O}$-acyltransferase.

The mature ghrelin peptide is a string of 28 amino acids. Acylation involves attachment of a fatty acid to the serine- 3 residue through the action of the enzyme GOAT. The source of the fatty acid is fatty acyl-CoAs derived from the diet. While acylation is typically associated with 8-carbon octanoyl CoA, fatty acids of different lengths can be attached. Acylation actually involves a proghrelin peptide with a C-terminal tail sequence which is cleaved following attachment of the side chain. As depicted above, human ghrelin differs from rat and mouse ghrelin by its eleventh and twelfth amino acids. 
signal through its only known receptor, the GHSR1a. It is believed that ghrelin is the only protein that undergoes acylation by octanoate, and GOAT is the only known enzyme that acylates ghrelin, making GOAT a desirable pharmacological target to reduce acylated ghrelin levels $(19,20)$.

\subsection{Effects of acylated ghrelin}

Since its discovery, acylated ghrelin has been found to have a growing number of effects in various tissues. This peptide has been found to inhibit glucose-stimulated insulin release from pancreatic cells (29-36), stimulate gastric acid secretion and motility (3740 ), modulate stress and anxiety (41-47), affect cardiovascular function (48-51), promote slow-wave sleep in humans (52), promote proliferation of adrenal cells (53), aid in survival during prolonged caloric restriction $(54,55)$, and enhance immune response likely through its GH-stimulating activity (56). It is important to take into consideration this wide array of processes that ghrelin is involved in when examining the use of GOAT inhibiting drugs, GHSR1a agonists and antagonists, or desacyl ghrelin analogs. As with any anti-obesity drug to date, one would expect considerable side effects as a result of targeting the ghrelin system. An analysis of the costs and benefits comes into play with any pharmaceutical compound. Irrespective of a drug's efficacy in obesity, diabetes, and related treatments, such a drug would prove beneficial in the laboratory to address more academic issues surrounding ghrelin, GOAT, and related metabolic processes to further expand our knowledge surrounding this complex system. In the following sections, I will introduce some of the roles ghrelin plays that may represent important etiological factors or pharmacological targets in metabolic disorders.

\subsubsection{Ghrelin's role in feeding and obesity}

Much of the interest surrounding the ghrelin system is its ability to promote adiposity through various mechanisms. Plasma ghrelin levels increase preprandially $(57,58)$ and decrease postprandially during satiety $(57,59)$. Exogenous administration of acylated ghrelin has been found to increase food intake when administered peripherally in rats $(17,60,61)$, mice $(17,18)$, and humans $(62)$. Similarly, central administration in rats also causes increases in food intake and body weight gain $(17,63-66)$ along with increased feed efficiency (67). This increased weight gain is a result of increased fat mass without changes in lean body mass (67). Peripheral and central ghrelin administration also causes a shift in substrate utilization away from fats to carbohydrates $(17,18,67)$, thereby protecting fat stores. Adiposity is further promoted by causing a shift in preference to fatty foods when given both centrally (68) and peripherally (69). Details on the specifics of ghrelin in relation to feeding and adiposity, including the intracellular signaling cascades responsible for these effects, will be discussed in later 
sections.

\subsubsection{Ghrelin's role in food reward}

Feeding is a necessity providing us with the energy, nutrients, and the building blocks required to sustain life. In order to provide the drive to seek out food, feeding has evolved to be highly rewarding. Indeed, we often eat beyond our minimal homeostatic needs as a form of comfort food for the simple pleasure, or, in some cases, to relieve stress (for a review, see (70)). This hedonic feeding arises from food's ability to activate the reward pathways associated with the mesolimbic dopaminergic system and is the catalyst to many instances of obesity. Within this system, ghrelin has been shown to play a critical stimulatory role. Demonstrating this, peripheral administration of ghrelin to wild-type mice increases preference and intake of highly-palatable food, particularly a high-fat diet $(69,71)$. These data are supported by results of a free choice paradigm, in which both regular chow and a highly palatable food are provided (71), as well as in operant conditioning and conditioned-place preference experiments $(18,69,71,72)$.

Similarly, direct injection of ghrelin bilaterally into the ventral tegmental area (VTA) causes an increase in intake of highly palatable food (71). While Abizaid and colleagues (73) have shown that direct VTA infusion of ghrelin causes an increase in feeding on standard chow, Egecioglu et al. (71) were not able to demonstrate this unless a highly palatable food source was provided. Supporting their results, Egecioglu et al. (71) found that lesioning the VTA, while still providing ghrelin administration, has no effect on standard chow intake but significantly reduces intake of highly rewarding food. Further demonstrating the VTA's role in ghrelin-induced feeding, Abizaid et al. (73) demonstrated that intra-VTA administration of a ghrelin antagonist block's peripheral ghrelin's orexigenic effect. Interestingly, ghrelin's ability to increase feeding is absent in orexin-deficient mice as well as in wild-type mice given an orexin 1 receptor antagonist (69). Inhibition of ghrelinergic signaling by intraperitoneal administration of JMV2959, a GHSR1a antagonist, reduces preference for rewarding food in a free choice paradigm, with similar results in GHSR KO animals (71). This may indicate a dampening of hedonic feeding, allowing homeostatic mechanisms to function with less interference from over-riding reward systems.

At the receptor level, GHSR1a and the dopamine 1 receptor $\left(D_{1} R\right)$ form heterodimers, and when both receptors are bound simultaneously by their respective ligands a four-fold amplification of $\mathrm{D}_{1} \mathrm{R}$-associated cyclic adenosine monophosphate (cAMP) accumulation occurs (74). These two receptors are co-expressed in midbrain reward areas including the ventral tegmental area (74). Given that acylated ghrelin levels rise preprandially during periods of hunger, one can postulate that acylated ghrelin can contribute to the rewarding aspect of feeding via GHSR $1 \mathrm{a}-\mathrm{D}_{1} \mathrm{R}$ heterodimer for- 
mation. Ghrelin in the VTA also increases synapse formation and dopamine turnover in the nucleus accumbens (73), and GHSR1a knockout mice do not show dopamine release in the nucleus accumbens when given a highly palatable food (71).

\subsubsection{Ghrelin's role in diabetes and glucose control}

Type II diabetes is a serious medical condition characterized by insulin resistance and high blood glucose levels. In 2014, it was estimated that $9 \%$ of the world-wide population aged 18 or older had diabetes, with 1.5 million deaths in 2012 (75). 90\% of the population that has diabetes have type II, which is mainly the result of obesity and inactivity (75). Consequences include damage to the heart, blood vessels, eyes, kidneys, and nerves (75), with $50 \%$ of individuals with diabetes dying of cardiovascular disease (76). These statistics clearly demonstrate the importance of improved treatment and prevention of diabetes. Numerous studies to date have demonstrated ghrelin's role in insulin sensitivity and glucose control, with beneficial effects resulting from reduced acylated ghrelin levels.

Insulin is secreted from the beta cells of the pancreas and stimulates uptake of circulating glucose into cells of skeletal muscle and fat tissue for use as an energy source. Both ghrelin and GHSR1a have been detected in the pancreatic islets $(30,77,78)$. Ghrelin stimulates plasma glucose levels and decreases plasma insulin levels in humans, mice, and rats $(30,79-81)$. The onset of these effects are rapid, with elevations in glucose occurring within 15 minutes and decreases in insulin within 30 minutes after intravenous (IV) ghrelin administration in humans (79). Ghrelin also inhibits glucose-stimulated insulin secretion in isolated rat (33) and mouse (34) pancreas. Intraperitoneal (IP) injection of GHSR1a antagonists cause a decrease in fasting glucose levels and improves insulin response in a glucose tolerance test in mice (30). Ghrelin's ability to increase plasma glucose appears to be through attenuation of glucose-induced insulin release, by way of enhancing delayed outward $\mathrm{K}^{+}$currents and decreasing intracellular $\mathrm{Ca}^{2+}$ concentrations in $\beta$-cells, an effect dependent on $\mathrm{G}_{\mathrm{i} / \mathrm{o}}$ signaling. Interestingly, canonical GHSR1a signaling occurs through a $G_{q}$ pathway, suggesting that GHSR1a intracellular signaling is at least partially modified in pancreatic $\beta$-cells. Indeed, heterodimerization of GHSR1a with somatostatin receptor subtype 5 (SST5) alters GHSR1a signaling by switching G-coupling to a $\mathrm{G}_{\mathrm{i} / \mathrm{o}}$-dependent pathway that suppresses cAMP accumulation (82). Heterodimerization is favored when the ghrelin:somatostatin ratio is elevated, while a low ratio reduces heterodimerization and allows return to canonical $\mathrm{G}_{\mathrm{q}}$ signaling (82). These data suggest that during periods of low energy availability, when endogeneous acylated ghrelin levels are high, ghrelin signaling suppresses $\beta$-cell activity and hence insulin release. This response may seem unusual in that high ghrelin levels have been associated with reductions in fat utilization and an increase in carbohydrate utilization, whereas ghrelin-induced reduc- 
tions in insulin may promote a reduction in cellular uptake of carbohydrates as an energy source. This may be a mechanism to reduce energy utilization by the periphery while providing adequate energy input in the form of carbohydrates to the brain. Similarily, growth hormone secretagogue receptor (GHSR)-null mice, while having normal blood-glucose levels when given ad lib access to chow, have abnormally low blood-glucose during caloric restriction (83). Re-expression of GHSR specifically in agouti-related peptide (AgRP) neurons restores blood glucose to normal levels, an effect attributed to increases in glucagon as well as mRNA for various genes associated with hepatic gluconeogenesis (83).

\subsection{Effects of desacyl ghrelin}

It was originally believed that desacyl ghrelin, which cannot bind to GHSR1a, was physiologically inactive and represented a waste product of acylated ghrelin. As such, much less is known regarding the effects of the unacylated vs. the acylated form of ghrelin. It is now becoming more apparent that desacyl ghrelin has a role separate to that of acylated ghrelin. In 2004, Broglio and colleagues (84) demonstrated that intravenous co-administration of desacyl ghrelin along with acylated ghrelin in humans abolished the latter's ability to increase plasma glucose and decrease insulin levels. This antagonistic role, however, does not always generalize to other effects of acylated ghrelin, since desacyl ghrelin has no effect on ghrelin's $\mathrm{GH}$, prolactin, adrenocoticotropic hormone $(\mathrm{ACTH})$, and cortisol responses (84). Furthermore, desacyl ghrelin administered alone has no effect on these parameters, suggesting that in this case it acts to inhibit the effects of ghrelin rather than induce effects on its own, or that perhaps the two interact in a novel way (84). Additionally, it has been demonstrated that desacyl ghrelin reduces acylated ghrelin's suppression of pancreatic polypeptide from isolated mouse pancreatic islets (85), inhibits ghrelin's orexigenic effects when peripherally coinjected in rats (86), inhibits feeding in food-deprived mice when administered alone ICV or IP (87), improves the acylated ghrelin-induced insulin insensitivity when coadministered with acylated ghrelin in humans with adult-onset GH deficiency (88), and inhibits glucose output by primary hepatocytes (89).

In obese subjects with type 2 diabetes, overnight desacyl ghrelin infusion reduces circulating acylated ghrelin levels, increases the desacyl:acyl ghrelin ratio, and decreases postprandial glucose levels, along with an increase in insulin sensitivity (90). Desacyl ghrelin's ability to reduce acylated ghrelin levels suggests some form of negative feedback, although such a mechanism has not yet been addressed. Based on these data, it may be that the desacyl:acyl ghrelin ratio may be an important modulator of both glucose control and insulin secretion. Whether these two forms of ghrelin compete for binding at an unknown receptor is not known. In addition to studies examining 
exogenous administration of desacyl ghrelin, Zhang et al. (91) overexpressed ghrelin in adipose tissue and found elevations in desacyl ghrelin, but not acyl ghrelin, along with improved insulin sensitivity and glucose tolerance, as well as reduced white adipose tissue. The lack of an increase in acylated ghrelin is particularly interesting, as it suggests that GOAT activity or expression is not modulated by ghrelin expression. It furthermore suggests that acylation of ghrelin is not a required step in the enzymatic pathway leading to endogenous ghrelin synthesis.

In a clinical study, Benso et al. (92) demonstrated the beneficial metabolic effects of continuous overnight infusion of desacyl ghrelin in humans. In subjects given desacyl ghrelin over a 16 hour period, from $9 \mathrm{pm}$ to $1 \mathrm{pm}$ the next day, with a well controlled dinner at $9 \mathrm{pm}$ and breakfast at 9 am, overall reduced glucose levels, as measured by area under the curve, were observed during this time period (92). While overall insulin concentrations as measured by area under the curve did not differ over the 16 hour time-frame, desacyl ghrelin induced a significant increase in insulin levels for the 60 minute periods immediately following both dinner and breakfast (92), demonstrating improved glucose-stimulated insulin release. Furthermore, free fatty acid areas under the curve decreased by $52 \%$ in desacyl ghrelin-treated subjects (92).

These data are very promising when considering desacyl ghrelin analogs or GOAT inhibitors as pharmacological agents. Reductions in body mass as well as improved insulin sensitivity and glucose control are three major components of what one would expect from effective treatment of many metabolic disorders, or in particular, obesity and type 2 diabetes. While for the most part desacyl ghrelin shows different, often opposite, effects as acyl ghrelin, this is not always the case. For example, both desacyl and acyl ghrelin inhibit cell death in cardiomyocytes and endothelial cells (48) and act as vasodilators in humans (93). Whether this overlap is a result of an unknown shared receptor is still not known, but remains a fascinating possibility. It is important to keep in mind, however, that in some cases these similar effects may be a result of desacyl ghrelin signaling exclusively, as acylated ghrelin quickly degrades to its desacyl form.

\subsection{Signaling pathways of GHSR1a}

\subsubsection{Ghrelin's effect on NPY/AgRP and POMC signaling}

Neuropeptide Y (NPY)/AgRP and proopiomelanocortin (POMC) neurons are key components of the central feeding system, with NPY/AgRP providing strong orexigenic control and POMC acting as a precursor to various anorectic peptides. These neurons, primarily located within the arcuate nucleus, are often termed first-order sensory neurons for the homeostatic control of food intake. The ghrelin system has often been described as a sensing mechanism to detect humoral signals related to energy 
balance, including hormones, glucose, and fatty acids (94). Ghrelin-containing neurons have been identified in the hypothalamus, in areas surrounding the third ventricle including the arcuate nucleus $(83,95-97)$. Within the arcuate, excitatory ghrelinergic projections innervate orexigenic NPY and $\mathrm{AgRP}$ neurons, increasing mRNA for both as well as stimulating their release $(63,64,83,95,96,98,99)$. These neurons subsequently project to the paraventricular nucleus of the hypothalamus (PVN), and innervation by ghrelinergic terminals is also found in the dorsomedial, lateral, and paraventricular nuclei of the hypothalamus (95). Mice with a single knock-out to either NPY or AgRP (but not both) still respond to ghrelin by increasing regular chow intake, although this response is slightly attenuated compared to wild-type animals (100). On the other hand, NPY/AgRP double knock-outs are completely absent in their orexigenic response to ghrelin (100). These data suggest redundancy within the two systems.

Additionally, projections originating from NPY/AgRP neurons in the arcuate stimulate inhibitory gamma-aminobutyric acid (GABA)ergic projections onto POMC neurons, reducing the anorectic activity of the POMC/cocaine and amphetamine regulated transcript (CART) system $(95,101)$. In the PVN, ghrelin axons also innervate corticotropin-releasing hormone (CRH)-releasing neurons, which stimulate activity of the hypothalamic-pituitary-adrenal (HPA) axis (95). Interestingly, systemic administration of ghrelin has also been found to increase hypothalamic activity (102-104), with the peripheral signal likely originating in the vagus nerve (105-107).

\subsubsection{Ghrelin's effect on NPY/AgRP signaling occurs via an AMPK/UCP2-dependent pathway}

The evolutionarily conserved serine/threonine kinase 5' AMP-activated protein kinase (AMPK) is a heterotrimeric enzymatic complex that provides a link between nutritional availability and cellular energy reserves and is the route by which ghrelin acts on NPY/AgRP within the arcuate nucleus. Its high level of conservation demonstrates the importance of the AMPK pathway. It is found in cells throughout the body, including within the brain $(101,108-110)$. Central AMPK activity in hypothalamic regions is increased by ghrelin and inhibited by various anorectic signalers including leptin, insulin, glucose, and the melanocortin 3/4 agonist melanotan II (MT-II) (111). AMPK also responds to changes in the AMP/ATP ratio, with higher ratios leading to increased AMPK activity and subsequent activation of catabolic pathways and inhibition of biosynthetic pathways to favor ATP accumulation (112) (for reviews, see (113, 114)).

Ghrelin signaling on NPY/AgRP neurons, as depicted in Figure 1.2, begins with activation of GHSR1a, which is coupled to a $\mathrm{G} \alpha_{\mathrm{q}}$ pathway (101). Following activation, phospholipase $\mathrm{C}$ (PLC) is recruited to the membrane leading to production of 


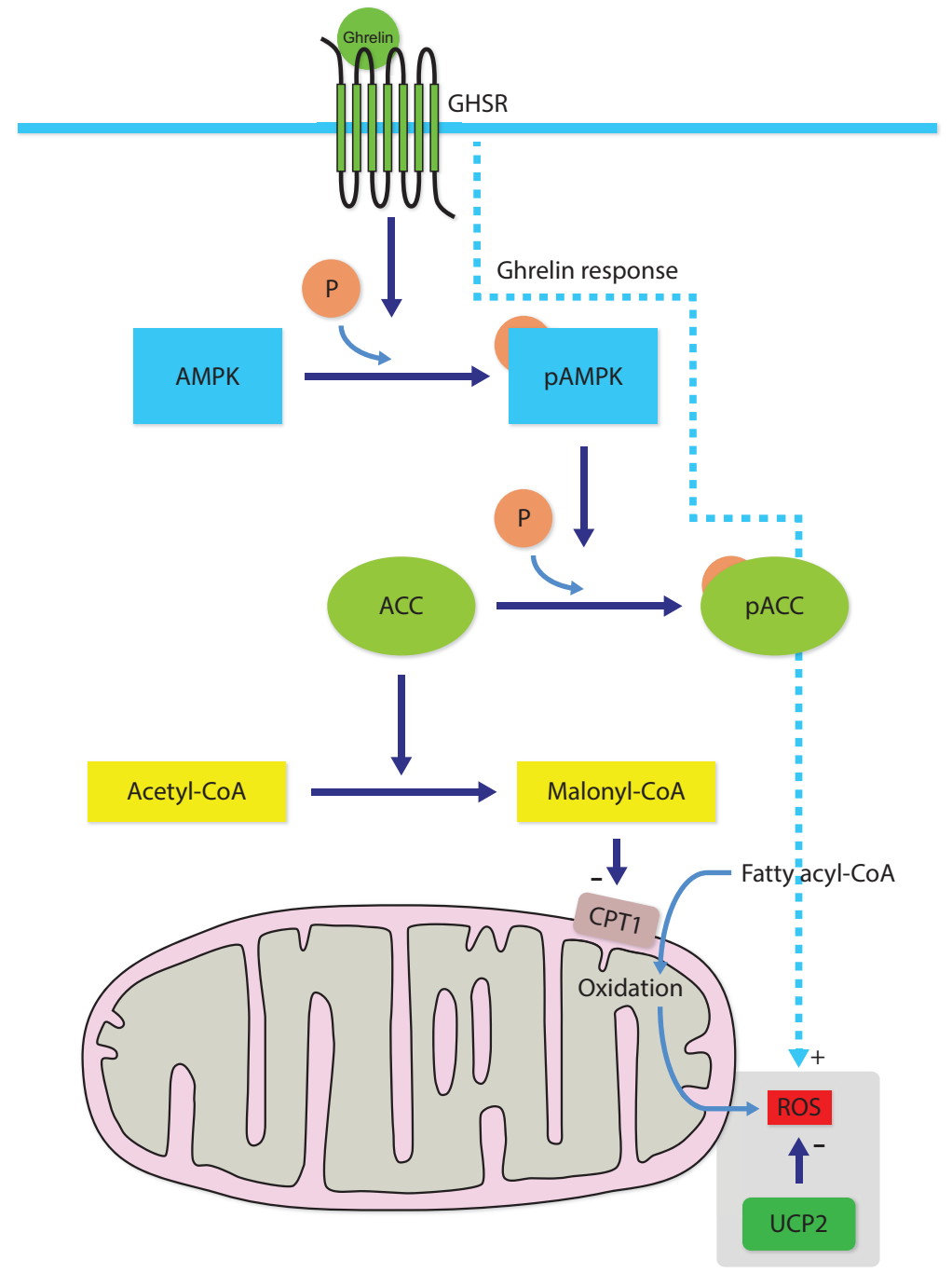

\section{Figure 1.2: GHSR1a signaling through the AMPK pathway.}

In this pathway, depicting the GHSR1a response in NPY/AgRP neurons, unphosphorylated ACC catalyzes the conversion of acetyl-CoA to malonyl-CoA, which inhibits CPT1 activity. By phosphorylating and inactivating ACC, GHSR1a signaling reduces malonyl-CoA synthesis and hence releaves the inhibition on CPT1, thus promoting mitochondrial uptake and oxidation of fatty acyl-CoAs. This oxidation leads to elevations in reactive oxygen species, which promotes UCP2 production to scavenge the reactive oxygen species. This elevation in UCP2 allows the continued oxidation of fatty acids by reducing the oxidative load on the cell, providing a suitable environment for prolonged excitation of NPY/AgRP neurons. 
inositol 1,4,5-trisphosphate $\left(\mathrm{IP}_{3}\right)$ from phosphatidylinositol 4,5-bisphosphate $\left(\mathrm{PIP}_{2}\right)$ (101). $\mathrm{IP}_{3}$ binds to receptors on the endoplasmic reticulum (ER) to cause release of $\mathrm{Ca}^{2+}(101)$. In response to elevations in intracellular $\mathrm{Ca}^{2+}$ levels, calmodulin activates calmodulin-dependent protein kinase kinase (CaMKK) which leads to phosphorylation and activation of AMPK $(101,110,115)$. This activated pAMPK subsequently catalyzes the phosphorylation and inactivation of acetyl-CoA carboxylase (ACC) (101, 110, 115). ACC controls the conversion of acetyl-CoA to malonyl-CoA, which in turn acts as an allosteric inhibitor on carnitine palmitoyltransferase 1 (CPT1) (101). CPT1 acts as a rate-limiting step in fat oxidation by controlling entry of long-chain fatty acids (LCFAs) into mitochondria as a substrate for energy production (101). By inhibiting this pathway via phosphorylation of ACC, ghrelin-induced reductions in malonylCoA remove the inhibition on CPT1, which then promotes uptake and oxidation of LCFAs by mitochondria (101). This pathway promotes ATP production when energy levels are low. As a result of this oxidation, reactive oxygen species (ROS) production increases, which leads to increased expression of uncoupling protein 2 (UCP2) (101). This elevation of UCP2 helps to scavenge ROS to enable further uptake and oxidation of LCFAs and to enable continued excitation of NPY/AgRP neurons. The vast majority (over 90\%) of UCP2-expressing neurons co-express GHSR1a (101). Reducing malonyl-CoA levels via more direct routes such as through the use of ACC inhibitors induces feeding while increasing malonyl-CoA levels reduces feeding (116). Intuitively, reductions in malonyl-CoA are also found during fasting and conversely increase when satiated (116). Along with these effects, signaling through UCP2 promotes mitochondrial biogenesis in NPY/AgRP neurons, which is believed to more than compensate for the reduced mitochondrial capacity to produce ATP due to the uncoupling of oxidative phosphorylation (94).

In UCP2 KO mice, ghrelin's ability to increase food intake by activating NPY/AgRP neurons is attenuated when administered intraperitoneally or when injected into the mediobasal hypothalamus or directly into the VTA (101). This attenuation can be alleviated by administering a ROS scavenging cocktail intracerebroventricularly (101). Furthermore, UCP2 KO mice show increased ROS in NPY neurons in response to ghrelin, an effect not observed in wild-type mice (101). Given that UCP2 KO mice do not show the hyperphagia observed in wild-type mice when given ghrelin, the ability to induce UCP2 and maintain reactive oxygen species at manageable levels appears important in maintaining ghrelin's prolonged orexigenic effect through NPY/AgRP, and to allow continued excitation of these neurons. Through its uncoupling activity, UCP2 also reduces the mitocondrial membrane potential, an effect suggested to possibly contribute to the altered oxygen consumption observed in animals given injections of ghrelin (101). In order to feed this mechanism, IP administration of ghrelin leads to increases in LCFA-CoAs in the hypothalamus, which remain elevated up to 3 hours post-injection with a mild, 
non-significant decline after one hour (101). This decline has been suggested to occur as a result of increased uptake of LCFAs by mitochondria (101). The source of these LCFAs is believed to be from non-esterified fatty acids in circulation which remain elevated 18 hours after injection (101).

Within the arcuate nucleus, where ghrelin regulates NPY/AgRP expression, it is believed that increases in transcription of NPY is mediated by interactions between homeobox domain transcription factor (BSX) and phosphorylated cAMP responseelement-binding protein (pCREB), while AgRP transcription is induced by interactions between BSX and forkhead box protein O1 (FoxO1) (99, 117-121). Elevations in BSX occur downstream of $\beta$-oxidation, which can be blocked by the CPT1 inhibitor etomoxir (99). In contrast, both leptin and insulin promote formation of the inactive phosphorylated form of FoxO1, which leads to decreases in AgRP expression $(118,122)$ and increases in POMC expression $(123-125)$.

Interestingly, GABAergic projections from NPY/AgRP neurons onto POMC neurons display synaptic plasticity in response to ghrelin, promoting an increase in inhibitory and a decrease in excitatory synapses (101), helping to prolong inhibition of POMC neurons and hence reduce their anorectic activity. Andrews et al. (101) point out that at satiety, POMC neurons show elevated baseline ROS levels, an indication that POMC neurons may be preferentially using carbohydrates as an energy source, since carbohydrate utilization generates more ROS. This increase, which is greater than levels found in NPY/AgRP neurons, make POMC neurons more susceptible to the deleterious effects of reactive oxygen species. On top of this, the lack of GHSR1a co-expression with POMC suggests that ghrelin's ability to increase UCP2, and hence protect against oxidative stress, is absent in these neurons. Andrews and colleagues (101) suggest that in the long-term POMC neurons show increased impairment and cell death due to elevated ROS, while NPY/AgRP neurons show resistance. Additionally, ghrelin increases mitochondrial number in NPY neurons, an effect not seen in UCP2 KO mice (101). This increase appears to involve de novo mitochondrial biogenesis, and newly-formed mitochondria generally show improved efficiency and reduced ROS production. This, along with the inhibitory synaptic plasticity observed on POMC neurons, suggest that over time the balance is more likely to shift in favor of orexigenic signaling pathways over anorectic pathways. Despite this, however, obesity has been associated with resistance to ghrelin's effects on NPY/AgRP neurons, an effect that will be discussed shortly.

\subsubsection{Central ghrelin's effect on peripheral metabolism}

As discussed, following intracerebroventricular (ICV) administration of ghrelin, $\beta$ oxidation is promoted centrally. The effects of centrally administered ghrelin on the periphery, however, appear to be quite different with a shift towards fat storage, likely me- 
diated by the hypothalamus (63). Indeed, mRNA in white adipocytes for fat storagepromoting enzymes increase, including those for lipoprotein lipase, ACC $\alpha$, fatty acid synthase (FAS), and stearoyl-CoA desaturase-1 (67). In contrast to central signaling, mRNA levels of CPT $1 \alpha$, the rate-limiting step in fat oxidation, is reduced in white adipocytes (67). Additionally, in brown adipocytes, mRNA for the thermogenesisrelated enzymes UCP1 and UCP3 decrease (67). Glucose utilization in both white and brown adipose tissue increase in a feeding-independent manner, with no changes in insulin sensitivity or plasma insulin, glucose, corticosterone, leptin or free fatty acids (67). These changes in the various enzymes are partly independent of ghrelin's hyperphagic effect, as they are also observed in ICV ghrelin-treated animals that are pair-fed to saline controls, although to a slightly lesser extent (67). These data suggest that central ghrelin primes and promotes peripheral tissue to accept and store incoming fat.

Theander-Carrillo et al. (67) demonstrate that these results are absent in transgenic mice with an impaired sympathetic nervous system lacking $\beta_{1^{-}}, \beta_{2^{-}}$, and $\beta_{3^{-}}$ adrenoreceptors. Given that $\mathrm{AgRP}$ is an antagonist for the melanocortin 3 receptor (MC3R) and the melanocortin 4 receptor (MC4R) (126), and that these melanocortin receptors increase sympathetic outflow and blood pressure (54), these changes might be mediated by central ghrelin's ability to increase AgRP expression and ultimately to reduce sympathetic nervous system activity.

\subsection{Ghrelin and GOAT are highly conserved between species}

There is $90 \%$ amino-acid similarity between mouse, rat, and human GOAT, and 60\% similarity between mammalian and zebrafish GOAT (19). This high conservation among species is also demonstrated by the ability of zebrafish GOAT to acylate human ghrelin (19). On the ghrelin end, human and rodent peptides differ by only two residues $(127,128)$, with the $\mathrm{N}$-terminal 10 amino acids being fully conserved among all mammals examined (127) (see Figure 1.1). Additionally, homologs have been identified in frogs $(129,130)$, chicken $(131)$, and a variety of fish species $(132-136)$. While this high level of conservation suggests that drugs that inhibit non-human GOAT are likely to also inhibit human GOAT, whether this is true or not, and whether different effects of GOAT-inhibition occur in humans, is by no means a question that should be ignored. 


\subsection{Ghrelin O-acyltransferase and ghrelin are co-expressed in various tissues}

mRNA for GOAT is primarily found in the stomach, pancreas, intestine, and pituitary, with small amounts found in other tissues such as the colon and testis $(19,20$, 137). Western blot and immunofluorescence have detected GOAT in the plasma and the anterior pituitary gland (138), although plasma GOAT is likely inactive. Within the brain, however, barely detectable levels of GOAT mRNA have been found (19). Nevertheless, our lab and others have measured GOAT mRNA in the hypothalamus $(26,137,139)$, suggesting that if indeed GOAT protein is present centrally it likely has a role in homeostatic and feeding mechanisms. Stomach levels of GOAT mRNA are approximately 200 -fold lower than those of ghrelin mRNA (20). In general, there is considerable overlap in the distribution of ghrelin and GOAT mRNA, with especially high levels of ghrelin mRNA found in the stomach and intestine $(11,19,20$, 26).

Peripherally, increases in circulating acylated ghrelin, but not desacyl ghrelin, has been correlated with rises in GOAT mRNA in the stomach in both fasted and dietinduced obese mice (137). The main source of circulating peripheral ghrelin is the stomach (13). Both growth-hormone-releasing hormone (GHRH) and leptin have been found to increase GOAT mRNA in cell cultures derived from mouse pituitary tissue, while somatostatin has been found to decrease GOAT mRNA (137).

Sakata and colleagues (26) have reported that $14.5 \%$ of gastric mucosal cells and $19.4 \%$ of duodenal cells that express GOAT mRNA do not show co-expression with ghrelin based on immunoreactivity. Furthermore, $28.8 \%$ of ghrelin-immunoreactive gastric cells do not co-express GOAT mRNA. While these high percentages of cells not co-expressing both proteins may be due to incomplete labeling, examining the consequences of these results may help in devising new and novel experiments. Several hypotheses can be deduced from Sakata et al.'s data. First, the high level of overlap in GOAT and ghrelin mRNA expression support the idea that acylation by GOAT occurs within the same cell that ghrelin synthesis occurs, which has been suggested by others who hypothesize that acylation occurs at the proghrelin stage of posttranslational processing $(20,128,140)$. Second, the lack of GOAT mRNA in a considerable number of both gastric mucosal and duodenal cells suggest that either some cells cannot produce acylated ghrelin, instead only produce desacyl ghrelin (along with obestatin, which is discussed later), or that acylation may also occur in a paracrine manner. If such a paracrine model were accepted, one would have to conclude that desacyl ghrelin can cross the cell membrane to gain access to GOAT in neighboring GOAT-expressing cells, once secreted by the synthesizing cell, although this seems unlikely. Furthermore, if such cells do indeed produce desacyl ghrelin, it would add further to the 
already abundant evidence that desacyl ghrelin has a physiological role. Sakata and colleagues (26) point out that the expression of GOAT mRNA in some cells that do not express ghrelin mRNA suggest the presence of another target that GOAT is capable of acylating, other than ghrelin. Therefore, while our current knowledge only points to one target of GOAT, it is important to consider that GOAT-inhibiting drugs may have unidentified effects on other pathways, which would naturally lend to more side-effects.

\subsection{Posttranslational processing of the ghrelin gene product produces several peptide hormones}

Prior to acylation by GOAT, immediately after translation, ghrelin exists as a 117 amino acid prepropeptide that undergoes a series of posttranslational modifications leading to the formation of mature ghrelin (see Figure 1.3). Preproghrelin's N-terminal 23 amino acid signal peptide is cleaved to form proghrelin by signal peptide peptidase, with the processed proghrelin being released into the ER lumen. GOAT enzyme embedded in the ER membrane then acylates proghrelin $(20,128,140)$. The intralumenal location of ghrelin during acylation suggests that GOAT's active site is also intra-lumenal. Subsequently, the $66 \mathrm{C}$-terminal amino acids are cleaved by prohormone convertase $1 / 3\left(\mathrm{PC}_{1 / 3}\right)$, likely in secretory granules or in the trans-Golgi (140142). It is important to point out that $\mathrm{PC}_{1 / 3}$ is not specific to ghrelin processing, as it has also been found to process other peptides such as glucagon-like peptide-1 (143) and cholecystokinin (144), and therefore inhibiting $\mathrm{PC}_{1 / 3}$ would have pleiotropic effects. While it is believed that in vivo GOAT acylates proghrelin, in in vitro systems acylation has also been shown to occur with much smaller peptide fragments. Due to the intracellular, ER-bound nature of GOAT, a GOAT inhibitor must be able to pass through the cellular membrane for it to be effective.

The 66 amino acid C-terminal fragment cleaved from proghrelin can be further processed into another peptide, obestatin (145). While initially obestatin was identified as an anorectic agent that binds to GPR39, with opposite effects as ghrelin (145), these data remain highly controversial as suggested by several studies (146150). While the role of obestatin has not yet been identified, it is important to consider that ghrelin knockout animals also lack obestatin, and that while GOAT inhibition may reduce acylated ghrelin levels, it might not have any direct effects on obestatin, provided that $\mathrm{PC}_{1 / 3}$ can still cleave the unacylated form of proghrelin. One would expect, however, that transcriptional or translational inhibition on the expression of the ghrelin gene, perhaps through a negative feedback mechanism that may involve ghrelin, would also inhibit obestatin synthesis.

Interestingly, a ghrelin splice variant, In2-ghrelin, that contains intron 2 of the 


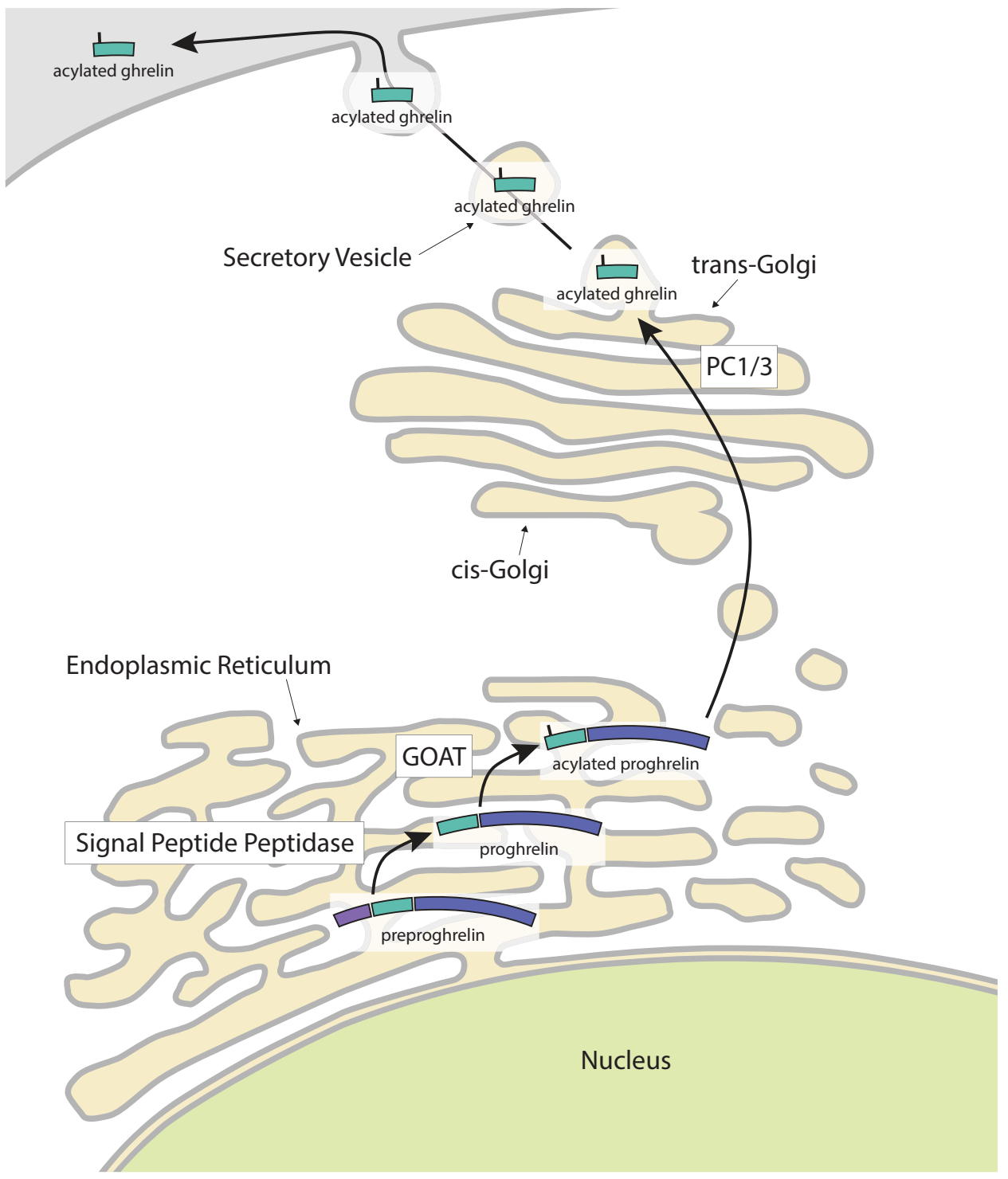

Figure 1.3: Acylated ghrelin synthesis and secretion.

Ghrelin synthesis begins with translation of ghrelin mRNA to the 117 amino acid preproghrelin. The signal peptide is cleaved by signal peptide peptidase to produce proghrelin within the ER lumen. GOAT, embedded in the ER membrane, acylates proghrelin, which transitions through the Golgi apparatus. In the trans-Golgi or within secretory vesicles, the $\mathrm{C}$-terminal 66 amino acids are cleaved resulting in mature acylated ghrelin, which subsequently is secreted from the cell. 
ghrelin gene, has been identified $(151,152)$. While mRNA for this variant has not been found in stomach extracts, it has been found to have levels 10-fold higher than native ghrelin in the pituitary and 50-fold higher in the hypothalamus (151). Reflecting a similar regulation to native ghrelin, In2-ghrelin levels rise in the pituitary following a 24-hour fast, with reduced amounts in both the pituitary and hypothalamus in diet-induced obese mice (151). Kineman and colleagues (151) suggest that this response of In2-ghrelin points to a role in metabolic stress. While direct examination has not been conducted, it is possible that In2-ghrelin can also be acylated by GOAT, due to the presence of the GSSFL N-terminal recognition core (discussed in Section 1.9). Additionally, this splice variant does not contain the coding region for obestatin (151), and therefore In2-ghrelin synthesis does not lead to increases in obestatin levels. Furthermore, whether In2-ghrelin can bind to GHSR1a is not known. In addition to this In 2 variant, an In 1 variant has also been identified with mRNA levels that closely parallel those of GOAT mRNA in various human tissues (137). This paralleled pattern of expression between In1-ghrelin and GOAT transcripts suggest that the In1-ghrelin variant may be an acylation target for GOAT, and therefore may also be affected by GOAT inhibition. It is therefore important to understand the roles of ghrelin splice variants, as GOAT inhibition would likely affect these variants as well. At the moment, however, little is known regarding In2- and In1-ghrelin.

\subsection{GOAT acyl substrates are derived from the diet}

GOAT's source of fatty acid-CoAs required for acylation has been shown to originate primarily from ingestion of medium-chain fatty acids and medium-chain triglycerides $(153,154)$. Being the main synthesis area of ghrelin, the stomach is at a suitable location to provide fatty acid substrates for acylation. By feeding mice a diet rich in certain free fatty acids, such as n-hexanoic acid and n-decanoic acid, or certain triacylglycerols, such as glyceryl trihexanoate and glyceryl tridecanoate, Nishi et al. (153) were able to manipulate the proportion of circulating acylated ghrelin containing a side-chain of a specific length, corresponding to that of the fatty acid lengths in the diet. Using this method, Nishi and colleagues demonstrated that ghrelin acylation requires medium chain fatty acids, but shorter chain (eg. C4) or longer chain fatty acids (eg. C16) cannot be used as acylation substrates (153), even though Heppner et al. demonstrated that synthetically made C16-ghrelin can still activate GHSR1a (155). While mammalian cells cannot synthesize heptanoic acid, and under normal conditions $\mathrm{n}$-heptanoyl ghrelin is not present in vivo, when mice were given a diet rich in n-heptanoic acid or glyceryl triheptanoate, n-heptanoyl ghrelin was observed in the stomach, providing strong evidence that the ghrelin side-chain originates from the diet (153). Despite this, the possibility of de novo synthesis of fatty acids still remains 


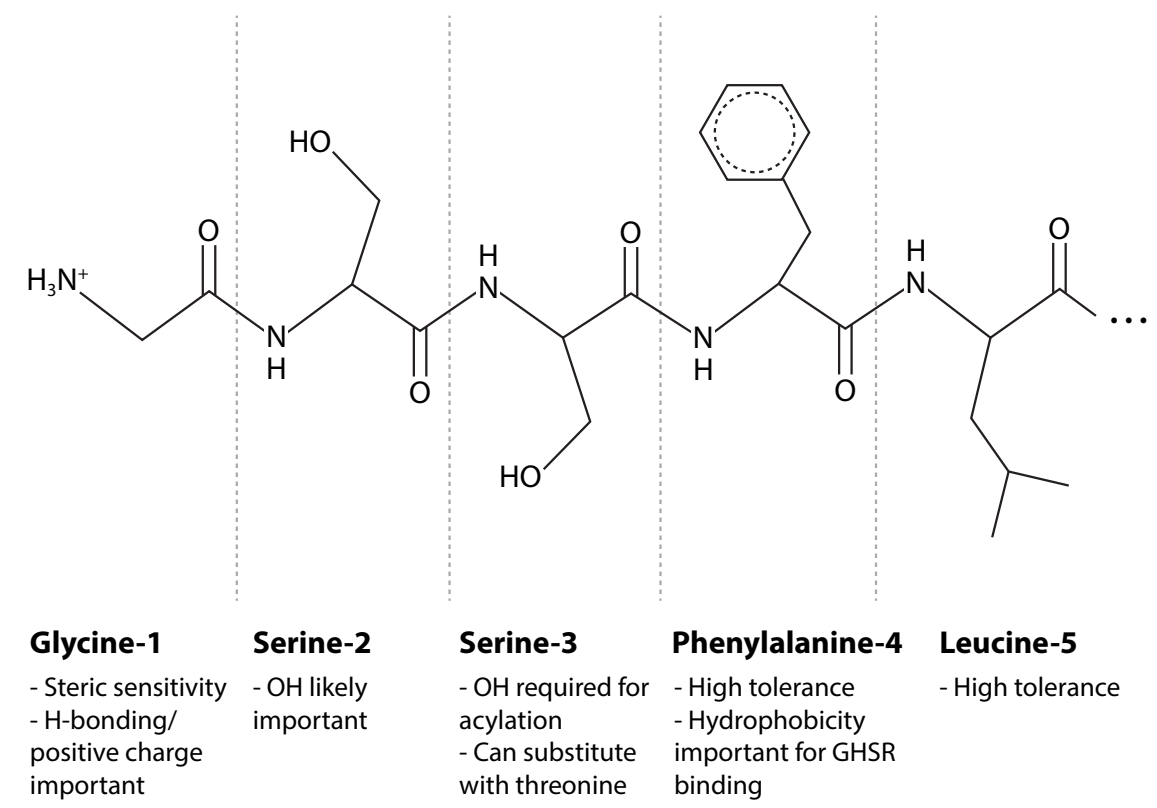

Figure 1.4: Amino acid properties important for GOAT recognition.

Selected properties of the first five amino acids of ghrelin important for recognition by ghrelin O-acyltransferase.

a possible additional source.

\subsection{The active core of ghrelin for receptor and GOAT recognition consists of the first four and five amino acids, respectively}

Interestingly, ghrelin, whether acylated or not, has no well-defined secondary or tertiary structure, suggesting that recognition by GHSR1a is sequence-based, or that only upon binding, a more stable conformation with a well-defined secondary structure is obtained $(156,157)$. Indeed, the short peptide sequence GSS(n-octanoyl)F, consisting of the first four amino acids of ghrelin along with its acyl side-chain, is sufficient to bind to and activate GHSR1a (158). Such a short peptide sequence is very likely unstructured in solution. These data might also suggest that recognition of unacylated proghrelin by GOAT during the acylation process is also sequence based. In addition 
to the short sequence required for GHSR1a activation, peptides consisting only of the first five amino acids can be acylated by GOAT $(159,160)$. In this section, details on the critical N-terminal amino acids of ghrelin will be discussed.

\subsubsection{Residue 1: Glycine}

Increasing the steric bulk of the N-terminal glycine by adding two or more methyl groups successively decreases hGOAT activity (160). Removal of the $\mathrm{N}$-terminal amine eliminates hGOAT activity (160) and it is suggested that the ability of the N-terminus to donate a hydrogen bond or provide a positive charge is required for GOAT to recognize ghrelin (161). Yang et al. (159) have also shown that substituting glycine-1 with serine significantly reduces mouse GOAT activity.

\subsubsection{Residue 2: Serine}

In a fluorescent GSSFLC $\mathrm{AcDan}_{\mathrm{n}}$ peptide fragment, recognition at the second residue by hGOAT has been suggested to be highly dependent on the presence of a hydroxyl group. Replacing the hydroxyl group at this position with a hydrogen by substituting in alanine leads to a significant reduction in reactivity, while addition of a methyl group by substituting in threonine has little effect $(160)$. Removing the hydroxyl group of the substituted threonine (in this case replacing it with a methyl group by substituting in valine) reduces reactivity significantly (160). Despite this, Yang et al. (159) have shown that replacement of serine- 2 in proghrelin with alanine has no effect on mouse ghrelin O-acyltransferase (mGOAT) activity. Darling et al. (160) suggest that this discrepancy may be a result of intrinsic differences between hGOAT and mGOAT, or due to the use of proghrelin by Yang and colleagues, rather than the GSSFLC AcDan $_{1}$ peptide fragment as used by Darling and colleagues.

\subsubsection{Residue 3: Serine}

The third amino acid in ghrelin is the target of acylation; GOAT attaches a fatty acid at this position by catalyzing the esterification of an acyl-CoA onto the hydroxyl sidechain of serine. As such, this residue is the most important amino acid for GOAT activity. In bullfrogs, this residue is replaced with threonine, an amino acid similar to serine in that it contains a carbon-hydroxyl sidechain, but with the addition of a methyl group attached to the carbon. It has been demonstrated that both human and mouse GOAT can acylate ghrelin peptide and peptide fragments with a threonine in place of serine-3 $(20,161)$, albeit with slightly reduced reactivity.

Naturally occurring esterases act to remove the acyl side-chain of acylated ghrelin. As will be discussed, many GOAT inhibiting drugs take advantage of the importance 
of the serine-3 residue by replacing it with a synthetic residue attached via a nonhydrolyzable bond to a fatty side-chain, such as through an amide or triazole linkage. Several pentapeptide GOAT inhibitors, originally identified by Yang et al. (159), as well as the drug CF801, have an alanine in place of serine-3, which provides a template similar to desacyl ghrelin which can not be acylated due to the lack of a hydroxyl sidechain.

\subsubsection{Residue 4: Phenylalanine}

Surprisingly, a wide range of substitutions at residue 4 of the ghrelin pentapeptide has been shown to have considerable, yet reduced, reactivity with hGOAT (161). In order of decreasing reactivity, substitutions with threonine, tryptophan, alanine, tyrosine, glutamine, histidine, and valine each show residual reactivity, while substitution with glutamate and lysine both eliminate hGOAT reactivity (161). Darling and colleagues (161) suggest that this high degree of tolerance at position 4 provides a desirable means of attaching additional compounds or unnatural amino acids to ghrelin mimetic GOAT inhibitors. Yang et al. (159), however, have shown that a phenylalanine-4 to alanine substitution results in considerably less, but still present, reactivity, and suggest that phenylalanine- 4 is an important component for mouse GOAT recognition. In addition to these GOAT-activity results, as will be discussed below, phenylalanine4 appears important for embedding of acyl-ghrelin peptides into plasma membranes, which is important for binding and activation of GHSR1a. So while GOAT is fairly tolerant to changes at Phe- 4 , binding to membrane-bound GHSR1a is considerably less so.

\subsubsection{Residue 5 and beyond}

Substitution of residue 5 (leucine) or residue 6 (serine) with alanine results in only minimal reduction in mouse GOAT reactivity, while substitution of residue 7 (proline) with alanine has no effect (159). Little research has been conducted on the requirements of residues beyond leucine- 5 . This in part stems from findings that only the first five amino acids are required for GOAT acylation and GHSR1a binding, and indeed many studies utilize only the $\mathrm{N}$-terminal pentapeptide of ghrelin. Due to fairly high tolerance at these residues, as is the case with phenylalanine- 4 , the sequence beyond residue 5 may provide a suitable location for peptide modification of GOAT inhibitors, including introduction of synthetic compounds meant to stabilize the compound. GOAT inhibitors such as GO-CoA-Tat that act as non-hydrolyzable acyl ghrelin analogs generally incorporate at a minimum the first five amino acids of ghrelin. While the focus of these structure-function studies has been recognition by GOAT and GHSR1a, the active core of desacyl ghrelin is much less understood. Recent development of the desacyl ghrelin analog AZP-531 suggests that the active core 
consists of residues 6 to 13 (162). It is interesting that it may be this region that provides the primary signaling component of desacyl ghrelin, as it includes amino acids 11 and 12, which differ between human and rat/mouse ghrelin. This may suggest that there are important differences in activity and recognition between human and rodent desacyl ghrelin. AZP-531 will be discussed in more detail in later sections. Identification of a yet-unknown desacyl ghrelin receptor will certainly aid in further characterizing desacyl ghrelin.

\subsection{Structure and active site of ghrelin O-acyltransferase}

As mentioned above, the core recognition sequence of ghrelin has been identified, but there is still much research to be done on the details of the enzymatic reaction and the active sites of the GOAT enzyme. Difficulties in solubilizing GOAT in its active conformation have prevented detailed structure-mechanism analysis (159). GOAT is a monomeric integral protein found embedded in the membrane of the endoplasmic reticulum (154). It belongs to the MBOAT super-family whose members are membrane-bound proteins that attach fatty acids to proteins and lipids and are involved in lipid-biosynthetic and lipid-signaling pathways (141). Members of the MBOAT family have either lysophospholid, cholesterol, diacylglycerol, or protein acyltransferase activity and represent important components of both the de novo and remodelling pathway of glycerophospholipids, embryogenesis, development, and nutrient sensing $(163,164)$. Similar to hedgehog acyltransferase (Hhat) and porcupine (164), GOAT has protein O-acyltransferase activity and it is believed that GOAT's catalytic domain is located within the ER lumen $(20,165)$, where ghrelin acylation occurs. Due to the lumenal nature of the catalytic domain, it has been suggested that in GOAT assays examining activity using microsomal fractions and exogenously applied desacyl ghrelin that at least some of the purified microsomes are in an inside-out fashion, with the once lumenal catalytic domain now extending to the exterior of the microsomes. Alternatively, it has also been suggested that GOAT acts as an acyl-CoA transporter, as suggested by the cytosolic location of acyl-CoA synthesis through $\beta$-oxidation (20).

The MBOATs show conserved polar residues within and around membranespanning regions $(141,165-170)$. Both histidine 338 and asparagine 307 in GOAT, located within the $\mathrm{C}$-terminal region, are conserved residues important for catalytic activity. His-338 is located within a short lumenal loop in a long hydrophobic region and is required for GOAT acyltransferase activity $(20,159)$. The cytosolic Asn-307 is located within a hydrophilic region and, like His-338, is required for GOAT's catalytic activity $(20,154)$. Based on hydropathy plots, Yang et al. initially 
suggested that GOAT has 8 transmembrane regions (20). Subsequently, based on results from the bioinformatics tools MemBrain (171) and MEMSAT-SVM (172), Taylor et al. (154) suggested that GOAT has 11 transmembrane spanning regions with one re-entrant loop, with a lumenal $\mathrm{N}$-terminus and a cytosolic $\mathrm{C}$-terminus. The invariant His-338 was localized to either the lumenal loop or embedded within the ER membrane near the lumen in the turn between transmembrane regions 8 and 9. The conserved Asn-307 was localized to the cytosolic region in the turn between regions 7 and 8. This topology was supported by additional data derived from phylogeny, computational prediction, epitope mapping, and induced glycosylation experiments (154). The importance of the C-terminus (of which His-338 and Asn-307 belong) in catalytic activity is further supported by results of alignment of various members of the MBOAT family, with a shared C-terminal motif found in both the lysophospholid and mouse GOAT members (163).

While His-338 is believed to be important for GOAT's catalytic activity, Taylor et al. (154) have suggested that the extra-lumenal location of Asn-307 indicates a role in substrate interactions, transport, or structural stability. GOAT acylating activity is abolished by substituting His-338 (19, 20) or Asn-307 (20) with alanine. Additionally, photocross-linking studies between GOAT and a biotinylated analog of the GOAT inhibitor GO-CoA-Tat indicate that binding occurs within a $15 \mathrm{kDa}$ fragment of the C-terminal region of GOAT (154), within which His-338 and Asn-307 are located. These data suggest that selecting certain types of drugs from a larger library (such as an aptamer library) that successfully inhibit GOAT may preferentially target the areas surrounding these two residues. As discussed in section 1.9, various mutation studies have been conducted to probe the structure and selectivity of the GOAT active site.

\subsection{GHSR1a has high levels of constitutive activity}

As discussed in section 1.4, when ghrelin binds to the GHSR1a, $\mathrm{Ca}^{2+}$ is mobilized from intracellular stores through a $\mathrm{G} \alpha_{\mathrm{q}}$-coupled PLC pathway. In the absence of ghrelin binding, GHSR1a continues to signal through the same pathway at a magnitude of approximately $50 \%$ of the maximal ligand-bound signaling capacity (173, 174). While constitutive activity is not an unusual phenomenon among G-protein coupled receptors (GPCRs), GHSR1a's level of constitutive activity is unusually high, suggesting that this basal activity likely has an important physiological role. This also makes examination of inverse agonists for GHSR1a especially important, and the first study identifying GHSR1a constitutive activity also identified that [D-Arg ${ }^{1}$, D-Phe ${ }^{5}$, D-Trp ${ }^{7,9}$, Leu $^{11}$-substance $P$, which up to that point had been identified as a synthetic GHSR1a antagonist, is in fact an inverse agonist of the receptor (173). This high constitutive activity also suggests that GHSR1a may represent an important receptor for 
the study of GPCR constitutive activity in general. Through greater examination of the mechanisms of this basal signaling, the study of GHSR1a may provide insight into how GPCRs function in the absence of a ligand.

The importance of GHSR1a constitutive activity is demonstrated by a mutation found in humans in which constitutive activity is lost, while leaving ligand-bound signaling intact. The two unrelated families identified as carrying this mutation have familial short stature (175). This missense mutation, A204E, involves a change in polarity and charge, and is located within an invariant stretch of GHSR1a. It seems reasonable to suspect that reductions in basal GHSR1a-dependent GH secretion are at least partially responsible for the short stature in these individuals. Interestingly, reductions in constitutive activity in GHSR1a-A204E are also associated with a 20\% drop in cell-surface expression of the receptor, which further contributes to reduced GHSR1a signaling (175). The origin of this reduced surface expression is not known. While one may speculate that a natural inverse agonist for the GHSR1a may exist, regulation of constitutive activity may also be delegated to GHSR1a dimerization partners, which can affect intracellular signaling. How GHSR1a constitutive activity is affected in obesity has not been examined, but remains an intriguing question to be answered. Indeed, if the ghrelin desensitization found in obesity (discussed in section 1.16) occurs at the receptor level, one may suspect that constitutive activity is also affected.

Holst and colleagues (173) initially suggested that one possible mechanism of GHSR1a constitutive activity may be due to a salt-bridge between Arg-281 (ArgVI:20) and Glu-124 (GluIII:09), which would act to bring transmembrane regions TM-VI and TM-III closer together. During typical ligand binding of a GPCR, extracellular segments of the receptor move inward, particularly transmembrane regions TM-III, TM-VI, and TM-VII, with a corresponding outward movement of the intracellular segments, pivoting around proline residues embedded within the lipid bilayer (176179). This outward movement is believed to expose epitopes on the receptor that can then be recognized by intracellular signallers. A salt-bridge between Arg-281 and Glu124 may thus simulate weak binding of a ligand, pulling extracellular segments closer together and exposing intracellular segments to various signallers, including $\mathrm{G} \alpha_{\mathrm{q}}$. Mutational analysis further identified the aromatic PheVI:16 as an important "molecular switch," with mutations at this position allowing the modulation of constitutive activity (174). This residue belongs to a group of aromatic amino acids on the inner faces of transmembrane regions TM-VI and TM-VII that appear important for constitutive activity (174). Aromatic residues (phenylalanine, tyrosine, and tryptophan) on extracellular segments may also be important for ligand binding by contributing cation- $\pi$ interactions, which are strong non-covalent electrostatic interactions, in this case involving the benzene rings of the amino acids' sidechain (180). In addition to PheVI:16, both PheVII:06 and PheVII:09 appear important for GHSR1a's constitutive activity (174). These three residues are proposed to constitute part of a hydrophobic core that 
brings the extracellular ends of TMs VI and VII closer together (174).

In addition to its constitutive activity, GHSR1a is also to a large extent constitutively internalized in the absence of a ligand, with the majority of the receptors appearing to be associated with endosomes (174). Endosomes can later localize with lysosomes for degradation or returned to the plasma membrane for reuse (181). The fraction of internalized GHSR1a should affect the overall magnitude of intracellular signaling provided by constitutive activity, in addition to the cell's normal response to ghrelin. Internalization can be attenuated with an inverse agonist (174). These observations are important, as they suggest that increases in GHSR1a expression may not always correspond to an increased response to ghrelin. The mechanisms by which internalization and surface expression are modulated remains to be examined, and identification of a natural GHSR1a inverse agonist may shed light on these mechanisms.

\subsection{GHSR1a signaling potency and timeframe depend on the length of ghrelin's fatty-acyl side chain}

While acylation of ghrelin often involves a C8 octanoyl fatty acid, as discussed fatty acids of other lengths can also act as acylation substrates. Interestingly, the length of the side chain affects ghrelin's timeframe and potency of action on GHSR1a. In COS7 cells transiently expressing GHSR1a, C2-ghrelin shows no GHSR1a-dependent response, while C6-, C8-, and C16-ghrelin show very similar responses (155). Nishi and colleagues (153) have also demonstrated similar time-courses in $\left[\mathrm{Ca}^{2+}\right]_{\mathrm{i}}$ signaling for C6-, C7-, and C8-ghrelin in CHO-GHSR62 cells, with C8-ghrelin showing the highest potency. In vivo data indicate that $\mathrm{C} 16$-ghrelin shows a delayed effect when given acutely ICV, with no changes in cumulative food intake four hours post-injection but significantly elevated cumulative intake 24 hours after injection. C8-ghrelin, on the other hand, does increase cumulative food intake at the shorter four-hour timepoint but returns to vehicle levels after 24 hours (155). The acute single injection might explain this regression to control levels. In contrast, when given chronically ICV over six days, C6-, C8-, C12-, C14-, and C16-ghrelin all induce increases in fat mass to varying degrees, while C2-ghrelin fails to show an effect (155). The gain in fat mass when given peripherally over six days is greater in C16-treated vs. C8-treated animals (155).

These data suggest that the type of fats consumed may induce different physiological responses, including modulation of intensity and timing of hunger and satiety signals. This adds further to the notion that the type of fats consumed may be just as important as the amount of fats consumed when dieting. Furthermore, a GOAT inhibitor may display variation in its ability to inhibit acylation with different length fatty 
acids, which may be based on such parameters as the energetic stability of the acylated ghrelin end-product or intermediary structures, or the ability of the desacyl ghrelin and fatty acyl CoA substrates to associate. These data also suggest that for treatments where acylated ghrelin administration would be beneficial, such as in treatment for cachexia, the length of the side chain is a parameter that should be optimized. Indeed, based on Heppner and colleagues' (155) data, a C16 side chain, which displays more prolonged and enhanced potency of effects on body weight and adiposity, may be beneficial for such treatments. Heppner et al. suggest that C16-ghrelin's prolonged and enhanced effects may be due to the long side-chain's increased ability to be embedded in the hydrophobic plasma membrane, which would decrease clearance and likely also delay the response (155). This embedding appears to be dependent on the fatty acyl side chain as well as ghrelin's aromatic phenylalanine- 4 residue, which as discussed is a required amino acid for GHSR1a binding (156). A short C2 side-chain may be too small to allow such embedding (155). Regardless, the majority of circulating acylated ghrelin have C8 or C10 side chains (19).

\subsection{Transport of ghrelin across the blood-brain barrier}

There is considerable debate as to whether or not ghrelin can cross the blood-brain barrier. Much of the data looking at this question have been supplied by Banks and colleagues (see (182) and (183)), who have also examined leptin's ability to cross the blood-brain barrier. Given that GHSR1a is expressed in the arcuate nucleus, and that this hypothalamic area is in close proximity to the median eminence-a circumventricular organ with a weak blood-brain barrier-the possibility of peripheral ghrelin accessing the arcuate nucleus seems plausible.

Interestingly, in normal-weight healthy mice, differences in transport have been demonstrated between exogenous human and mouse ghrelin. While human ghrelin administered to mice is transported in both the blood-to-brain and brain-to-blood directions by a saturable system, mouse ghrelin is transported from brain-to-blood but is much more limited in the opposite direction (182). Whether this difference occurs in humans as well is not known. If it does, then animal research, particularly in mice, on the central effects of peripherally synthesized or administered ghrelin may not be fully translatable to humans. This does, however, raise the possibility of administering mouse ghrelin for human treatment if one desires to limit access to the brain, which may aid in reducing side-effects. Unacylated mouse ghrelin, on the other hand, travels from blood-to-brain by nonsaturable transmembrane diffusion, which quickly becomes sequestered and retained within the brain (182).

Obesity and other physiological states can affect the blood-brain barrier. Leptin, which is secreted by adipose cells and has an inverse relationship with ghrelin, has been 
shown to cross the blood-brain barrier, and the ease by which this occurs depends on metabolic status. In particular, obesity is associated with impaired transport of leptin across the blood-brain barrier, a phenomenon contributing to obesity-associated leptin resistance (184-186). Additionally, obese mice are also impaired in their ability to transport ghrelin from the blood into the brain (183). The opposite is seen in fasted animals (183). Overall, a negative relationship exists between body weight and brain uptake of acylated ghrelin. Interestingly, this reduction in transport of both leptin and ghrelin with obesity goes beyond these two metabolic signalers. Ouyang et al. (187) have shown that diet-induced obese mice have reduced energy metabolism in cerebral microvessels of the blood-brain barrier, associated with downregulation of a wide range of proteins involved in, among others, cell structure, enzymatic activity, protein transport, and stability. This reduced energy metabolism results in reduced transport of various factors across the blood-brain barrier, so that while the orexigenic signal derived from peripheral ghrelin may be reduced, there are also reductions in anorectic signaling such as through reduced leptin uptake. How these changes manifest themselves behaviorally and physiologically is not fully understood.

A further question that arises is whether or not the brain-to-blood transport of acylated ghrelin has a physiological role on peripheral signaling. Once ghrelin reaches the periphery's circulation, does it become diluted enough relative to stomach-derived and other peripherally-derived ghrelin to not have any significant effect? This was addressed in part by Theander-Carrillo et al. (67), who chronically (over 6 days) administered a low dose of acylated ghrelin peripherally, similar to a dose given intracerebroventricularly that was found to have various effects on metabolism, and found no changes in body weight, energy balance, or lipid and glucose metabolism. This did not address, however, the possibility of a greater concentration reaching the pituitary from the hypothalamus. In the other direction, the considerable increase in circulating ghrelin during fasting or food restriction may affect transport rates into the brain. This has been demonstrated previously and the starvation-induced rise in circulating triglycerides have been suggested to be one of the factors that promote uptake of ghrelin (183). The role of diffusion of unacylated ghrelin into the brain is also unknown. It is unlikely that this ghrelin can be acylated, particularly due to GOAT's cellular location, but part of its sequestration once inside the brain may involve binding to an unidentified desacyl ghrelin receptor.

\subsection{Leptin has inhibitory effects on the ghrelin system}

Like ghrelin, the initial identification of leptin spurred a slew of experiments looking at its role in obesity. Leptin is an anorectic signaller that inhibits NPY/AgRP and promotes CART through the leptin receptor (188-193). This hormone is released by 
adipocytes to provide a gauge of energy supply in the form of fats. Released into circulation, leptin crosses the blood-brain barrier to bind to the leptin receptor to enhance satiety signaling. One promising study demonstrated that low doses of daily leptin injections to individuals who have lost weight can alter metabolism in ways that may aid in avoiding weight regain, specifically by increasing energy expenditure and thyroid hormones (194). The same group has also demonstrated that leptin injections can reverse the increases in neural activity associated with visual food cues in food-related regulatory and hedonic circuits (195). Maintenance of weight after weight loss is of primary importance to individuals who have successfully reduced their body weight, as this can be more difficult than the weight loss itself. During this period, several factors promote weight regain, including decreased energy expenditure (194, 196-198) and sympathetic nervous system tone (199-202), decreased levels of leptin, thyroxine, and triiodothyronine $(194,200)$, as well as increased hedonic neural responses to visual food stimuli (195). Low doses of daily leptin injections help reverse these changes and hence aid in eliminating some sources contributing to weight regain $(195,203)$. On the other hand, obese individuals who have not lost weight show increased resistance to leptin associated with elevated leptin levels (204-212) as well as impaired transport of leptin across the blood-brain barrier $(212,213)$, often making leptin administration ineffective.

Data indicate that leptin has negative control on GHSR1a expression in the arcuate nucleus. Chronic leptin treatment is required to maintain reduced GHSR1a levels, as a single ICV injection of leptin only shows a non-significant decrease in GHSR1a mRNA two hours after injection, whereas 7 days of ICV leptin infusion results in significantly reduced GHSR1a mRNA in the arcuate of both ad libitum-fed and 48 hour fasted rats (119). Fasting-induced increases in GHSR1a mRNA can also be reduced by ICV leptin (119). Additionally, Zucker fatty rats that display reduced leptin signaling have significantly elevated GHSR1a expression in the arcuate nucleus (119). These effects appear to be opposite to that of ghrelin, which induces increases in GHSR1a mRNA in the arcuate nucleus two hours after a single ICV injection as well as after 7 days of ICV infusion (119). A negative correlation has also been found between levels of circulating leptin and ghrelin (214).

\subsection{Development and rearrangement of hypothalamic feeding circuits}

Along with projections within the confines of the arcuate nucleus, projections extend from the arcuate to the PVN, dorsomedial nucleus of the hypothalamus (DMH), and lateral nucleus of the hypothalamus (LH) (for a review of hypothalamic neurocircuitry, see (215)). These projections to non-arcuate areas develop early on during the perinatal 
period and reach a relatively permanent state that carries on into adulthood. Leptin deficient $o b / o b$ mice show reductions in fiber density within these areas, originating from the arcuate, as early as postnatal day 10 , which can be attenuated by leptin treatment from P4 to P12 (216). This reduction in fiber density is associated with a dampening of the trophic effect of leptin (217). Ob/ob mice that do not produce leptin have greater excitatory tone onto NPY neurons and inhibitory tone onto POMC neurons (218). In contrast, circuitry confined to the arcuate display fairly rapid rearrangement in response to leptin and ghrelin. Four days of peripheral ghrelin treatment results in a decrease in excitatory inputs and an increase in inhibitory inputs onto POMC neurons, although no effect is found on NPY neurons (218). Rewiring of arcuate circuits can also be seen as early as 6 hours after a single IP injection of leptin in both wild-type and $o b / o b$ mice, reversing abnormalities in $o b / o b$ wiring to a wild-type state (218). Interestingly, leptin's trophic effects appear to be limited to arcuate projections, as $\mathrm{DMH}$ to ventromedial nucleus of the hypothalamus $(\mathrm{VMH})$ as well as limbic to hypothalamic projections are unaltered (216), although effects in other pathways can not be ruled out. The crucial period from P 4 to P12 corresponds to a developmental phase in which a surge in leptin has been reported (219). Whether a similar ghrelin surge occurs during early development is not yet known, but ghrelin's reported trophic and plasticity effects $(218,220)$ suggest that ghrelin may also provide a crucial signal for hypothalamic development.

\subsection{Obesity is associated with ghrelin resistance and hypothalamic dysregulation}

Development of obesity is associated with various metabolic and hormonal changes. Circulating ghrelin levels have been found to be significantly reduced in obese humans and rodents (221-224). In addition to this reduction, the typical preprandial rise in ghrelin levels is significantly reduced in diet-induced obese (DIO) mice, and the postprandial decrease has a slower onset (1 hour in lean mice compared to 12 hours in obese mice) (223). Furthermore, while lean mice maintained on a standard chow or low-fat diet respond to both peripherally and centrally administered ghrelin by increasing food intake, such an effect is attenuated in DIO mice (224). This desensitization to ghrelin's effect when given centrally suggests that reduced central signaling is not entirely due to reduced transport across the blood-brain barrier. Additionally, DIO mice also show desensitization to fasting-induced elevations in endogenous ghrelin, with a reduced feeding response (224). If DIO mice are subsequently switched to a low-fat diet, these animals will respond to exogenous ghrelin after only three days on the diet, consuming almost twice as much food compared to saline-treated animals in the first two hours after injection (223). After 13 days on the low-fat diet, this increases 
to almost four-fold (223). At both 3 and 13 days into the low fat diet, animals show a progressive and significant reduction in body weight to levels comparable to lean mice maintained on a low-fat diet at the 13-day timepoint (223). These data demonstrate that the diet-induced resistance to ghrelin in obesity is reversible, and that even shortterm reductions in body weight may result in an increase in ghrelin's orexigenic signal, making weight loss difficult. This suggests that the ghrelin system may in part provide a set-point for body weight, with deviations below this being met by an increase in appetite or preference for fatty foods. While the diet provided to lab animals can be systematically controlled, it is much more difficult to prevent humans from seeking out high-calorie food.

At the transcriptional level, reductions in ghrelin and GOAT mRNA as well as reductions in hypothalamic GHSR1a mRNA occur in diet-induced obesity (224). Since exogenous ghrelin administration, either centrally or peripherally, cannot induce feeding in DIO animals to the extent that it can in chow-fed animals, it is unlikely that reductions in ghrelin and GOAT mRNA are the sole culprits in ghrelin desensitization. Reductions in GHSR1a mRNA, in contrast, may play a more important role. These changes are also associated with reduced NPY and AgRP mRNA in the hypothalamus (224). Interestingly, data from Briggs et al. (224) suggest that ghrelin desensitization originates in a pathway upstream of NPY/AgRP, since ICV injection of NPY still promotes food intake, while ghrelin effects are reduced (224). In contrast, Bouret et al. (216) have found a two to four-fold reduction in AgRP fibers within the PVN that persists into adulthood in DIO-susceptible animals, which is independent of maternal or postnatal diet. This observation suggests that ghrelin insensitivity may in part occur through alterations in NPY/AgRP neurocircuitry, and that the reduced NPY and AgRP signaling found in obesity (224) is not entirely due to an insensitivity to ghrelin but more due to an underlying change in the NPY/AgRP circuitry.

Another possible mechanism of ghrelin desensitization is internalization of the GHSR1a resulting in reduced surface expression. It has been demonstrated that the GHSR1a has high levels of ligand-independent internalization, which can be reversed by inverse agonist treatment (174). These responses suggest the GHSR1a may be quickly cycled between its internalized and surface-bound states, and that there may be certain factors that shift the balance in favor of one state over the other. Indeed, localizing mature GHSR1a to the cellular membrane or to internal compartments may prove more important than actual GHSR1a expression. It is not known if excessive and prolonged ghrelinergic activity may promote prolonged internalization of the receptor, even if ghrelin is subsequently removed. While it is generally thought that internalized receptors are eventually returned to the membrane, it remains a possibility that at least some of these receptors get degraded by intracellular machinery. Perreault et al. (223) have also suggested that resistance to ghrelin may in fact originate from leptin's inhibitory effect on NPY/AgRP activity, and weight loss, which is associated with a 
reduction in leptin levels, removes this inhibition.

The desensitization to ghrelin extends beyond the feeding system, as central administration of ghrelin results in an attenuated GH response in DIO animals compared to chow-fed animals (224). Despite this, ghrelin desensitization as a result of obesity does not occur in all areas of the brain. In particular, while DIO animals display reduced Fos-immunoreactivity in the arcuate compared to chow-fed animals, a normal response to ghrelin occurs in the PVN (224).

The consequences of ghrelin resistance may be more detrimental than at first sight. Indeed, if resistance is also found for some of ghrelin's other roles, including its ability to protect against the detrimental effects of stress and anxiety, and its role in neurogenesis as well as cognition, these changes may point to potential therapeutic treatments for other disorders that may occur in tandem with obesity, as well as in aging in which some studies report a decrease in ghrelin activity $(222,225)$. In fact, given that desensitization in some systems can often occur as a consequence of overactivity, conditions in which ghrelin may be chronically elevated, such as during prolonged stress, may induce desensitization and hence loss of ghrelin's protective effects. Furthermore, since resistance to ghrelin occurs selectively in some areas (such as the arcuate) but not in others (such as in the PVN), caution must be taken when considering ghrelin-targeting treatments, since an increased dose may have little effect in one system but an amplified effect in others.

The desensitization to ghrelin may be an attempt at reducing adiposity. Along with hyperleptinemia, a state of hyperinsulinemia and hyperglycemia are also often associated with obesity. Glucose and insulin's inhibitory and anorectic activity on hypothalamic AMPK activity has been well documented $(111,226)$ and, as with leptin, may be some of the key components contributing to the reduced basal AMPK activity found in DIO animals (227). Combined, the development of resistance to insulin, leptin, as well as ghrelin in diet-induced obesity suggests wide-spread abnormalities in feeding-related circuitry. This would also suggest that drugs targeting these systems may become less effective in obese individuals, and that obesity may be more effectively treated early on before dysregulation has fully set in. Unfortunately, obesity is often left untreated until the situation has become considerably severe. 


\section{Chapter 2}

\section{Pharmacological Agents Targeting the Ghrelin System}

The benefits of targeting the ghrelin system for treatment of metabolic disorders should be quite evident from the previous discussion. It should also be quite evident that serious side-effects from such treatment is likely. In order to properly treat metabolic disorders, however, we must expand our knowledge of this system, develop various drugs, and examine the associated benefits and risks to guide development of the next generation of drugs. Current drugs, none of which have gone through regulatory approval for use in humans, include the GOAT inhibitor GO-CoA-Tat, several pentapeptide-based drugs, spiegelmers, anti-ghrelin antibodies (using both active and passive immunization), and the desacyl ghrelin analog AZP-531. Along with the potential as therapeutic agents, these compounds provide valuable information for understanding the ghrelin system. In this chapter, I will discuss the various techniques to target the ghrelin system in treatment of metabolic disorders, particulary obesity and type II diabetes.

\subsection{Different phenotypes of ghrelin and GOAT knockout animals are revealed by exposure to high-fat diet}

An examination of both ghrelin and GOAT knockout animals may allow us to predict the effects of GOAT inhibition or other drugs targeting the ghrelin system. While ghrelin knockout animals would be lacking the target of GOAT and hence would have no acylated ghrelin, these animals would also have no desacyl ghrelin as well as no obestatin, which currently has no identified role. Despite this, examination of knockout animals can still provide clues as to the physiological and behavioral consequences 
of targeting the ghrelin system, and suggest important areas of examination when designing experiments looking at drug treatment.

On a diet consisting of standard chow, ghrelin knockout mice do not differ from wild-type mice in terms of body weight and food intake $(228,229)$. Despite this, ghrelin knockout mice display a reduced respiratory quotient, particularly on a highfat diet, indicating increased fat utilization despite a lack of difference in total food consumption compared to wild-type animals $(18,228-231)$. In terms of size, growth rate, gross behavior, viability, fertility, appetite, bone density, gastric emptying, and fat deposition, ghrelin knockout mice on standard chow are indistinguishable from wildtype mice (228-230). Ghrelin knockout mice have also been found to have enhanced insulin secretion and reduced high-fat diet-induced glucose intolerance (31). Six-week old ghrelin knockout animals have also shown a resistance to high-fat diet induced obesity, with these effects lasting until at least week 24 (231). In contrast, Sun et al. (232) have reported that ghrelin knockout mice as well as ghrelin receptor knockout mice do not show any differences in body weight or energy expenditure compared to wild-type animals even while on a high-fat diet (232).

In one study, GOAT knockout mice fed standard chow showed normal body weight and fat mass, but when given ad libitum access to a diet rich in medium-chain triglycerides showed slightly increased food intake but reduced body weight owing to considerable reductions in fat mass (233). These differences can be attributed to increased oxidation of fats in the absence of acylated ghrelin. Despite this, others have shown that GOAT knockout mice do not differ in body weight compared to their wild-type litter-mates, either on a high-fat diet or standard chow (234). In these two contradictory studies, different formulations of high-fat diet were used, with possible differences in carbohydrate content.

These data suggest that differences between wild type and knockout mice may be revealed and amplified by exposure to a high-fat diet. While effects were not always found between animals on a high-fat diet, there is considerable evidence that modulation of diet is important to tease apart the role of the ghrelin system. Because of this, it is likely beneficial to study a GOAT-inhibiting drug's ability to affect metabolism and food intake while animals are exposed to a high fat diet rather than standard chow. One must also consider, however, that the ultimate goal of drug development is human treatment, so that while these highly controlled dietary manipulations may be beneficial in the lab, they must in the end translate to human conditions, in which diet is more variable with different levels of fat, carbohydrate, and protein content. 


\subsection{Measuring GOAT activity}

While at the moment there are no commercially available kits to measure GOAT activity, several labs have published the details of in-house assays, some with potential for high throughput use. Common to all assays is the collection of GOAT through baculovirus infection, in most cases using Sf9 insect cell lines, a cell line commonly used for protein expression. Due to GOAT's membrane-bound nature, purifying GOAT has shown to be difficult, and so ultracentrifuge microsomal membrane fractions are generally collected, which by nature includes other proteins and enzymes. Purification of GOAT enzyme, although in an inactive form, has been accomplished using affinity chromatography with a 3xFLAG tag appended to the enzyme's C-terminus (128).

In these assays, high levels of octanoyl-CoA hydrolysis to octanoate have been found when crude membrane fractions are included, likely due to the presence of hydrolyzing enzymes. The resulting octanoate cannot be used as a substrate by GOAT. Addition of competing palmitoyl-CoA to reaction buffers containing membrane fractions has been found to reduce hydrolysis of octanoyl-CoA from above $90 \%$ within five minutes to below $30 \%$ in the same time-frame (159). This reduction in hydrolysis has been found to be a function of the concentration of palmitoyl-CoA (159).

In an enzyme-linked click chemistry assay developed by Garner and Janda (235), biotinylated ghrelin is adhered to streptavidin-coated plates. GOAT microsomes and octynoyl-CoA (rather than octanoyl-CoA) are added to the reaction wells and allowed to incubate. Octynoyl-CoA differs from GOAT's typical octanoyl-CoA substrate by a triple bond between the two terminal carbon atoms, rather than a single bond as found in octanoyl-CoA, allowing for implementation of click chemistry. After acylation and washing, a horseradish peroxidase-azide conjugate is attached via $\mathrm{Cu}^{\mathrm{I}}$-catalyzed Huisgen $[3+2]$ cycloaddition to the terminal C-C triple bond of the n-octynoyl moiety, forming a 1,2,3 triazole complex linking the acylated ghrelin peptide to a horseradish peroxidase enzyme. A horseradish peroxidase substrate in the presence of the oxidizing agent $\mathrm{H}_{2} \mathrm{O}_{2}$ allows for colorimetric measurements of octynoylated ghrelin peptides. Beyond its use as a GOAT assay, these results demonstrate that GOAT can acylate ghrelin with side-chains containing triple bonds. The implications to in vivo systems being that GOAT may possibly recognize medium-chain unsaturated fatty acids containing double bonds. Whether such a possibility is correct, and how the resulting active ghrelin molecule would differ from traditional octanoyl ghrelin, would be an interesting question to answer.

Another GOAT assay, developed by Yang et al. (159), uses radioactive ${ }^{3} \mathrm{H}$ octanoyl-CoA, which is incubated with C-terminal His8-tagged proghrelin and GOAT microsomes. Isolation of ghrelin is accomplished through nickel-affinity chromatography, followed by liquid scintillation counting to measure ${ }^{3} \mathrm{H}$, providing a measure of acylated ghrelin levels. Yang et al. (159) observed that enzyme kinetics 
were not fully linear with the amount of GOAT included in the reaction, likely due to the presence of deacylases, palmitoyl-CoA, product inhibition, and other competing reactions. Barnett et al. (236) have developed a similar radioactive assay, using C-terminal biotinylated ghrelin allowing for mobilization with streptavidin.

In another GOAT assay, Darling et al. (160) extended the standard ghrelin(1-5) pentapeptide to include a C-terminal cysteine residue, allowing attachment of a thiolreactive acrylodan fluorophore. Following incubation with GOAT microsomes and octanoyl-CoA, the reaction solution is separated by reverse-phase high-performance liquid chromatography (HPLC) with fluorescence peak integration to measure acylated vs. desacylated ghrelin.

\subsection{Inhibition of ghrelin O-acyltransferase by the designed drug GO-CoA-Tat}

GO-CoA-Tat was initially reported in 2010 and was one of the first GOAT inhibitors showing promising in vivo effects. It has similar structural characteristics to acylated ghrelin (236). This molecule was designed to fit in the active site of the GOAT enzyme and stabilize the trimeric conformation approximating the intermediary structure involving the acyl-CoA, ghrelin, and GOAT molecules involved in the acylation reaction. In this inhibitor, a non-cleavable 8-carbon octanoyl-CoA sidechain is attached via a synthetic diamino propionic acid, which has been substituted in place of the typical serine-3 residue of the first ten amino acids of the ghrelin molecule, resulting in a non-hydrolyzable amide bond. This fragment of GO-CoA-Tat is a simple five amino acid extension to the pentapeptide inhibitor GS(Dap-octanoyl)FL initially identified by Yang et al. (159) in 2008. On the C-terminus of this fragment an amidated transactivator of transcription (Tat) peptide has been attached via an amino hexanoic acid (Ahx) linker to aid in cell penetration. Once present inside the cell, the inhibitor can then interact with GOAT enzymes embedded in the membrane of the endoplasmic reticulum.

GO-CoA-Tat has been verified as a GOAT inhibitor in cell lines, along with in vivo results consistent with current knowledge regarding acylated ghrelin and findings in GOAT and ghrelin knockout animals (236). In HeLa and HEK cell lines stably expressing GOAT and preproghrelin, GOAT inhibition occurs with an $\mathrm{IC}_{50}$ of around $5 \mu \mathrm{M}$, with maximal inhibition after 24 hours of incubation (236). In an in vitro microsomal based assay, almost complete inhibition of GOAT is observed at $100 \mathrm{nM}$ within 5 minutes (236). Barnett and colleagues suggest that this large difference in time to reach maximal inhibition may be due to intracellular acylated ghrelin stores in the cell-based assays. In an in vivo study, C57BL/6J mice treated with $40 \mathrm{mg} / \mathrm{kg}$ intraperitoneal injections of GO-CoA-Tat on a diet high in medium chain triglycerides 
showed maximal inhibition of serum acyl ghrelin levels 6 hours after administration with no changes in desacyl ghrelin levels, compared to control animals given D4-Tat (236). After one month of daily injections, treated animals displayed no differences in food intake, but reduced fat mass without changes in lean mass, resulting in an overall reduction in body mass (236). Animals receiving intraperitoneal glucose challenge along with GO-CoA-Tat treatment showed improved insulin response and reduced blood glucose compared to control animals (236). These data suggest a possible therapeutic utility of GO-CoA-Tat in humans for treatment of obesity and type II diabetes.

\subsection{Inhibition of ghrelin O-acyltransferase with designed drugs incorporating a triazole-linked lipid group}

One of the key features of Go-CoA-Tat is the non-hydrolyzable amide bond attaching the octanoyl side-chain to the ghrelin peptide. By using a stable bond, Go-CoA-Tat's half-life and hence bioavailability is increased considerably. There are, however, other covalent bonds that can be used. Zhao and colleagues (237) have developed several ghrelin mimetic inhibitors in which the lipid side chain at the third residue is attached via a triazole linkage. This linkage increases the in vivo half-life relative to drugs incorporating a more hydrolyzable ester or even amide bond. Like GO-CoA-Tat, this inhibitor is based on the GS(Dap-octanoyl)FL pentapeptide. By employing click chemistry techniques, an azide group is constructed on the Dap3 residue and attachment of alkynes is accomplished through copper-catalyzed Huisgen-1,3-dipolar cycloaddition. Furthermore, Zhao et al. (237) have demonstrated that aromatic phenyl groups attached to the distal portion of the lipid sidechain retain human GOAT inhibitory activity, with maximal inhibition with a 3-carbon phenylpropyl triazole group and a reported $\mathrm{IC}_{50}$ of $0.7 \mu \mathrm{M}$. As discussed previously, the same group of researchers had also previously reported that the fourth residue of ghrelin has large tolerance for recognition by GOAT (161), allowing addition of various side-chains at this residue, including aromatic moieties. In summary, this group's inhibitors are a group of compounds consisting of a GS(Dap)FL pentapeptide with a phenyl group attached at the F4 position and variably-lengthed alkyl-phenyl groups attached at the Dap3 position (161). These synthetic modifications together provide more stability to the compound and allow for maximal inhibitory potency. 


\subsection{Treating metabolic disorders with the desacyl ghrelin analog AZP-531}

As discussed in section 1.3, administration of desacyl ghrelin can provide beneficial effects under the correct conditions. In particular, one can see improvements in feeding behavior as well as glucose control and insulin sensitivity. Two of the most direct methods of increasing desacyl ghrelin activity are through administration of desacyl ghrelin itself or through administration of a desacyl ghrelin analog. A third possibility includes inhibition of GOAT. Interestingly, however, inhibiting GOAT does not necessarily increase desacyl ghrelin levels, which appears counterintuitive. Indeed, intraperitoneal GO-CoA-Tat reduces circulating acylated ghrelin but has no effect on desacyl ghrelin levels six hours post-injection (236). This may reflect alterations in secretion patterns, modulation of translation and/or transcription, or perhaps a feedback mechanism. Despite this, the possibility remains that more long-term treatment may result in increases in desacyl ghrelin levels.

A recently developed pharmacological compound that fits into the category of desacyl ghrelin analogs is AZP-531, a fully cyclized peptide drug containing what is claimed to be the important recognition sequence for desacyl ghrelin, amino acids 6 to 13 of the peptide (162). This sequence was identified by examining the properties of various fragments of desacyl ghrelin and their ability to induce anti-diabetogenic effects. Along with its pharmacological effects, AZP-531 and other desacyl ghrelin analogs may help in identifying a possible desacyl ghrelin receptor. If such a cellsurface receptor exists, an analog such as AZP-531 would not be required to cross cell membranes, in contrast to GOAT inhibiting drug such as Go-CoA-Tat.

Not only does AZP-531 exhibit remarkable stability in vivo and in human blood samples, its ability to behave as a desacyl ghrelin analog is convincing. In a study by Delhanty et al. (162), both desacyl ghrelin and AZP-531 treatment prevented weight gain in mice fed a high-fat diet for one week, without a change in caloric intake (162). No effects were found in animals fed regular chow. This weight loss was attributed to reductions in fat mass. Using both insulin and glucose tolerance tests on mice fed a high-fat diet for two to four weeks demonstrated that both desacyl ghrelin and AZP-531 improved glucose tolerance and insulin sensitivity to levels similar to that of animals on regular chow (162). At the time of this writing, AZP-531 is undergoing

early clinical trials for treatment of metabolic disorders, particularly type 2 diabetes and the less common Prader-Willi Syndrome. 


\subsection{Neutralizing acyl-ghrelin with antibodies}

Several groups have examined the in vivo effects of anti-ghrelin antibodies. Passive immunization involves administration of exogenous antibodies, whereas active immunization involves stimulating the body's immune response to create its own antibodies. As such, passive immunization is much more short term and requires continued administration to maintain antibody levels. The half-life for most IgG monoclonal antibodies have been reported to be 6 to 8 days in mice (238) and 22 to 23 days in humans (239). While active immunization often requires only a few injections and can potentially last a lifetime, it is also much less reversible. Mayarov et al. (240) provide four advantages of an immunotherapeutic approach to ghrelin neutralization, over other techniques such as small molecule inhibitors and receptor antagonists:

1. Immunoneutralization, when administered to the periphery, is limited to the periphery, potentially reducing side effects.

2. Safety and efficacy is highly predictable and reproducible, due to neutralization consistently occurring within the bloodstream.

3. Targeting the ghrelin ligand, as opposed to the ghrelin receptor, reduces the potential for binding to unknown targets via non-specific binding, including to potential unidentified ghrelin receptor types.

4. Antibodies have a long half-life in vivo.

While reports of vaccination have shown beneficial effects in terms of obesity and/or diabetic parameters, not all data are consistent between studies. There are several explanations for these inconsistencies, including differences in species, feeding regimen, starting metabolic status, injection timing and quantity, as well as differences in the use of monoclonal vs polyclonal antibodies with varying binding capacities and specificities. Furthermore, the structure of the ghrelin hapten and the carrier and adjuvant used to induce an immune response can contribute to differences. The current literature does, however, suggest that vaccination is a feasible route to combat obesity and diabetes in humans. In this section, the various studies examining anti-ghrelin antibodies, either produced by active or passive immunization, will be discussed and compared.

\subsubsection{Passive immunization against acylated ghrelin}

Passive immunization can involve the use of either monoclonal or polyclonal antibodies administered in various different ways. As will be discussed, the route of administration as well as the type and source of antibody determine the efficacy. Providing basic 
validation of the potential for anti-ghrelin antibodies, Lu et al. (241) demonstrated that monoclonal pretreatment can completely abolish short-term ( 2 hour) feeding induced by intraperitoneal ghrelin. In neutralizing endogenous sources of ghrelin, however, this same antibody given intraperitoneally had no effect on refeeding after a 12 hour fast (241). In contrast, Nakazato et al. (64) demonstrated that ICV administration of a polyclonal IgG can dose-dependently reduce refeeding, as well as reduce darkphase food intake by $36 \%$ in free-feeding rats. There are various explanations for these differences, including the use of monoclonal vs. polyclonal antibodies and especially central vs. peripheral administration.

In order to examine the lack of effects caused by the monoclonal antibody when given peripherally, Zakhari and colleagues (242) administrated a combination of three monoclonal antibodies, each raised against a different segment of the acylated ghrelin peptide. These antibodies were raised against acylated ghrelin(1-10), ghrelin(13-28) and the full length acylated form of ghrelin. Only the former antibody specifically bound to acylated ghrelin, while the two latter antibodies bound to both forms of ghrelin. By administering all three antibodies together, an increase in heat dissipation and $\mathrm{O}_{2}$ consumption as well as a decrease in refeeding were observed following a fast (242). Any combination of two of these antibodies, however, failed to reduce food intake, although changes in energy expenditure and carbohydrate utilization were still present. The ability of the triplet combination of monoclonal antibodies to effectively reduce food intake has been suggested to be as a result of affecting the secondary structure of various amphiphilic segments of acylated ghrelin (242). By not only masking the signalling serine- 3 component of the peptide but also sequences more C-terminally located, a combination of multiple monoclonal antibodies is considerably more effective at preventing activation of GHSR1a.

Zakhari et al. (242) suggest that while doublet-binding of antibodies to the peptide may be sufficient to eliminate blood-brain barrier penetration, it may not be fully effective at inhibiting binding to GHSR1a peripherally. Triplet-binding, on the other hand, may be sufficient to eliminate both signaling routes (242). Furthermore, it is possible that ghrelin's effects on energy expenditure and substrate utilization are more sensitive to decreases in GHSR1a-mediated signaling than is food intake (242). If this were the case, a gradual decline in ghrelin levels in response to feeding may first lead to fairly rapid restoration of energy expenditure and carbohydrate utilization, while feeding remains elevated to further top up and maximize energy stores. Given that the animal "knows" that a large food source has become available, the animal can afford to increase energy utilization provided feeding continues. If this were true, then the consequences would be that in humans the ability to quickly and potently reduce acylated ghrelin to appropriate levels to terminate feeding may be an important factor in preventing obesity. Mild reductions in ghrelin levels may return energy utilization back to normal, but may not be accompanied by a reduction in feeding. 
As was the case with the use of multiple monoclonal antibodies, polyclonal antibodies appear more effective than single monoclonal antibodies. Using polyclonal anti-ghrelin antibodies, Bagnasco et al. (243) found that ICV administration in rats reduced cumulative 24-hour feeding and also reduced refeeding after an overnight fast. Direct administration in the arcuate, however, only reduced intake in the early phase of refeeding, and total intake over the full 24 hours of refeeding was not different than control animals (243). Furthermore, intraperitoneal injection mirrored the effects of direct arcuate injection (243). These differences suggest that central ghrelinergic control of feeding is mediated more than just by the arcuate nucleus, and the arcuate's role may primarily involve more immediate, short-term control of energy balance. Indeed, as discussed in Section 1.15, the feeding neurocircuitry of the arcuate appears to be more acutely regulated by metabolic signals than other hypothalamic regions. The results of IP polyclonal antibody treatment matching more closely those of the triplet combination of monoclonal antibodies is expected, as the polyclonal pool has various targets on the ghrelin peptide.

\subsubsection{Hydrolyzing anti-ghrelin antibodies}

Mayorov and colleagues $(240)$ have elaborated on the anti-ghrelin antibody theme by developing a monoclonal antibody, GHR-11E11, capable of increasing the hydrolyzing rate of ghrelin's acyl side-chain ester linkage. This antibody has two methods of action: neutralization of acylated ghrelin through binding, as well as deacylation of active ghrelin to produce desacyl ghrelin. This was accomplished using haptens containing a phosphonate monoester at the serine-3 residue, mimicking the ghrelin transition-state of the hydrolyzing/deacylating reaction. The use of phosphonate monoesters for developing hydrolyzing antibodies is a common technique in hapten design (244). Intravenous administration of GHR-11E11 through the tail vein in mice resulted in a $90 \%$ reduction in acyl ghrelin levels 15 minutes after injection, reducing the acyl:desacyl ghrelin ratio, although with no changes in desacyl ghrelin levels (240). Furthermore, 24 hours post injection, acyl ghrelin levels tended to be lower in treated vs. control animals (240). IV administration also increased energy expenditure during a 24 hour fast, with no changes in substrate utilization indicated by the respiratory exchange ratio (240). Similar to other antibodies as well as to other antagonists of the ghrelin system, 6 hour refeeding was reduced following reintroduction of food. Energy expenditure returned to control levels once refed. While the catalytic efficiency of GHR-11E11 is fairly low, with a second-order rate constant of $18 \mathrm{M}^{-1} \mathrm{~s}^{-1}$, this low efficiency still appears to be sufficient to induce physiological changes (240). 


\subsubsection{Active immunization against acylated ghrelin}

The antibody techniques discussed above are based on a passive method of immunization. Active immunization utilizes the animal's own immune system in order to generate the antibodies, as is usually done with human vaccination. This allows more long-term exposure to the antibody, as the immune system "memorizes" its production after immunization, which can significantly reduce the requirement to maintain treatment over the long-term. Various techniques have been employed to initiate an immune response to ghrelin. There are currently at least three studies examining the use of active vaccination, employing various ghrelin haptens and immunogenic carriers, including keyhole limpet hemocyanin, bovine serum albumin, and NS1 protein tubules from the Bluetongue virus.

Active vaccination has shown very promising results, although data from different species with different vaccination techniques show variability. While results do not always agree, the general pattern is improvements in obesity and/or diabetic conditions. When vaccinated against ghrelin, male and female pigs ate $15 \%$ less food and were $10 \%$ lighter than controls three months after the first inoculation (245). In contrast, while vaccinated rats on standard chow also had reduced weight gain as well as reduced feed efficiency, their food intake was no different from controls (246). Furthermore, although not significant likely due to a small sample size, vaccinated rats display a $40 \%$ reduction in insulin levels, most likely as a result of reduced adiposity and plasma leptin levels (246). Andrade et al. (247) compared active vaccination in regular weight and diet-induced obese mice given ad libitum access to standard chow. In these animals, vaccination led to increased energy expenditure and, in normal weight mice, reductions in food intake (247). These data suggest that active vaccination may provide possible preventative treatment in non-obese individuals identified as being susceptible to obesity, possibly through family history or genetic characterization. Neither group of animals, however, differed from controls in weight gain, and no reductions in food intake were observed in DIO mice. One may suspect that the lack of access to a high-fat diet, variations in the intensity of the immune response, and perhaps the duration of the experiment may be contributors to the absence of these changes. Indeed, Vizcarra et al. (245) and Zorrilla et al. (246) both found a reduction in weight gain in vaccinated pigs and rats, respectively. Zorrilla et al. (246) found that titers were still relatively low, albeit present, up to week eight, and food intake and weight gain were measured up to approximately three months compared to less than two months in Andrade et al's study. Furthermore, Zorrilla et al. (246) found that animals that displayed a stronger immune response to the ghrelin hapten gained less weight and fat mass than those with a weaker immune response. In Andrade and colleagues' (247) study, titers reached a maximum of approximately 1,265, while in Zorrilla and colleagues' (246) study measurements of weight gain, food intake, and feed efficiency 
began once titers reached 10,000 in some rats.

The three methods of active immunization discussed provide promising data for treatment and perhaps prevention of obesity. In each case, increasing titers of antighrelin antibodies were detected, without any evidence of non-specific immune response suggested by a lack of increases in inflammatory signallers, apoptosis, or toxicity. Given that immunization in most cases can last a considerable length of time, including for an entire lifetime, active vaccination against ghrelin must be approached with caution, and considerably more data examining the long-term consequences of such a treatment must be collected. It is furthermore important to note that, given the wide range of functions of ghrelin, complete neutralization is likely undesireable. In translating this to humans, one must be aware of the differences observed in each model, which included rats, mice, and pigs. As with any other treatment, one should expect different results in humans, although in general the results in each model examined are promising, regardless of the differences. In particular, more data must be collected on the effects of vaccination on diabetic parameters. While immunized rats showed a non-significant reduction in insulin, this is likely due to increased leanness. Glucose tolerance tests and insulin sensitivity tests will help elucidate any potential beneficial effects of vaccination for treatment of diabetes. Given that other methods of ghrelin neutralization, GHSR1a antagonization, and GOAT inhibition (as discussed previously) often demonstrate beneficial effects for treatment of diabetes, one would suspect vaccination would also be beneficial.

\subsection{Neutralizing acyl-ghrelin with a spiegelmer}

Spiegelmers are modified aptamer strands designed to bind with high selectivity to desired targets. By incorporating an unnatural form of nucleic acids with L-ribose sugar moieties in place of the natural D-ribose forms found in typical apatamers, resistance to nucleases is increased and hence in vivo stability of these compounds is improved considerably. L-NOX-B11 is one such spiegelmer selected to bind specifically to the acylated form of ghrelin, reducing ghrelin-induced increases in $\mathrm{Ca}^{2+}$ levels in GHSR1aexpressing cells with an $\mathrm{IC}_{50}$ of $5 \mathrm{nM}$ and a $\mathrm{K}_{\mathrm{d}}$ of $35 \mathrm{nM}$ (248). A further modification to L-NOX-B11 is the addition of a polyethylene glycol group attached to the $5^{\prime}$ end to further increase stability. While L-NOX-B11 was raised against the full-length acylated ghrelin peptide, it is capable of binding to the acylated ghrelin(1-5) fragment (248). LNOX-B1 1 also binds to both human and rat ghrelin, which differ only at amino acids 11 and 12 (248). It has also been shown to abolish the in vivo $\mathrm{GH}$ response to $3 \mathrm{nmol}$ IV ghrelin when given at dosages 5 to 10 times ghrelin's molar amount (248). In vivo measurements on ghrelin spiegelmers in mice show a half-life of around 8 hours when administered by IV or IP injection and 12 hours when administered subcutaneously, 
with much of the clearance done renally (249). More recent generations of L-NOXB11 have been generated, including L-NOX-B11-2 and L-NOX-B11-3, the latter of which shows a five-fold higher binding potency compared to L-NOX-B11 (250).

Various studies have examined these spiegelmers' ability to induce beneficial in vivo effects through their neutralization of acylated ghrelin, although compensatory mechanisms appear to be activated relatively quickly. Pretreatment with L-NOX-B11 is capable of inhibiting the short-term (two to four hour) orexigenic effect of peripherally administered ghrelin $(249,251-253)$. This reduced food intake in L-NOX-B11administered animals is attributable to the first half-hour to hour after ghrelin administration, as animals treated with ghrelin plus L-NOX-B11 accelerate feeding relative to controls for the remainder of the four hour time-frame $(249,251)$. With 14 days of subcutaneous treatment to diet-induced obese mice fed a high-fat diet, animals display reduced weight-gain, fat mass, food intake, and feed efficiency (249). Much of this difference appears to occur early on in treatment, as animals initially show a considerable drop in body weight, followed by a gradual recovery to baseline levels by the end of the fourteen day treatment. Importantly, by the end of the fourteen day treatment period, spiegelmer treated animals, while significantly lighter than animals treated with a control spiegelmer, were not significantly lighter than vehicle-treated animals (249). These data suggest that L-NOX-B11 treatment, in addition to possibly other ghrelin neutralizing treatments, may only show short-term beneficial effects, at least in terms of weight gain and food intake, as compensatory mechanisms are quickly activated. In contrast, the active vaccination techniques discussed previously appeared to have a much more prolonged effect. It is possible that part of the compensatory mechanism is to ramp up acylated ghrelin synthesis in response to reduced signaling. In such a case, active vaccination would likely lead to corresponding adjustments in antibody production. Whether a ceiling is eventually reached for antibody production, and how this might affect compensation, is not known. In terms of administration of spiegelmers, increasing the dosage with time would likely not be a desirable approach, as factors such as toxicity and side effects may be amplified.

Data using L-NOX-B11 also demonstrate the importance of ghrelin in survival during periods of low energy availability, as well as recovery from such states. Fasting is associated with a variety of metabolic changes including reductions in glucose, insulin, and lipogenic enzymes. Upon refeeding, restorations in key hepatic lipogenic enzymes FAS, ACC $\alpha$, ATP citrate lyase (ACL), AMPK $\alpha$, and 6-phosphogluconate dehydrogenase occur relative to the fasted state (252). Restoration can be inhibited by administration of L-NOX-B11, and interestingly, L-NOX-B11 can also attenuate in part the decreases induced by fasting. These data suggest that ghrelin plays an important role in both inhibiting lipogenesis during low energetic states as well as restoring lipogenesis during the ensuing recovery. Inhibition of lipogenesis during low energy availability may lead to favoring of fat utilization as an energy source by peripheral 
tissues, leaving glucose utilization for the brain which is highly dependent on glucose as a source of energy. Indeed, GOAT has been shown to promote survival during severe calorie restriction by preventing severe hypoglycemia, an effect likely mediated by acylated ghrelin and growth hormone (234).

Due to a spiegelmer's large size and negative charge, it is unlikely that L-NOX-B11 can cross the blood-brain barrier, and indeed Helmling et al. (248) were not able to detect the spiegelmer in the brain. L-NOX-B11's inability to cross the blood-brain barrier, however, may be beneficial if the ghrelin peptide is present and active in the brain. Previous data obtained from Rimonabant, a CB1 antagonist, has shown serious affective side-effects, which have been attributed to Rimonabant's central actions. Efforts have been made to modify Rimonabant to reduce its ability to cross the bloodbrain barrier in hopes of reducing these effects. Blocking ghrelin's metabolic role in the periphery, including its ability to promote fat storage, while minimizing direct effects in the brain, may in fact be sufficient to allow weight loss or improve glucose control via direct actions on adipocytes and the liver. Central administration of L-NOX-B11 may shed light on any possible consequences of brain-level actions.

While these data do suggest that L-NOX-B11 can potently inhibit acylated ghrelin, it is interesting to note that Becskei et al. (254) have found that it can not inhibit cFos activation in arcuate neurons in fasted mice, as opposed to satiated mice given exogenous ghrelin in combination with L-NOX-B11. There are several differences in these two paradigms that might explain these observations. Firstly, fasting-induced elevations in ghrelin is associated with a myriad array of modifications outside of ghrelin itself, both centrally and peripherally, that are induced by fasting. Becskei et al. (254) suggest that reductions in glucose, insulin, and leptin, as well as increases in orexin signalling from lateral hypothalamic neurons onto arcuate NPY neurons, may contribute to elevations in cFos, as all of these have been found to influence arcuate activity. As such, inhibiting acylated ghrelin will only inactivate one of many sources contributing to arcuate activity. Secondly, given that L-NOX-B11 is administered peripherally and can not cross the blood-brain barrier, the potential for fasting-induced central ghrelin synthesis and activity remains.

\subsection{Inhibiting GOAT and imitating desacyl ghrelin with CF801}

CF801 is a peptide drug developed by our lab, originally designed as a GOAT inhibiting drug but believed to have desacyl ghrelin analog activity. Design of the drug was based on previous published data examining the effect of ghrelin pentapeptides on GOAT activity (159), with important modifications to allow for cell penetration. As discussed previously, Yang and colleagues reported that the pentapeptide GSAFL- 
$\mathrm{NH}_{2}$ had GOAT inhibiting activity in an in vitro assay. Specifically, GSAFL-NH reduced proghrelin acylation by approximately $65 \%$ at $200 \mu \mathrm{M}$ in $50 \mu \mathrm{L}$ reaction mixtures containing $50 \mu \mathrm{g}$ GOAT-containing membrane, $5 \mu \mathrm{g}$ proghrelin His $8,50 \mu \mathrm{M}$ palmitoyl-CoA, and $1 \mu \mathrm{M}\left[{ }^{3} \mathrm{H}\right]$ octanoyl CoA. While effective in this in vitro assay, it is unlikely that GSAFL- $\mathrm{NH}_{2}$ can penetrate the cell membrane to inhibit GOAT in vivo. To address this issue, we attached an amidated trans-activator of transcription (Tat) sequence to the C-terminus of GSAFL- $\mathrm{NH}_{2}$. This is a common technique employed to improve the cell-penetrating abilities of molecules. We furthermore extended the pentapeptide to include the first 10 amino acids of ghrelin, with the serine-3 residue replaced with alanine. While initially this extension was added to increase similarity with ghrelin and to provide an additional spacer between the more important 5-amino acid recognition core at the $\mathrm{N}$-terminus and the Tat peptide, we would subsequently discover that this extension may be crucial for CF801's ability to act as a desacyl ghrelin analog. Indeed, further extending this sequence to include the first 13 amino acids of ghrelin may improve CF801's potency as a desacyl ghrelin analog. This extension would include both the entire sequence of AZP-531 along with the N-terminal GOATinhibiting sequence. Importantly, the replacement of serine-3 with alanine prevents any acylation of CF801 due to the absence of a hydroxyl group. The full sequence of CF801 is GSAFLSPEHQRKKRRQRRR-NH2 . We currently have considerable data, summarized below and presented in detail in the second manuscript reported in this thesis, demonstrating the drug's effects.

After seven days of IP injections of CF801, animals have reduced levels of circulating acylated ghrelin. After only three days of IP injections, mice with ad lib access to both $60 \%$ high fat and regular chow show a reduction in body mass. After two weeks of treatment animals continue to show reduced body mass, attributable to a significant reduction in fat mass with no changes in lean body mass. This reduction in body weight is associated with an increase in regular chow intake and a decrease in high-fat diet intake, and an overall reduction in weight gained per calorie consumed. Additionally, after four days of CF801 treatment, with access to regular chow only, animals fasted for 24 hours show a reduction in total re-feeding within a four-hour period following reintroduction of food.

Although it is believed that acylated ghrelin affects anxiety, after two weeks of treatment with CF801 animals display no differences in an open-field test (255). When exposed to chronic social defeat stress, however, CF801-treated animals consume significantly less high-fat diet, significantly more regular chow, and overall consume significantly less calories compared to vehicle-treated animals (255). Additionally, CF801 treatment in animals exposed to chronic social defeat stress lose more weight compared to vehicle injected animals (255). In both vehicle and non-injected controls, a significant increase in plasma acylated ghrelin occurs when exposed to chronic social defeat stress, whereas in CF801-treated animals no such increase is observed (255). 
While CF801 appears to have GOAT inhibiting activity, our data indicate that its potency is very low, and we do not believe that CF801's potency is high enough to fully explain our weight-loss data in daily-injected mice. Examination of the peptide sequence compared to the desacyl ghrelin analog AZP-531 suggest that CF801 may further act as a desacyl ghrelin analog. In a set of papers published by the same group, Delhanty et al. (162) and Julien et al. (256) have identified amino acids 6 to 13 of ghrelin as an important sequence for desacyl ghrelin activity. CF801 contains six of the eight amino acids of AZP-531, and our preliminary data are in agreement with CF801 acting as a desacyl ghrelin analog. Included in our data are promising results showing CF801's ability to reduce blood glucose levels, an effect consistent with desacyl ghrelin. Overall, data so far suggest that CF801 acts as a GOAT inhibitor, but only at very high concentrations, as well as a desacyl ghrelin analog, although much research remains.

The development of CF801 was preceded by a study, reported here, examining the effects of targeting the ghrelin system through a GOAT inhibitor. Our initial approach involved the use of octanoylated GS(Dap3)FL- $\mathrm{NH}_{2}$, also called octanoylated Dap3ghrelin(1-5), a GOAT inhibitor previously validated in vitro that had not been studied in vivo. The lack of effects observed with this drug led us to GO-CoA-Tat, another GOAT inhibitor. The cost of this drug, however, imposed considerable restraints, leading to the development of our own low-cost drug CF801. As demonstrated by the second manuscript in this thesis, CF801 displayed beneficial effects in treating obesity and points to CF801 as a useful tool in the study of metabolic syndrome and the ghrelin system. Along with these two studies, we additionally examined the effect of knocking-down GOAT specifically in the brain using modified anti-sense strands. 


\section{Chapter 3}

\section{Chronic subcutaneous octanoylated Dap3 ghrelin(1-5) does not alter weight gain, metabolism, or glucose tolerance}

Martin Wellman \& Alfonso Abizaid

\subsection{Introduction}

The peptide hormone ghrelin acts as a key regulator of energy metabolism. Administration of the peptide induces feeding $(17,18,60-66)$ and increases preference for fatty foods $(68,69)$. Ghrelin has also been shown to have a key influence in glucose control. Both ghrelin and its receptor have been found in the pancreatic islets (30, $77,78)$. In terms of diabetes, the effects of acylated ghrelin are negative, contributing to reduced glucose-stimulated insulin release $(33,34)$ and overall increases in plasma glucose levels $(30,79-81)$.

The primary source of circulating ghrelin is the stomach fundus where preproghrelin is translated in X/A-like cells in rats and $\mathrm{P} / \mathrm{D}_{1}$ cells in humans $(10,257)$. Within the endoplasmic reticulum, preproghrelin's signaling peptide is cleaved by signal peptide peptidase to form proghrelin, which undergoes an acylation reaction catalyzed by GOAT, an enzyme found embedded in the ER membrane $(20,128,140)$. Following acylation at the serine-3 residue using dietary MCFAs as a substrate, the C-terminal 
66 amino acids are cleaved to form the mature 28 amino acid acylated ghrelin peptide, which is secreted from the cell into circulation (140-142). The acylation reaction is key to much of ghrelin's activity, including its feeding, adipogenic, and glucose-stimulating effects, as it is a required post-translational modification for ghrelin to bind to its only known receptor, the GHSR1a. Given that the only known target of GOAT is ghrelin, this enzyme has become a key area of interest for inhibiting ghrelin activity in hopes of stemming obesity. Promising results from both GHSR1a and GOAT knockout animals demonstrate some resistance to the obesogenic effect of a high-fat diet (231). In 2008, Yang et al. (159) identified several synthetic ghrelin-based pentapeptides capable of inhibiting GOAT in an in vitro assay in which microsomal fractions provide the source of active GOAT. These crude membrane fractions have to date been the only available source of active GOAT for use in activity assays, as the membrane-bound nature of GOAT makes it difficult to purify the enzyme in its active form.

Yang and colleagues demonstrated that the pentapeptide GSSFL- $\mathrm{NH}_{2}$, consisting of the first five amino acids of ghrelin with an amidated C-terminus, reduced acylation of proghrelin by $50 \%$ at a concentration of $80 \mu \mathrm{M}$ (159). This pentapeptide was itself capable of being acylated by GOAT and would be expected to behave similarly to full-length acylated ghrelin. Substitution of the serine-3 residue with an octanoylated diaminopropionic acid showed only a minimal reduction in inhibitory activity when compared to GSSFL-NH $\mathrm{NH}_{2}$ (159). Coupled with its increased stability and resistance to deacylation due to the amide bond attaching the acyl side chain to the diaminopropionic acid residue (instead of a typical ester linkage found in acylated ghrelin), the diaminopropionic acid-modified octanoylated GS(Dap3)FL- $\mathrm{NH}_{2}$ appears to be a promising candidate for in vivo inhibition of GOAT. With this in mind, we sought out to examine the effectiveness of this peptide in reducing weight gain in mice on either standard chow or a high-fat diet by continuous infusion via subcutaneous osmotic minipumps. We furthermore examined this synthetic peptide's ability to affect energy substrate utilization and glucose tolerance.

\subsection{Methods}

\subsubsection{Animals}

Two cohorts of male CD1 mice ( $\mathrm{n}=40$ per cohort; 25-30 g) were obtained from Charles River (St.-Constant, Quebec). All mice were maintained on a 12/12 light/dark cycle at $21^{\circ} \mathrm{C}$. The first cohort was maintained with ad libitum access to standard chow, while the second cohort was maintained with ad libitum access to a high-fat diet consisting of $60 \% \mathrm{kcal}$ from fat (TD.06414; Harlan Laboratories, Indianapolis, IN). Con-

stant access to water was supplied. Following a 3 -week acclimation period, mice were randomly assigned to sham, vehicle, low dose, and high dose groups ( $\mathrm{n}=10$ per group) 
and surgeries were performed for osmotic minipump implantation, as described below. Body weight was measured daily. Daily food consumption was also measured, but due to problems collecting all food particles spread throughout the cages a proper analysis of consumption was not possible. In order to examine for possible differences due to termination of drug administration, measurements continued beyond the release duration of the minipumps. One animal in the high fat/high dose group was removed due to health issues. All experimental manipulations were approved by the Carleton University Animal Care Committee and adhered to the standards of the Canadian Council for Animal Care.

\subsubsection{Surgery}

Following the three week acclimation period, surgeries were performed for subcutaneous implantation of Alzet osmotic minipumps from DURECT Corporation $(\mathrm{Cu}-$ pertino, CA). Animals were anesthetized with isoflurane supplemented with oxygen and given $1 \mathrm{mg} / \mathrm{kg}$ Metacam prior to surgery. A small incision was made at the upper back to allow insertion of the minipump and incisions closed using nylon sutures. Animals from the first cohort (on standard chow) were implanted with 2-week osmotic minipumps (Model 1002; $0.25 \mu \mathrm{l} / \mathrm{hr}$ ). Due to a lack of effects in the first cohort, animals from the second cohort (on a high fat diet) were implanted with 4-week osmotic minipumps (Model 1004; $0.11 \mu \mathrm{l} / \mathrm{hr}$ ). All drugs were dissolved in a 50\% dimethyl sulfoxide (DMSO)/water solution. Minipumps were filled prior to surgery with vehicle (50\% DMSO in water), low dose octanoylated Dap3 ghrelin (200 $\mu$ M Dap3 ghrelin for the standard chow animals, $400 \mu \mathrm{M}$ Dap3 ghrelin for the high fat diet animals to account for differences in minipump release rates), or high dose octanoylated Dap3 ghrelin (400 $\mu \mathrm{M}$ Dap3 ghrelin for the standard chow animals, $800 \mu \mathrm{M}$ Dap3 ghrelin for the high fat diet animals). Surgeries were performed on sham animals but minipumps were not implanted. Following surgery, weight measurements began after a two-day recovery period.

\subsubsection{Respiratory exchange ratio}

Respiratory exchange ratio (RER) provides an indication of energy substrate utilization, with high levels (at a magnitude of approximately 1 ) indicating primarily carbohydrate utilization and lower levels (at approximately 0.7 ) indicating primarily fat utilization. RER measurements were performed using TSE PhenoMaster/LabMaster metabolic chambers (TSE Systems International Group; Chesterfield, MO). Approximately five days before surgery, animals were allowed to acclimate to the metabolic chambers for 24 hours by placing them individually into the cages. Animals were given ad libitum access to water and to standard chow or high fat diet, according to their respective group. Following acclimation, animals were returned to their home 
cage. Fourteen days following surgery, RER measurements were performed by placing animals individually into the metabolic chambers for a period of 48 hours. Data from the first 24 hours were discarded and analysis was performed on the final 24 hours.

\subsubsection{Glucose tolerance test}

28 days following minipump implantation, glucose tolerance tests were performed on all animals in the high-fat diet cohort. Animals were fasted over-night then given an injection of $20 \%$ glucose in saline at a dosage of $1 \mathrm{~g} / \mathrm{kg}$. A small cut was made at the tip of the tail and blood glucose measured with a blood glucose strip and a Contour glucose meter (Bayer Corporation; Pittsburgh, PA). Measurements were repeated at $15,30,60$, and 120 minutes after the initial measurement.

\subsection{Results}

\subsubsection{Effect on weight gain and RER in standard chow-fed mice}

Repeated measures ANOVA conducted on the standard chow groups indicated that weight gain since day 2 after surgery did not differ between treatment groups throughout the study $\left(\mathrm{F}_{(3,36)}=0.311, \mathrm{p}=0.818\right)$, which continued for 28 days after surgery. On day 14 after surgery, animals were placed in metabolic chambers for 48 hours to measure RER. Measurements were made automatically every 30 minutes. Data from the first 24 hours (the habituation period) were discarded and data from the remaining 24 hours were analyzed. Repeated measures ANOVA indicated that RER did not differ between treatment groups throughout the 24-hour period $\left(\mathrm{F}_{(3,35)}=1.686, \mathrm{p}=0.188\right)$.

\subsubsection{Effect on weight gain, RER, and glucose tolerance in high-fat diet-fed mice}

Similarly to the standard chow cohort, repeated measures ANOVA conducted on the high fat diet groups indicated that weight gain since day 2 after surgery did not differ between treatment groups throughout the study $\left(\mathrm{F}_{(3,35)}=2.217, \mathrm{p}=0.103\right)$, which continued for the full 37-day period after surgery. On day 14 after surgery, animals were placed in metabolic chambers for 48 hours to measure RER. Measurements were made automatically every 30 minutes. Data from the first 24 hours (the habituation period) were discarded and data from the remaining 24 hours were analyzed. Repeated measures ANOVA indicated that RER did not differ between treatment groups throughout the 24-hour period $\left(\mathrm{F}_{(3,35)}=1.261, \mathrm{p}=0.303\right)$.

A repeated measures ANOVA was also conducted on data obtained from the glucose tolerance test. Results indicate that treatment groups did not differ in blood glu- 

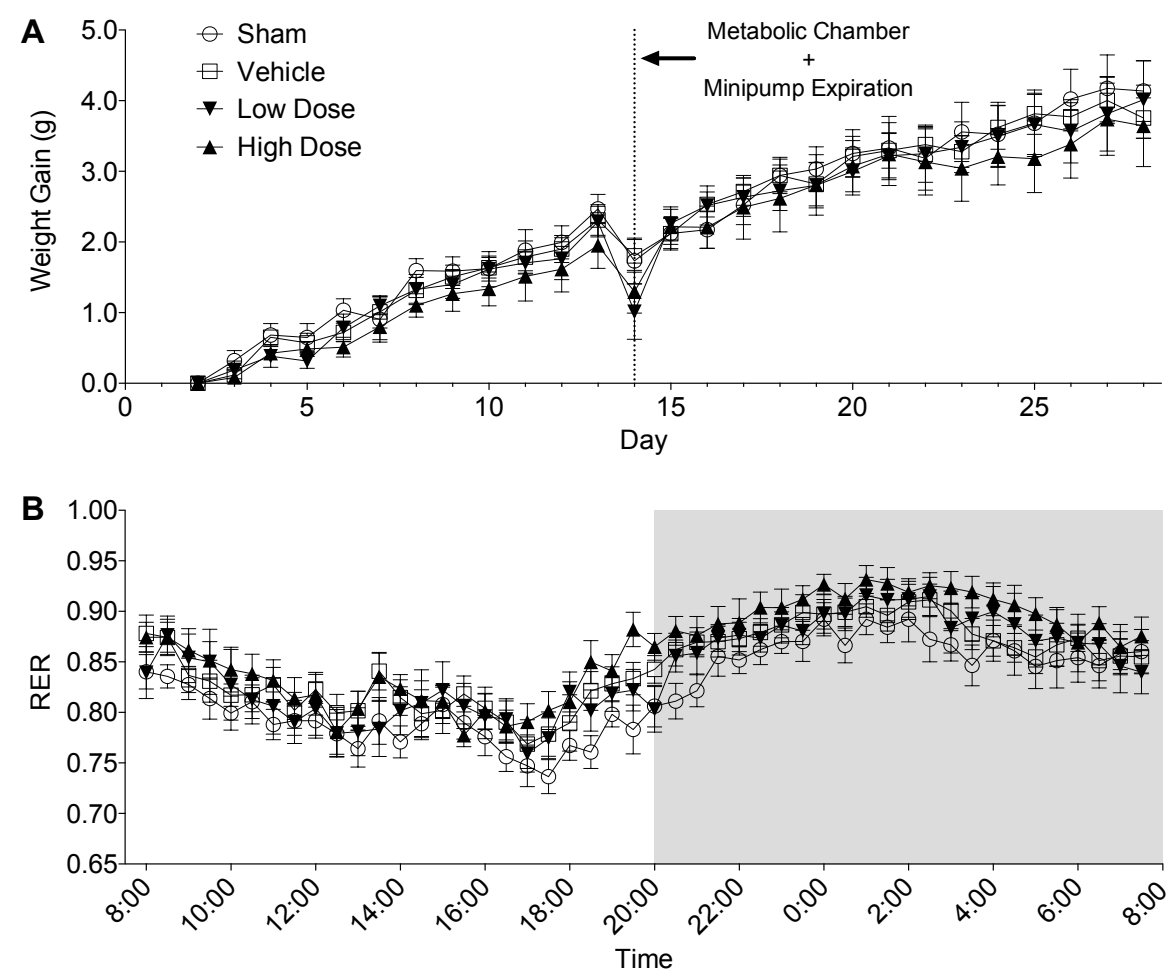

Figure 3.1: Effect of Dap3-ghrelin(1-5) on weight gain and metabolism on standard chow.

Continuous subcutaneous infusion of Dap3-ghrelin(1-5) had no effect on weight gain (A) and 24-hour respiratory exchange ratio (B) on animals with ad libitum access to standard chow. All values are expressed as mean \pm SEM. 

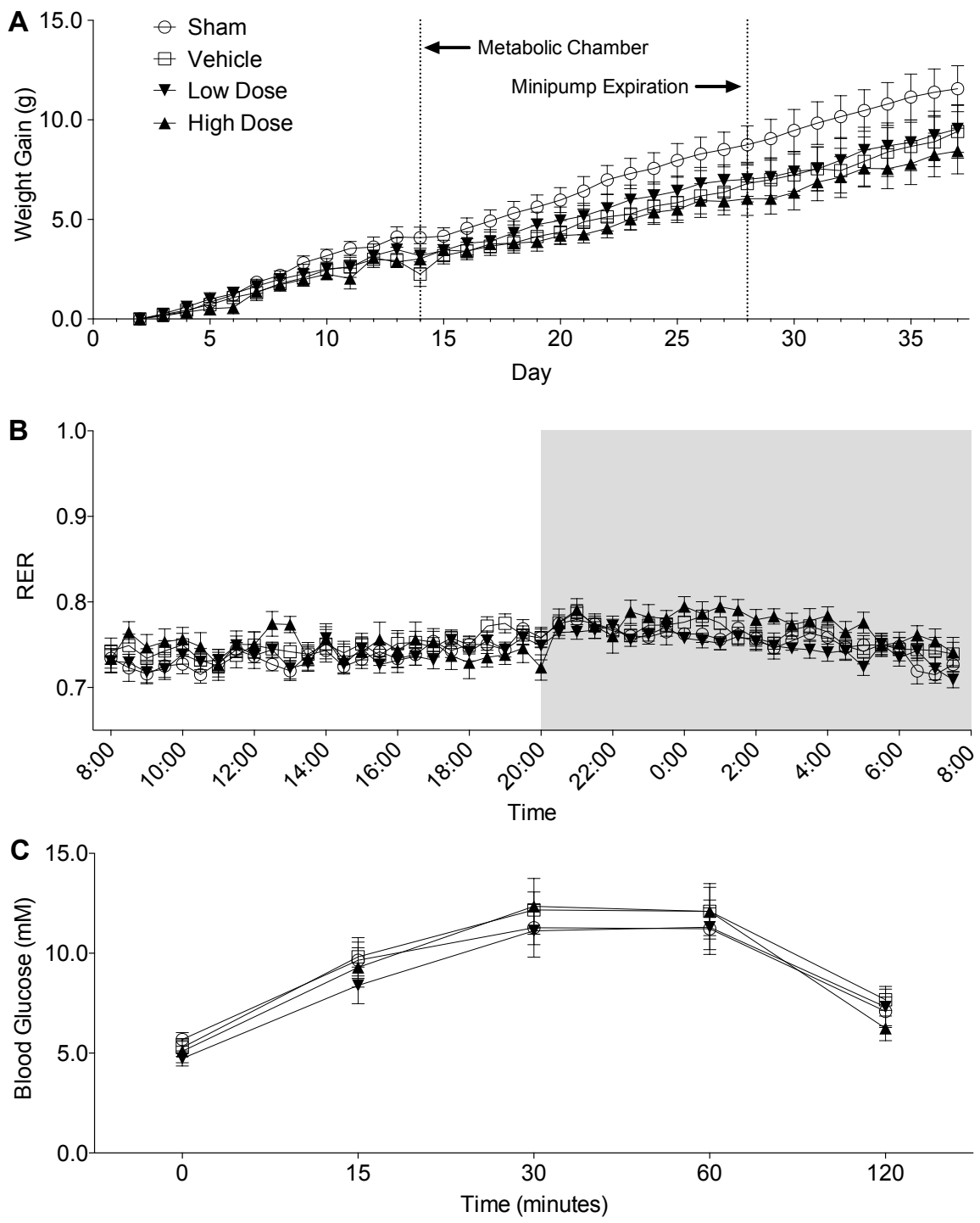

Figure 3.2: Effect of Dap3-ghrelin(1-5) on weight gain, metabolism, and glucose tolerance on a high fat diet.

Continuous subcutaneous infusion of Dap3-ghrelin(1-5) had no effect on weight gain (A), 24-hour respiratory exchange ratio (B), and glucose tolerance (C) on animals with ad libitum access to a high fat diet. All values are expressed as mean \pm SEM. 
cose levels at any of the time points $(0,15,30,60$, and 120 minutes after glucose injection; $\left.\mathrm{F}_{(3,35)}=0.222, \mathrm{p}=0.880\right)$.

\subsection{Discussion}

One of the primary areas of interest surrounding the ghrelin system is its ability to promote weight gain and adiposity. In recent years there have been many attempts at identifying compounds and other treatments that can reduce ghrelinergic activity. These have included small molecule inhibitors, spiegelmers/aptamers, peptidomimetic drugs, and both passive and active immunization. Along with these attempts, various other targets have been examined, such as leptin and the endocannabinoids, with initially promising but ultimately disappointing results. The enzyme GOAT has been a desirable target due to its high selectivity and specificity towards ghrelin, as its only identified role is to acylate the peptide. On the other hand, targeting the ghrelin receptor, which is broadly localized, highly multi-faceted, and appears to have physiological roles even in the absence of ghrelin, seems more prone to side-effects.

Shortly after the identification of GOAT in 2008, Yang et al. (159) developed both a microsomal assay to measure GOAT activity and various pentapeptide GOAT inhibitors that are based on the primary structure of ghrelin. The primary goal of these drugs was to compete for the active site of the enzyme. Among these was octanoylated Dap3 ghrelin(1-5). While octanoylated Dap3 ghrelin(1-5) appeared to work as an effective GOAT inhibitor in Yang and colleagues' (159) assay, its in vivo effectiveness had not been examined. The likelihood that this peptide could cross cell membranes in order to access GOAT enzyme embedded in the ER membrane seems fairly low, and hence its in vivo utility limited. This hypothesis was confirmed in the current study. In Yang et al.'s (159) assay, active GOAT is retained on microsomal membranes. In its natural form, the active site of GOAT is believed to be located within the ER lumen. Due to this topography, it might be necessary for at least some of the microsomes containing membrane-bound GOAT to be in an inside-out orientation, providing access to the GOAT inhibitor. In an intact cellular system, octanoyl-CoA likely makes itself available to GOAT via two possible mechanisms: 1) through simple diffusion through membranes, due to its high hydrophobicity and relatively small size, and/or 2) via an active transport system that may involve the cytosolic Asn-307, which is a highly conserved residue among MBOATs and required for GOAT activity (154).

In the current in vivo study, and in contrast to what would be predicted given data from Yang et al. (159), we did not find any effect of octanoyl Dap3 ghrelin(1-5) on weight gain, substrate utilization, or glucose tolerance in mice maintained on either a high-fat diet or standard chow. This was despite continuous infusion ranging from 14 to 28 days. Although we did not report feeding data due to problems in collecting all 
uneaten food, we nevertheless did analyze the results and found no differences in food intake as well. There are several possible explanations for the lack of an effect. For one, the drug may simply not be an effective GOAT inhibitor. While Yang et al.'s (159) results do suggest that octanoyl Dap3 ghrelin(1-5) does act as a GOAT inhibitor, the conditions of the assay do not exactly match in vivo conditions, and unpublished data using a different HPLC-based assay suggest that octanoyl Dap3 ghrelin(1-5) is at best a weak GOAT inhibitor. Additionally, octanoyl Dap3 ghrelin(1-5) may have difficulty passing through membranes in order to access GOAT. This is quite a significant possibility, and certainly a likely one. The drug GO-CoA-Tat, which is a GOAT inhibitor based on the structure of octanoyl Dap3 ghrelin(1-5), has a crucial modification to the shorter Dap3 pentapeptide in which a Tat sequence is attached to the C-terminus through an aminohexanoic acid linker. Attachment of Tat sequences to peptides is a common technique to increase cell penetrability. Finally, the dosage of the drugs used may be insufficient to result in significant inhibition of GOAT.

\subsubsection{Using a novel approach to reduce GOAT activity}

Our primary suspect to the ineffectiveness of octanoylated Dap3 ghrelin(1-5) in our in vivo experiment was its inability to penetrate cell membranes. While GOAT inhibiting drugs such as GO-CoA-Tat exist, the cost of these drugs for the dosages required for peripheral administration makes these studies infeasible. With this in mind, we developed our own drug named CF801, a low-cost peptide-based drug with modifications to aid in cell penetration. Design of this drug was based on previous data examining various pentapeptide-based GOAT inhibitors, one of which displayed in vitro GOAT-inhibiting activity but with low likelihood of cell permeability. Our addition of an amidated Tat sequence to this peptide was made with the intention to address this problem. Using CF801, which is described in the next chapter, we were able to observe effects not seen in the current experiment. 


\section{Chapter 4}

\section{Novel regulator of acylated ghrelin, CF801, reduces weight gain, rebound feeding after a fast, and adiposity in mice}

Martin K. Wellman, Zachary R. Patterson, Harry MacKay, Joseph E. Darling, Bharath K. Mani, Jeffrey Zigman, James L. Hougland, \& Alfonso Abizaid

Frontiers in Endocrinology 6:144, September 2015

Reprinted with permission from Elsevier Limited

\subsection{Abstract}

Ghrelin is a 28 amino-acid hormonal peptide that is intimately related to the regulation of food intake and body weight. Once secreted, ghrelin binds to the GHSR1a, the only known receptor for ghrelin, and is capable of activating a number of signaling cascades ultimately resulting in an increase in food intake and adiposity. Because ghrelin has been linked to overeating and the development of obesity, a number of pharmacological interventions have been generated in order to interfere with either the activation of ghrelin or interrupting ghrelin signaling as a means to reducing appetite and decrease weight gain. Here we present a novel peptide, CF801, capable of reducing circulating acylated ghrelin levels and subsequent body weight gain and adiposity. To this end, we show that IP administration of CF801 is sufficient to reduce circulating plasma acylated ghrelin levels. Acutely, intraperitoneal injections of CF801 resulted in decreased rebound feeding after an overnight fast. When delivered chronically, CF801 decreased 
weight gain and adiposity without affecting caloric intake. CF801, however, did cause a change in diet preference, decreasing preference for a high fat diet and increasing preference for regular chow diet. Given the complexity of ghrelin receptor function, we propose that CF801 along with other compounds that regulate ghrelin secretion may prove to be a beneficial tool in the study of the ghrelin system, and potential targets for ghrelin based obesity treatments without altering the function of ghrelin receptors.

\subsection{Introduction}

One potential target for the control of body weight and appetite is the gut-brain peptide ghrelin. Ghrelin, a 28 amino acid peptide produced in the X/A-like cells of the gastric oxyntic mucosa lining the stomach, is a key regulator of both short- and long-term energy homeostasis $(9,17,64)$. Circulating ghrelin levels rise in anticipation of a meal and subside once the organism is satiated $(58,258-260)$. Furthermore, ghrelin has been shown to regulate several physiological processes, including glucose metabolism $(80,261-264)$, insulin secretion $(80,265,266)$, gastric emptying (39), cell proliferation $(53,267)$, memory (42), stress $(46,268-270)$, anxiety $(41-43,46,270,271)$ and reward $(69,73,272,273)$. Acutely, ghrelin promotes food intake through interactions with a subset of distinct first order hypothalamic nuclei $(17,61,64,98,274-$ $276)$ and injections of ghrelin result in vigorous feeding bouts (277-279). Ghrelin contributes to long-term energy homeostasis by increasing body weight and adiposity, presumably through a reduction of lipid oxidation $(17,67,269,280)$. Ghrelin increases motivation to obtain food $(69,71,272,273)$ and also increases the preference for highly palatable foods by acting in the VTA $(69,72,272,281)$.

The ability of ghrelin to carry out its physiological processes relies on its affinity for GHSR1a, the only known ghrelin receptor. GHSR1a is widely expressed in both rodents and humans, with highest expression found in the hypothalamus and pituitary gland, both areas heavily invested in energy homeostasis and growth hormone secretion (282). Within the hypothalamus, GHSR1a expression is most concentrated to the arcuate nucleus $(77,282,283)$, a region important in the regulation of food intake, metabolism and energy homeostasis $(17,233,284)$. Interestingly, activation of GHSR1a requires a post-translational modification of the proghrelin peptide (9), a characteristic unique to ghrelin amongst all other metabolically active hormones. Specifically, ghrelin must be acylated on the hydroxyl group of the serine-3 residue with an n-octanoic acid, or other MCFAs containing 6-12 carbons, in order to bind to and activate GHSR1a (9). Modification with an n-octanoyl group induces a conformational flexibility that accommodates the geometric specificity of GHSR1a's binding pocket (158).

The enzyme responsible for the acylation of the mature ghrelin peptide is called 
ghrelin O-acyltransferase (GOAT) $(19,20)$ which belongs to a super-family of enzymes known as the membrane-bound O-acyltransferases (MBOAT) (141). GOAT expression has been demonstrated in many rat, mouse, and human tissues, including the ghrelin producing X/A-like cells of the gastric oxyntic mucosa lining the stomach $(26,138,285)$. To date, GOAT is the only known enzyme capable of acylating, and therefore activating ghrelin for receptor binding, as evidenced by structure-activity analyses of ghrelin mimetic substrates and inhibitors, as well as by studies demonstrating the complete absence of acylated ghrelin in GOAT null mice $(19,161)$. As such, GOAT has become a target for the development of drugs to curb appetite and reduce weight gain via a reduction in acylated ghrelin concentrations $(159,161,235,237$, 286 ). Indeed, a number of compounds that reduce GOAT activity are successful in decreasing weight gain and adiposity. For instance, daily treatment with GO-CoATat, a peptide GOAT inhibitor, was effective in reducing weight gain and adiposity in mice while decreasing acylated ghrelin concentrations (236).

In this paper we present CF801, a synthetic peptide that was thought to be a novel GOAT inhibitor, and one that decreases plasma concentrations of acylated ghrelin in vitro and in vivo. While we demonstrate that CF801 is not an efficient inhibitor of GOAT octanoylation activity, CF801 produced a number of important metabolic effects, including a decrease in weight gain and adiposity associated with changes in appetite and energy expenditure associated with decreased ghrelin concentrations.

\subsection{Methods}

\subsubsection{Design of CF801}

An approach was undertaken to design a novel GOAT inhibitor, resulting in a compound we named CF801. The first five amino acids of CF801 derive from studies carried out in 2008 by Yang et al., who examined the inhibitory effect of certain pentapeptides (e.g. GSAFL- $\mathrm{NH}_{2}$ ) on GOAT using a microsomal assay (159). While these peptides showed inhibitory activity on GOAT, it was unlikely that the peptide could effectively cross membranes to reach the endoplasmic reticulum lumen, where GOAT performs its enzymatic reactions (20). In synthesizing CF801, we extended and modified the original GSAFL- $\mathrm{NH}_{2}$ sequence with the addition of 5 more amino acids that correspond to amino acids 6-10 of the full-length ghrelin peptide. The addition of these amino acids increases the similarity of CF801 to the non acylated ghrelin peptide sequence, as well as provides an appropriate spacer for attachment of additional modifications. The second modification from the GSAFL-NH 2 peptide was the addition of an HIV Tat sequence. CF801 contains the following Tat sequence: RKKRRQRRR, with an amide group retained on the C-terminus.

The full sequence of CF801 is as follows: GSAFLSPEHQRKKRRQRRR-NH ${ }_{2}$, 
where the $\mathrm{N}$-terminus has the standard free amine group $\left(-\mathrm{NH}_{2}\right)$. Variants of this molecule can be synthesized through the inclusion of (or perhaps exclusion of) additional amino acids from the mature ghrelin protein molecule and/or using variants of the Tat sequence or other cell-penetrating peptides. CF801 was custom synthesized by Peptides International (Louisville, KY).

\subsubsection{Cell-based ghrelin secretion assay}

Ghrelin secretion studies were performed in stomach-derived ghrelinoma (SG-1) cells, cultured as described previously $(287,288)$. Cells were plated at a density of $5 \times 10^{4}$ cells $/ \mathrm{mL} /$ well on to 24 -well plates pre-coated with poly-D-lysine (day 0$)$. On day 2, the cells were treated with the indicated concentrations of test compound CF801, 24 hours ahead of the test period and with $50 \mu \mathrm{M}$ sodium octanoate-bovine serum albumin (BSA), 16 hours ahead of the test period. On day 3, the medium was aspirated and the cells were treated with the same concentrations of CF801 in $500 \mu \mathrm{L}$ serum-free DMEM (Life Technologies, Grand Island, NY) supplemented with $5 \mathrm{mM}$ glucose and $50 \mu \mathrm{M}$ sodium octanoate-BSA. After a 6 hour incubation, the medium was collected, placed on ice and immediately centrifuged at $800 \times \mathrm{g}$ for 5 minutes. Hydrochloric acid was added to the supernatant to achieve a final concentration of $0.1 \mathrm{~N}$ (for stabilization of acyl-ghrelin) and stored at $-80^{\circ} \mathrm{C}$ until analysis. Assays for acyl ghrelin and total were performed using commercial ELISA kits (EMD Millipore Corporation, Billerica, MA) according to the manufacturer's instructions and read in a PowerWave XS Microplate spectrophotometer (BioTek Instruments, Inc. Winooski, VT).

\subsubsection{Fluorescence-based GOAT activity assay}

The effects of CF801 on GOAT activity were examined using a previously validated fluorescence based assay, and in comparison with octanoyl-(Dap3)-ghrelin (1-5)- $\mathrm{NH}_{2}$, a well characterized GOAT inhibitor $(159,160)$. In this assay, membrane fractions from Sf9 insect cells were transfected to express either human (hGOAT) or mouse GOAT (mGOAT). Membrane fractions were thawed on ice and passed through an 18 gauge needle ten times enriched using a previously described method (160). Assays were performed with 20-30 $\mu \mathrm{g}$ membrane protein (for hGOAT) and 60-80 $\mu \mathrm{g}$ membrane protein (for mGOAT), $1.5 \mu \mathrm{M}$ GSSFLC $_{\text {AcDan }}$ peptide substrate, $500 \mu \mathrm{M}$ octanoylCoA, 0-10 $\mu \mathrm{M}$ CF801, octanoyl-(Dap3)-ghrelin (1-5)- $\mathrm{NH}_{2}$, or carrier control (50 $\mathrm{mM}$ HEPES $\mathrm{pH}=7.0$ or DMSO) and $50 \mathrm{mM}$ HEPES $\mathrm{pH} 7.0$ in a total volume of $50 \mu \mathrm{L}$. All components with the exception of the acrylodan labeled peptide substrate were incubated in the reaction vessel for 30 minutes prior to assay initiation. Assays were initiated by addition of the acrylodanylated peptide substrate GSSFLC $_{\text {AcDan }}$. Assays were then incubated at room temperature for one hour and stopped by addition 
of $50 \mu \mathrm{L}$ of $20 \%$ acetic acid in isopropanol. Assays were analyzed by reverse phase HPLC with fluorescence detection. Chromatogram analysis and integration of peptide substrate and product peaks were performed using Chemstation for LC (Agilent Technologies). The trials were run in triplicate, with the $\%$ activity calculated as the integrated fluorescence intensity of the octanoylated product in the CF801 or octanoyl(Dap3)-ghrelin (1-5)- $\mathrm{NH}_{2}$ run normalized by the same quantity for the vehicle-only reaction.

\subsubsection{Animals}

In general, male C57BL/6J mice (The Jackson Laboratory, Bar Harbor, Maine) weighing 20-25 g were used as experimental subjects. Throughout the duration of the studies, mice were housed under standard laboratory conditions and received ad libitum access to standard laboratory mouse chow and tap water. Lights in the facility were set to go on and off at 12 hour intervals with the lights going on at 7:00 AM. In some experiments mice also had ad libitum access to a high fat diet containing 60\% caloric content from fat (TD 06414; Harlan Teklad, Indianapolis, Indiana) in addition to regular chow to measure dietary preferences. All procedures were approved by the Carleton University Animal Care Committee and followed the guidelines of the Canadian Council on Animal Care.

\subsubsection{Effects of CF801 on plasma ghrelin concentrations and rebound food intake in fasted mice}

Mice $(\mathrm{n}=16)$ were singly housed and given free access to water and regular chow for a four day baseline period. After this, mice were assigned to one of three groups: vehicle $(\mathrm{n}=5)$, CF801 low dose $\left(\mathrm{n}=5 ; 11 \mu \mathrm{mol} \cdot \mathrm{kg}^{-1}\right.$ - LD) or CF801 high dose $\left(\mathrm{n}=6 ; 22 \mu \mathrm{mol} \cdot \mathrm{kg}^{-1}\right.$ - HD). These mice were injected intraperitoneally once a day for four days starting at 10:00 AM. Following 4 days of treatment, animals were tested for rebound feeding after a fast. To do this, we removed the food from each cage one hour before lights out (6:00 PM), and food was returned to the cage the following day one hour after the last injection. Food intake was then monitored every hour for four hours to determine the effects of CF801 on rebound feeding. Animals were then sacrificed by rapid decapitation to collect trunk blood and other tissues. Two samples were lost during sample collection, so the number of samples used to measure acylated ghrelin was reduced to $\mathrm{n}=14$.

\subsubsection{Tissue and blood analysis}

Following the treatment or recovery periods, mice were killed by rapid decapitation and plasma and tissue samples were collected. To measure glucose levels, glucose strips 
attached to a Contour glucose meter (Bayer Corp., Pittsburgh, Pennsylvania) were dipped in trunk blood collected before being centrifuged. The remaining trunk blood was collected in EDTA-coated tubes placed on ice and centrifuged at $800 \times \mathrm{g}$ for 15 minutes to separate plasma from red blood cells. Blood plasma was aliquoted separately to avoid multiple freeze/thaw cycles and stored at $-80^{\circ} \mathrm{C}$ until processed. To protect the acylated ghrelin molecule, a $50 \mu \mathrm{L}$ aliquot of blood plasma was treated with 2.7 $\mu \mathrm{L}$ of $1.0 \mathrm{~N}$ hydrochloric acid and $10 \mu \mathrm{L}$ of $100 \mathrm{mM}$ 4-(hydroxymercuric)benzoic acid prior to storage. Plasma acylated ghrelin for all animals was measured using an enzyme-linked immunosorbent assay (ELISA) kit (Millipore). All samples analyzed had a coefficient of variation $<10 \%$.

\subsubsection{Effects of CF801 on food intake, weight gain and adiposity}

A second cohort of mice $(\mathrm{n}=30)$ were acclimated to the laboratory conditions for one week prior to onset of the experiment. Mice were housed individually and their body weight and food intake was recorded daily for a baseline period of 10 days. Given that ghrelin augments the intake of preferred diets (272), all mice received ad libitum access to a high fat diet $(60 \%$ of calories coming from fat) in addition to regular chow, to determine if CF801 altered dietary preference. Both chow and high fat diet pellets were provided daily to mice ad libitum in pre-weighed amounts. Mice were then assigned to four different groups: non-injected control (NIC; $\mathrm{n}=7$ ), vehicle ( $0.9 \%$ saline) (VEH; $\mathrm{n}=7)$, low dose CF801 (11 $\left.\mu \mathrm{mol} \cdot \mathrm{kg}^{-1}-\mathrm{LD} ; \mathrm{n}=8\right)$ and high dose CF801 (22 $\left.\mu \mathrm{mol} \cdot \mathrm{kg}^{-1}-\mathrm{HD} ; \mathrm{n}=8\right)$. Each day at 09:00hrs, mice were weighed and their food intake (both chow and high fat diet) was recorded. After this, mice received an intraperitoneal injection of either CF801 or saline (except for the non-injected controls). Following the 13-day treatment period, half the animals in each group were sacrificed while the remaining mice were allowed to recover from treatment. During the 8-day recovery period, mice were granted ad libitum access to standard laboratory chow, high-fat diet and water but did not receive any injections. At this point, the remaining mice were sacrificed. Carcasses from all animals were frozen and stored at $-80^{\circ} \mathrm{C}$ until they were scanned for body composition analyses. Immediately prior to analysis, all carcasses were thawed to room temperature and weighed in order to obtain measures relative to total carcass mass. EchoMRI carcass analyses were performed at Health Canada, Nutrition Research Division facilities using the EchoMRI-1100 (System ID EF-020) scanner. Each carcass was analyzed separately and measurements of total fat mass, total lean mass and total water weight were obtained for each mouse.

\subsubsection{Effects of CF801 on energy expenditure}

Mice ( $\mathrm{n}=10)$ were housed individually and their body weight and food intake was recorded daily for a baseline period of 10 days. As in Experiment 1, all mice had ad 
libitum access to regular lab chow and a high fat diet ( $60 \%$ of calories coming from fat). At the end of the baseline, mice were housed in metabolic chambers (TSE Systems) for 48 hours to examine metabolic rate using indirect calorimetry prior to the onset of the drug treatment. While indirect calorimetry measures were obtained throughout the 48 hour period, we only analyzed measurements in the last 24 hours to allow the animals to adjust to the chambers. Mice were then assigned to one of two groups: vehicle $(0.9 \%$ saline $)(\mathrm{VEH} ; \mathrm{n}=5)$, and CF801 $\left(22 \mu \mathrm{mol} \cdot \mathrm{kg}^{-1} ; \mathrm{n}=5\right)$. Each day at 09:00hrs, mice were weighed and their food intake (both chow and high fat diet) was recorded, and then received an intraperitoneal injection of either CF801 or saline. Drug treatment continued for 16 days. On the 8th day, mice were again placed in the metabolic chambers for 48 hours while still being monitored and injected with their respective treatment to determine differences in metabolism produced by the drug.

\subsubsection{Statistical analyses}

In most experiments, group differences were analyzed using independent samples ttests or one-way ANOVAs followed by Fisher's LSD post hoc tests when significance was reported. Food Intake and weight gain data over time were analyzed using repeated measures ANOVAs followed by post hoc one-way ANOVAs and Fisher's LSD.

\subsection{Results}

\subsubsection{CF801 inhibits acyl ghrelin secretion In Vitro and In Vivo}

SG-1 cells are derived from stomach ghrelinomas induced by expression of the SV40 large T-antigen under the preproghrelin promoter. These cells retain many of the features of ghrelin cells within primary cultures of gastric mucosal cells from mice (288). They also show elevated expression of both preproghrelin and GOAT mRNA, with high levels of acylated ghrelin synthesis and secretion (288). In order to test CF801's ability to reduce acyl ghrelin secretion, we performed an in vitro cell based assay using an SG-1 cell line as previously described $(287,288)$. No significant effects were found after a six hour incubation period with CF801 $(p>0.05)$. However, following a 24hour incubation, CF801 inhibited acyl ghrelin secretion from SG-1 cells with an IC 50 of $347 \pm 40 \mu \mathrm{M}$, based on log-dose vs response non-linear fit. Significant decreases in secretion were found at concentrations of $100 \mu \mathrm{M}(p<0.05)$ and $300 \mu \mathrm{M}(p<0.05)$ CF801 (Figure 4.1).

To determine if CF801 also altered acylated ghrelin concentrations we conducted a study where mice were injected daily with saline or CF801 at one of two doses for four days. No effects of CF801 on food intake or weight gain were observed during the first three days of treatment (data not shown, $p>0.05$ ). Before the last day of treatment, 

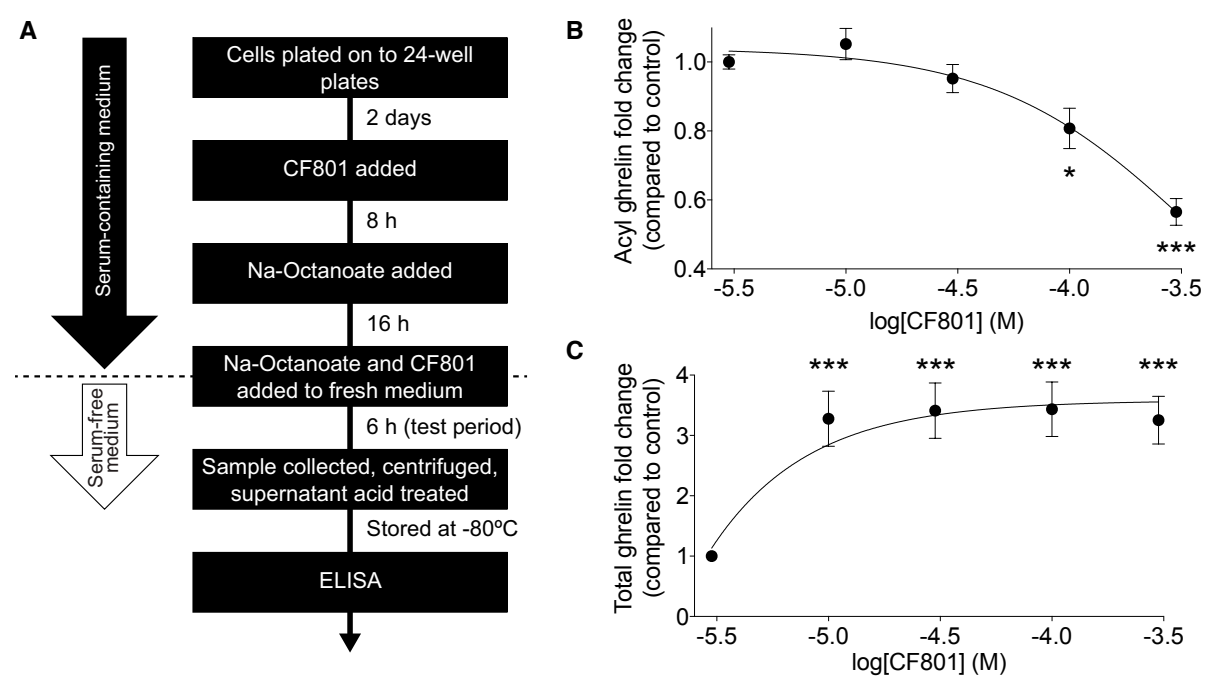

Figure 4.1: CF801 inhibits acyl ghrelin secretion from SG-1 cells following 24 hours of incubation.

Acyl ghrelin concentration in the culture medium collected after $6 \mathrm{~h}$ test period in the presence of the indicated concentrations of CF801. All values are expressed as mean \pm SEM. ${ }^{*} p<0.05,{ }^{* * *} p<0.001$ significant difference in acyl-ghrelin concentrations with CF801 treatment when compared to untreated control, $n=6$ wells. 
A

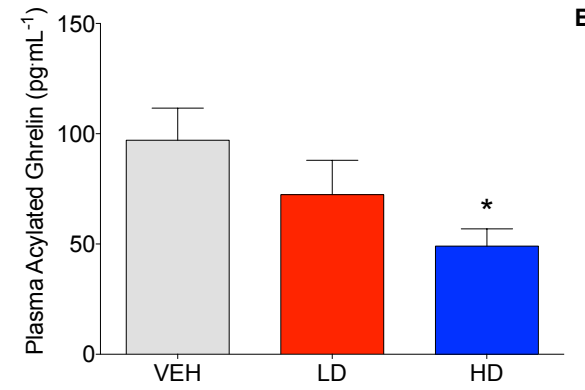

B

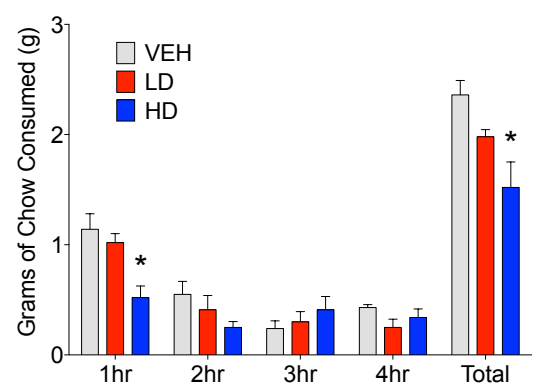

C

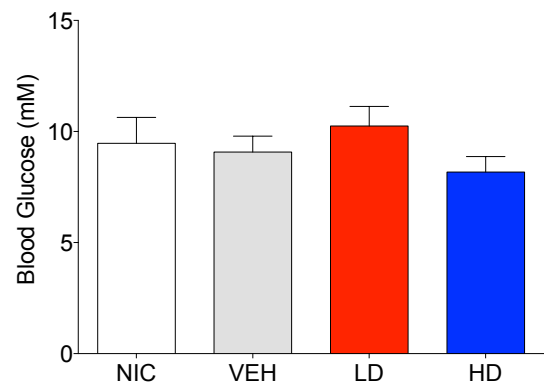

Figure 4.2: Effect of CF801 on plasma acylated ghrelin, chow consumed, and blood glucose.

Animals given HD CF801 displayed reduced levels of plasma acylated ghrelin following a 24 hour fast compared to vehicle-treated animals (A). Average grams of regular laboratory chow consumed following a 24-hour fast upon refeeding (B), and blood glucose measured from trunk blood (C). All values are expressed as mean \pm SEM. ${ }^{*} p<0.05$ relative to VEH injected controls. 

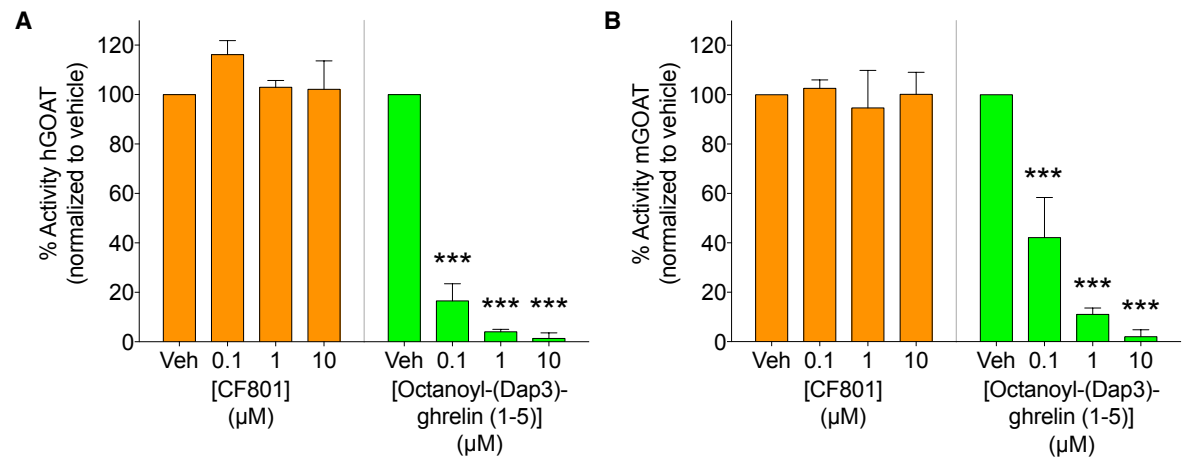

Figure 4.3: Effect of CF801 on hGOAT and mGOAT activity in Sf9 cells.

Percent activity of human GOAT (A) and mouse GOAT (B) (obtained from membrane fractions from Sf9 cells expressing hGOAT and mGOAT, resepectively) when exposed to CF801 and octanoyl-(Dap3)-ghrelin (1-5)- $\mathrm{NH}_{2}$ using a fluorescence-based microsomal assay. GSSFLC $\mathrm{AcDan}_{\mathrm{n}}$ was used as an acylation target with fluorescence measured by reverse phase HPLC with fluorescence detection. CF801 had no effect on either hGOAT or mGOAT acylation activity, while octanoyl-(Dap3)-ghrelin (1-5)- $\mathrm{NH}_{2}$ dose-dependently reduced GOAT activity. All values are expressed as mean \pm SEM. ${ }^{* * *} p<0.001$ compared to vehicle control.
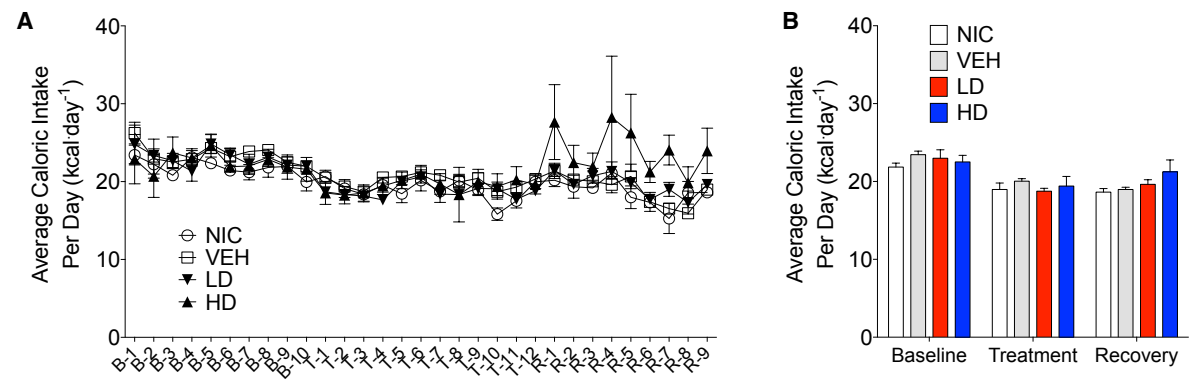

Figure 4.4: Effect of CF801 on caloric intake.

Average daily (A) and overall average (B) caloric intake during the baseline, treatment and recovery period. All values are expressed as the mean \pm SEM. 
A

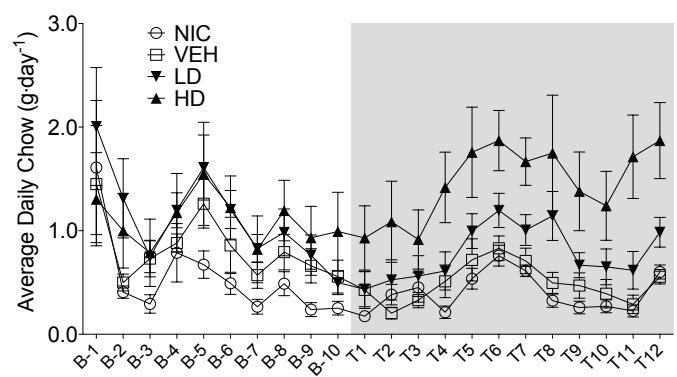

C

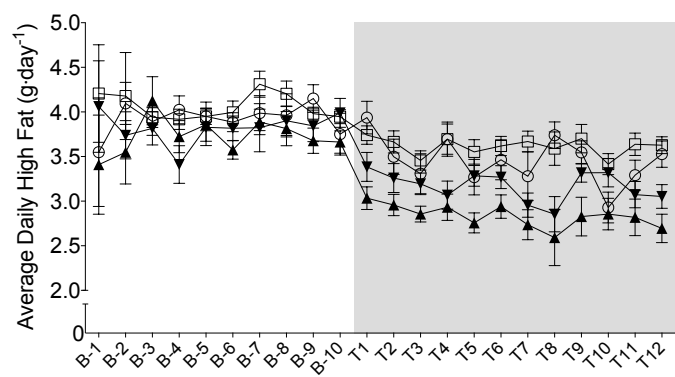

E

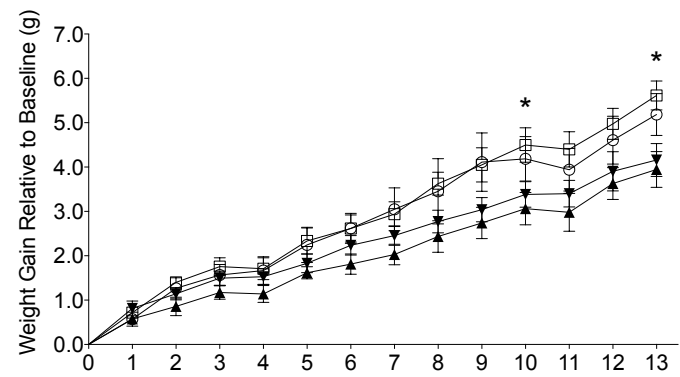

B

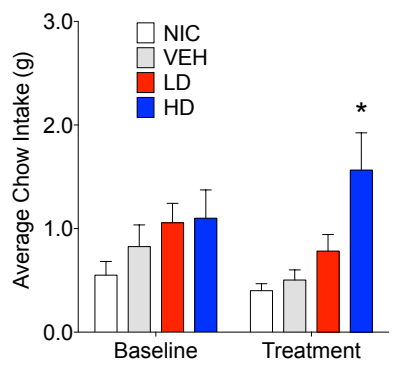

D

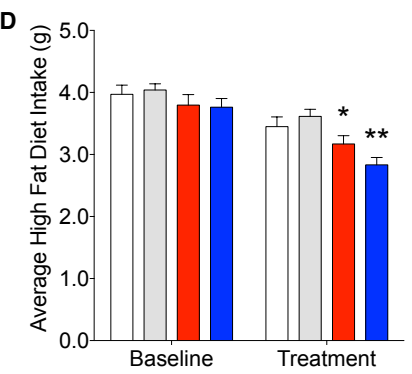

F

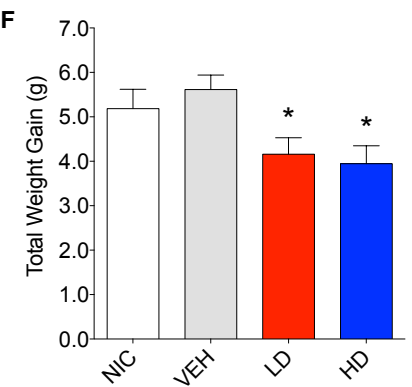

Figure 4.5: Effect of CF801 on food intake and weight gain.

Average daily (A) and overall average (B) chow consumption during baseline and treatment periods. Average daily (C) and overall average (D) high-fat diet consumption during baseline and treatment periods. Weight gain since end of baseline (E) and total weight gain at end of thirteen-day treatment period $(\mathrm{F})$. All values are expressed as means \pm SEM. ${ }^{*} p<0.05,{ }^{* *} p<0.01$ compared to VEH treated controls, Figure E: ${ }^{*} p<0.05$ for HD and LD vs. VEH. 

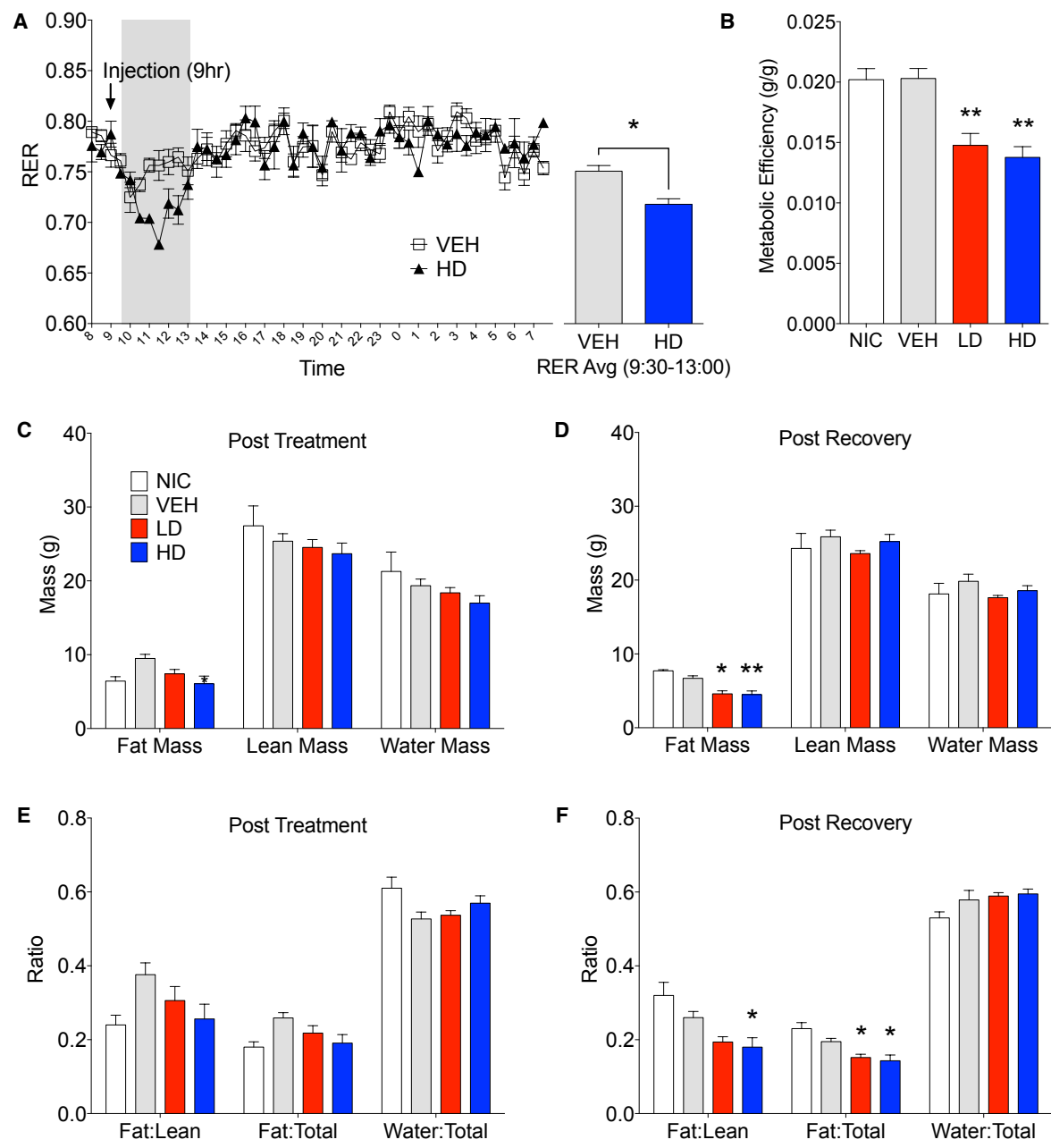

Figure 4.6: Effect of CF801 metabolism and body composition.

Twenty-four hour respiratory exchange ratio following 8 days of vehicle and CF801 (high dose) treatment (A, left) and average RER during the four-hour period following injection at 09:00hrs. (A, right). Metabolic efficiency (B); fat mass, lean mass and water mass following treatment period $(\mathrm{C})$ and recovery period $(\mathrm{D})$; fat mass:lean mass, fat mass:total mass and water mass:total mass following treatment period (E) and recovery period (F). All values are expressed as mean \pm SEM. ${ }^{*} p<0.05,{ }^{* *} p<0.01$ compared to VEH treated animals. 
mice were fasted overnight, treated with their last injection, given access to food for four hours and then sacrificed. Figure 4.2a shows plasma acylated ghrelin concentrations in mice treated with i.p. injections of saline, LD or HD of CF801. As shown in the figure, CF801 caused a dose dependent decrease in plasma ghrelin concentrations that was statistically significant $\left(F_{(2,24)}=2.90 p<0.05, \eta_{p}^{2}=0.22\right)$ in mice treated with the high dose $(p<0.05$ Fisher's LSD). This decrease in acylated ghrelin was also associated with a lower amount of food consumed by mice treated with the high dose of CF801 during the first hour of refeeding (See Figure 4.2b; $F_{(2,24)}=2.394, p<0.05$, $\left.\eta_{p}^{2}=0.22, p<0.05\right)$. This was in correlation with acyl ghrelin concentrations $(\mathrm{r}=0.492$, $p<0.05)$. No significant effects were seen at any other time point examined $(p>0.05)$. Nevertheless, there was a significant overall dose-dependent treatment effect on the total amount of chow consumed during the 4 hour period of food access after the fast $\left(F_{(2,24)}=4.527, \eta_{p}^{2}=0.411 ; p>0.05\right)$. This effect was statistically significant when examining differences in total chow consumed between the HD and saline treated groups ( $p<0.01$, Fisher's LSD). No significant differences were observed between the groups in blood glucose concentrations $\left(\mathrm{F}_{(1,15)}=1.105, p>0.05\right.$; see Figure 4.2c).

\subsubsection{CF801 does not serve as an effective inhibitor of ghrelin octanoylation by GOAT}

To test the potential that CF801's ability to reduce acyl ghrelin secretion in SG-1 cells is due to direct inhibition of GOAT-catalyzed ghrelin octanoylation, we incubated CF801 with both the human and mouse isoforms of the GOAT enzyme under assay conditions previously described (160), with the potent GOAT inhibitor octanoyl(Dap3)-ghrelin (1-5)- $\mathrm{NH}_{2}$ (159) examined in parallel as a positive control for GOAT inhibition. In this assay we tested concentrations comparable with those reported for GO-CoA-Tat, which was reported to have an $\mathrm{IC}_{50}$ for GOAT of less than $1 \mu \mathrm{M}(236)$. At concentrations up to $10 \mu \mathrm{M}$ no inhibition was seen with CF801 incubation (See Figure 4.3; hGOAT: $F_{(3,8)}=1.251, p>0.05$; mGOAT: $\left.F_{(3,8)}=0.139, p>0.05\right)$, while robust inhibition was seen with the control $\left(\mathrm{IC}_{50}\right.$ of $20 \pm 2 \mathrm{nM}$ and $80 \pm 10 \mathrm{nM}$ with hGOAT and mGOAT, respectively; hGOAT: $F_{(3,8)}=485.023, p<0.05$, all doses less than vehicle with $p$ 's $<0.05$ by Fisher's LSD post-hoc; mGOAT: $F_{(3,8)}=51.476$, $p<0.05$, all doses less than vehicle with $p$ 's $<0.05$ by Fisher's LSD post-hoc). This suggests that the observed effects of CF801 in cell- and animal-based studies are not caused by direct inhibition of ghrelin octanoylation by GOAT. 


\subsubsection{CF801 treatment reduces high-fat diet intake and body weight gain without affecting caloric intake}

Given that CF801 decreased ghrelin concentrations and rebound feeding, we decided to examine if CF801 affected weight gain and food intake in mice that had access to a high fat diet in addition to regular chow. As illustrated in Figure 4.4, there were no differences between any groups in the amount of calories consumed during the baseline period $\left(F_{(1,29)}=0.586, p>0.05\right)$, treatment period $\left(F_{(1,29)}=0.783, p>0.05\right)$ and recovery period $\left(F_{(1,29)}=2.093, p>0.05\right)$. It was evident, however, that mice preferred the high fat diet during the baseline period as they consumed a higher proportion of calories from this diet than the calories consumed from the regular chow diet $(p<0.05$; See Figure 4.5). This diet preference changed during the treatment period. In particular, animals receiving high-dose of CF801 consumed significantly more regular chow than animals in every other group as revealed by a one-way ANOVA followed by Fisher's LSD post hoc (see Figure 4.5a and b; $F_{(3,28)}=5.305, p<0.05, \eta_{p}^{2}=0.41$ ). In contrast, CF801 caused an overall significant dose dependent decrease in the intake of the high fat diet (Figure 4.5c and d; $F_{(3,29)}=6.788, p<0.05, \eta_{p}^{2}=0.45$ ). There were no differences in high-fat diet consumption between NIC animals and VEH treated animals. Animals receiving LD CF801 treatment consumed significantly less high-fat diet compared to both NIC and VEH groups $(p$ 's $<0.05)$. Animals receiving HD CF801 consumed significantly less high-fat diet compared to both NIC and VEH groups $(p$ 's $<0.05)$ and tended to consume less than animals receiving LD CF801 $(p=0.073)$. Interestingly, in correlation with decreased intake of the high calorie diet, mice treated with the high dose of CF801 showed a decrease in weight gain compared to mice treated with saline or mice that were not injected at all as determined by a one way ANOVA followed by Fisher's LSD post hoc tests (Figure 4.5e and f; $F_{(3,29)}=3.098$, $\left.p<0.05, \eta_{p}^{2}=0.271\right)$. Mice given the low dose of CF801 tended to have lower weight gain than control NIC $(p=0.092)$ and VEH treated mice $(p=0.065)$, but this difference did not attain statistical significance. Nevertheless, post hoc tests also revealed that there were no differences in weight gain between mice treated with the low dose versus mice treated with the high dose of CF801 $(p>0.05)$.

\subsubsection{CF801 increases the utilization of fat as fuel}

Given that treatment with CF801 decreased weight gain without altering caloric intake, we reasoned that perhaps CF801 was exerting its effects at the metabolic level. To examine this we examined differences in the respiratory exchange ratio (RER) of mice treated with the high dose of CF801 $(n=5)$ with that of saline treated mice $(n=5)$ after a week of daily injections. While both groups of mice showed nearly identical RER during baseline period $(p>0.05)$, mice treated with CF801 displayed a significant decrease in RER during the four hour period that followed the injection of the drug, an 
effect not seen in vehicle treated mice $\left(t_{(5)}=3.457, p<0.05\right)$ (See Figure 4.6a).

\subsubsection{CF801 treatment alters body composition}

Metabolic efficiency, defined as the amount of weight gained per calorie of energy consumed during a given time period, was calculated for each mouse during the baseline and treatment period. There were no differences in metabolic efficiency between any groups during the baseline period (data not shown). As illustrated in Figure 4.6b, there was a significant treatment effect on metabolic efficiency throughout the treatment pe$\operatorname{riod}\left(F_{(1,29)}=9.086, p<0.05, \eta_{p}^{2}=0.522\right)$. Post hoc analyses revealed that animals receiving LD CF801 had significantly lower metabolic efficiencies during the treatment period compared to both NIC $(p<0.05)$ and VEH treated animals $(p<0.05)$. Similarly, animals receiving HD CF801 showed significantly lower metabolic efficiencies during the treatment period compared with both NIC $(p<0.05)$ and VEH treated animals $(p<0.05)$, but were no different than animals receiving LD CF801 $(p>0.05)$.

Given these changes in metabolic efficiency, as well as the decrease in RER observed in the calorimetry experiment, we hypothesized that CF801 treatment would result in less accumulation of fat. To determine if this was the case, we conducted EchoMRI analyses on every animal post-mortem to identify differences in body composition as a function between groups. As shown in Figure 4.6c and d, the amount of total fat mass was significantly lower in mice treated with the high dose of CF801, compared to VEH injected animals right after the treatment period $\left(F_{(1,14)}=3.54, p>0.05 ; \eta_{p}^{2}=0.515\right)$ and this decrease was more evident two weeks after the treatment period was terminated $\left(F_{(1,15)}=15.18, p<0.05 \eta_{p}^{2}=0.805\right)$. Mice receiving LD CF801 treatment and sacrificed at the end of the treatment period had lower fat mass compared to $\mathrm{VEH}$ treated animals but this was not statistically significant $(p>0.05)$, but animals from the same group sacrificed two weeks after the last treatment had lower fat mass than mice in the VEH and NIC groups sacrificed at the same time point $(p<0.05)$. There were no differences between the groups in lean body mass or water mass $(p>0.05)$. Interestingly, there was a significant effect of treatment on fat mass:lean mass ratio following the recovery period (See Figure 4.6e and f; $F_{(1,15)}=7.735, p<0.05 ; \eta_{p}^{2}=0.678$ ). An LSD post hoc analysis revealed that animals receiving LD CF801 treatment tended to show a reduction in fat mass:lean mass ratio following the recovery period compared to VEH treated animals $(p=0.058)$ and had a significantly lower fat mass:lean mass ratio compared to NIC animals $(p<0.05)$. Similarly, animals receiving HD CF801 showed a significantly lower fat mass:lean mass ratio compared to both NIC animals $(p<0.05)$ and VEH treated animals $(p<0.05)$, but were no different than animals treated with LD CF801 $(p>0.05)$. Furthermore, there was a significant treatment effect on the fat mass:total mass ratio following the recovery period $\left(\mathrm{F}_{(1,15)}=9.245\right.$, $\left.p<0.05 ; \eta_{p}^{2}=0.716\right)$. LSD post hoc analysis revealed that both LD and HD CF801 
treated animals had significantly lower fat mass:total mass ratios compared to NIC animals $(p$ 's $<0.05)$ and VEH treated animals $(p$ 's $<0.05)$.

\subsection{Discussion}

Given that ghrelin is one of few peripherally circulating hormones capable of stimulating food intake, ghrelin has become a target for the pharmacological control of appetite and weight gain. Many attempts have been made at interrupting the ghrelin signaling system through the inhibition of ghrelin secretion (289), neutralizing the mature ghrelin protein via vaccination $(246)$ or spiegelmer antagonism $(248,249,251)$ and/or interrupting ghrelin signaling at the level of GHSR1a $(46,69,72,269,290)$. An emerging approach is the development of compounds that target GOAT as a potential therapeutic agent for combating weight gain and adiposity $(159,236,237,286)$. There are several advantages of targeting GOAT for the treatment of obesity such as the selectivity to the targeted acylated ghrelin molecule, the uninterrupted constitutive activity of GHSR1a (presumably necessary for appropriate growth hormone secretion) and the up regulation of unacylated ghrelin (UAG). Finally, it has been suggested that UAG may itself be a viable treatment option through counteracting the activity of acylated ghrelin and improving insulin sensitivity (88).

In this paper we show that CF801, a modified decapeptide similar in structure to the ghrelin peptide, reduces weight gain and adiposity without affecting total caloric intake. This is reminiscent of studies reporting that mice with targeted mutations to the gene encoding the message for the GOAT enzyme do not gain as much weight and store less adipose tissue when fed a high fat diet compared to WT mice (233). Similarly, GHSR-null mice that do not respond to the acylated ghrelin product of GOAT enzymatic activity have reduced respiratory quotient and reduced metabolic efficiency, consume less food, and put on less weight, particularly in the form of adipose tissue, when given a high fat diet (18). These findings are also similar to those from studies where mice were treated with GO-CoA-Tat, a previously characterized inhibitor of GOAT activity (236). Unlike GO-CoA-Tat, the mechanism underlying these effects does not appear to involve direct inhibition of GOAT octanoylation activity although it does involve a reduction in ghrelin secretion. Furthermore, CF801 was able to blunt the usual rebound feeding response to a 24 hour fast, reminiscent to what had been shown previously for a known GHSR antagonist (73).

One way in which CF801 could be decreasing weight gain and adiposity is through a dietary change. Indeed, while CF801 did not alter total caloric intake, it did affect the proportion of calories mice consumed from a high fat diet and those consumed from laboratory chow diet. Looking at the baseline period, one can observe that mice generally preferred to consume the high fat diet, a trend that continued throughout 
the study in control animals. CF801 treated mice, however, reduced their intake of the high fat diet, and increased their intake of chow diet. It is unclear if this change in preference is due to an effect of the drug on preference or palatability, but it is certainly not due to a change in overall appetite as these mice ate enough to maintain their caloric intake throughout the study. It is important to note that at the end of the treatment period, there was a small spike in caloric intake in CF801 treated animals, suggesting that appetite increases once CF801 injections stop. This may indicate that CF801 is associated with enhancing signals that promote satiety, or simply by reducing ghrelin tone, but this needs to be studied further. The latter explanation is supported by data from our first study where we show that mice repeatedly treated with CF801 decrease rebound food intake following an overnight fast in a dose dependent manner and in correlation with acyl-ghrelin concentrations in plasma.

Another way by which CF801 decreases weight gain and adiposity without affecting total caloric intake may be through a change in metabolism. It is well known that ghrelin favors the utilization of carbohydrates as a source of fuels as demonstrated by ghrelin-induced increases in RER in mice receiving daily injections of ghrelin (17). Here we show that repeated injections of the higher dose of CF801 led to acute decreases in RER suggesting that CF801 changes metabolism favoring the utilization of lipids as fuel. This may also explain why CF801 treated mice also showed a lower proportion of body fat with no changes in lean or water mass. This is also similar to the effects of daily injections of GO-CoA-Tat, a treatment that also resulted in a reduction of overall fat mass relative to control vehicle treated mice (236).

As described above, CF801 produces its effects through a reduction in acyl ghrelin concentrations but not through a direct inhibition of GOAT activity. Using an in vitro cell-based assay we were able to detect significant reductions in acyl ghrelin secretion from SG-1 cells following 24 hours of incubation in $100 \mu \mathrm{M}$ and $300 \mu \mathrm{M}$ of CF801. Our preliminary data indicated that at a shorter incubation time of only 6 hours CF801 had no effect on acyl ghrelin secretion (data not shown), even at the highest concentration of $300 \mu \mathrm{M}$. Although Tat-peptide conjugates have been shown to attain maximal penetration within the first 3 hours (291), our specific combination of amino acids has not been directly examined in this regard although time frames considerably above 3 hours seem unlikely. Furthermore, stability of acylated ghrelin within vesicles is not known, but does not preclude the possibility of reserve acylated ghrelin packaged within secretory vesicles. Our data suggest that CF801's effects may not be by direct interaction with the GOAT enzyme. This is also suggested by the lack of potency demonstrated by CF801 in the in vitro microsomal GOAT activity assay that directly assesses GOAT-catalyzed octanoylation of ghrelin mimetic peptide substrates. In contrast to the SG-1 cell-based assay and the effects of CF801 in vivo, CF801 was not able to reduce GOAT activity, while the known GOAT inhibitor octanoyl-(Dap3)-ghrelin (1-5)- $\mathrm{NH}_{2}$ showed a significant reduction. 
Given the high amino-acid similarity between CF801 and desacyl ghrelin, it is possible that CF801 acts like an analog of desacyl ghrelin. Indeed, desacyl ghrelin and the cyclic desacyl ghrelin analog AZP-531 appear to have effects that are similar to those of CF801, including decreases in weight gain, ghrelin secretion, and adipose tissue accumulation $(162,292)$. Alternatively, CF801 may reduce acylated ghrelin simply by reducing GOAT mRNA and expression, without altering GOAT activity. While the mechanisms underlying these effects are not well understood, the idea that CF801 works like desacyl ghrelin or its analogs is an interesting contention as these are currently being proposed for potential clinical treatments to curb obesity. Given that ghrelin may play an important role in attenuating the effects of stress $(46,293,294)$ or those of acute insults (295), more studies are required to determine if reducing acyl ghrelin concentrations with CF801 could have unwarranted side effects. Nevertheless, decreasing acyl ghrelin concentrations peripherally with CF801 or with other bona fide GOAT inhibitors may prove to be a desirable option for the treatment of obesity without affecting the functionality of the ghrelin receptor in the CNS.

\subsubsection{Targeting central GOAT}

In the current study we demonstrated the effectiveness of our own drug at reducing weight gain and altering food intake in mice. These changes were observed with peripheral administration of a drug that was capable of reducing circulating acylated ghrelin levels. Our next goal was to determine if central knock-down of GOAT has effects on metabolism. Previous data, including those obtained from the current study, have not been able to specifically isolate the central role of GOAT, given the peripheral administration of high doses of drugs and the unknown properties of these compounds in terms of blood-brain barrier permeability. Indeed, it is also not known if GOAT is active centrally, or if acylated ghrelin is synthesized in the brain. There is, as will be discussed, evidence that this is true. While CF801 has effects when given peripherally, its mechanism of action is not fully understood and further validation is required. In the next study we therefore took an alternate approach that had not been attempted previously, using vivo-morpholinos directed into the lateral ventricles to centrally knock-down GOAT at the translational level.

\subsection{Acknowledgements}

This project was supported by Funds obtained from the Canadian Institutes for Health Research (CIHR), Natural Sciences and Engineering Research Council (NSERC) and Carleton University (AA), NSERC CGSD Scholarship (ZRP, MKW), Ontario Graduate Scholarship (MKW, HM), the Hilda and Preston Davis Foundation Postdoctoral Fellowship in Eating Disorders Research (BKM), an International Research Alliance 
grant with the Novo Nordisk Foundation Center for Basic Metabolic Research at the University of Copenhagen (JMZ), the Foundation for Prader-Willi Research (JLH), a National Science Foundation Predoctoral Fellowship (DGE-1247399) (JED), and Syracuse University (JED, JLH). We thank Prof. Jef Boeke (NYU Langone Medical Center) for the gift of a plasmid encoding the mouse GOAT isoform. 


\title{
Chapter 5
}

\section{Knockdown of central ghrelin O-acyltransferase by vivo-morpholino reduces body mass of rats fed a high-fat diet}

\author{
Martin Wellman \& Alfonso Abizaid \\ Peptides 70:17-22, August 2015 \\ Reprinted with permission from Elsevier Limited.
}

\subsection{Abstract}

The enzyme GOAT activates the orexigenic peptide ghrelin by transferring an acyl group from fatty acids to the serine- 3 residue of the ghrelin molecule. This allows ghrelin to bind to its only known receptor, the GHSR1a. While studies have examined the hypothalamic transcriptional response of GOAT to metabolic challenge in mice, little has been examined in the rat hypothalamus. Furthermore, it has not been possible to identify the role of central GOAT separate from that of the periphery, since previous studies either knocked out GOAT system-wide or administered a GOAT inhibitor intraperitoneally. To determine if central GOAT expression is modulated by changes in energy state, we subjected rats to either forty-eight hours of food deprivation or three weeks of food restriction and found that GOAT mRNA increases significantly in both the hypothalamus and the stomach fundus in response to both metabolic challenges. We also found increases in hypothalamic ghrelin mRNA and stomach GHSR1a mRNA in response to food deprivation, as well as increases in hypothalamic GHSR1a 
mRNA in response to food restriction. We then conducted a second study where we continuously infused a morpholino antisense oligonucleotide into the lateral ventricles of rats to knock-down GOAT centrally while the animals were exposed to a high fat diet. Our results show that rats receiving the GOAT antisense gained less weight, and decreased their caloric efficiency when eating a high fat diet compared to control animals. These data suggest that central GOAT plays a role in modulating metabolism in rats.

\subsection{Introduction}

Ghrelin is a metabolic hormone produced primarily by the stomach. The metabolic effects of ghrelin include stimulation of growth hormone secretion $(9,61,296)$, increases in food intake and adiposity $(17,61)$, as well as modulation of glucose $(88)$ and lipid metabolism (67). Much of ghrelin's biological activity depends on an esterification reaction in which a fatty acyl side chain is linked to the serine-3 residue, resulting in an acylated ghrelin molecule (9). This side chain, typically but not exclusively involving an 8-carbon octanoyl moiety, is primarily supplied through dietary lipids $(153,233)$. Without this activation, ghrelin cannot bind to its receptor, the GHSR1a $(19,20)$. The esterification reaction is accomplished through the activity of the enzyme GOAT, a member of the group of membrane-bound O-acyltransferases $(19,20)$. Transcripts for GOAT have been found in the stomach $(20,24,26,137$, 138 ) and hypothalamus of both rats and mice with widespread expression in humans (285). Furthermore, ghrelin is the only known hormone requiring octanoylation to be activated (9), making GOAT a desirable target to specifically reduce acylated ghrelin levels and affect such phenomena as food intake and adiposity.

The effects of ghrelin on food intake and adiposity have been attributed to the action of peripheral ghrelin on its receptors in the brain, and particularly in the hypothalamus $(17,60,61)$. There is some debate on the origin of ghrelin binding to hypothalamic and extrahypothalamic target sites, with some authors suggesting that ghrelin is produced within the hypothalamus itself $(9,95,97)$. While the existence of a hypothalamic source of ghrelin has been questioned (297), one would expect that if ghrelin was secreted in the hypothalamus, GOAT would also be synthesized in hypothalamic tissues, and that the expression of this enzyme would be modulated by changes in energy state.

The response of the ghrelin system to short-term food deprivation in mice (in both the stomach and the hypothalamus) as well as to chronic food restriction in rats (in the stomach only) has been reported previously $(24,137,233,298,299)$. Particularly interesting is the fact that GOAT mRNA expression is increased in the hypothalamus of fasted rats (137) although it is not clear if similar increases in the expression of GOAT 
in prolonged energy challenges such as chronic food restriction occur. Nevertheless, the fact that hypothalamic GOAT mRNA expression can be modulated by fasting suggests that this enzyme is important for the regulation of energy balance. Indeed, the expression of GOAT has been linked to changes in energy state. For instance, studies using mice with targeted deletion of the GOAT gene have shown that these mice, like GHSR and ghrelin KO mice, show mild alterations in body weight and food intake (234). These differences however, are more evident when GOAT KO mice are exposed to high fat diets or when exposed to fasting conditions $(233,234)$. Interestingly, a small-molecule GOAT-inhibiting drug named GO-CoA-Tat reduced the excessive weight gain observed in wild-type C57BL6 mice on a diet high in mediumchain triglycerides (236). This reduction in weight occurred quite rapidly, taking only one week for significant differences to occur, and divergence appearing to begin only after about four days on the diet. Nevertheless, while GO-CoA-Tat treated mice are leaner than vehicle treated animals, it is not possible to determine the relative contribution of GOAT produced in the hypothalamus versus that of GOAT synthesized in the stomach in these processes.

Given that the effects of total genetic deletion of GOAT, and those of GO-CoA-Tat are observable in animals exposed to a diet high in triglycerides, here we attempted to further characterize the role of central GOAT on body weight gain and caloric intake in rats exposed to a high fat diet. In the current study, we sought out to first confirm regulation of ghrelin related genes by measurements of ghrelin, GHSR1a and GOAT mRNA in the rat fundus and hypothalamus in response to forty-eight hours of food deprivation and three weeks of food restriction. After verifying that GOAT mRNA is present and can be regulated in the hypothalamus, we then sought out to determine the effects of knocking down GOAT mRNA expression in the brain on measures of food intake and body weight in rats. We accomplished this by providing continuous infusion of vivo-morpholino antisense oligonucleotide (VMO) targeting the rat GOAT sequence for a four-week period into the left lateral ventricle of rats.

\subsection{Methods}

\subsubsection{Experiment 1: Effect of food deprivation and food restriction on GOAT, ghrelin, and GHSR1a mRNA in the fundus and the hypothalamus}

\section{Animals}

Male Long Evans rats $(\mathrm{n}=50)(276-300 \mathrm{~g})$ were obtained from Charles River (St.Constant, Quebec), and kept individually in a $12 / 12$ light/dark cycle at $21^{\circ} \mathrm{C}$, with constant access to standard chow and water before experimental manipulations. After 
a one-week baseline where food and body weight were recorded, rats were randomly assigned to one of three groups: 1) Control ad lib fed rats $(\mathrm{n}=17)$; 2) Rats food deprived for 48 hours ( $n=16)$; and 3) Rats food restricted for three weeks ( $n=16)$. Food restricted animals were kept on a diet consisting of $50 \%$ of the average daily food consumed during baseline. All experimental manipulations were approved by the Carleton University Animal Care Committee and adhered to the standards of the Canadian Council for Animal Care.

\section{Tissue Collection}

Animals in all groups were rapidly decapitated and hypothalamic punches (relative to bregma: AP $-2.3 \mathrm{~mm}$ to $-3.8 \mathrm{~mm}, \mathrm{~L}-1.0 \mathrm{~mm}$ to $+1.0 \mathrm{~mm}$, DV $+7.5 \mathrm{~mm}$ to base) were extracted from the brain and placed in TRIzol (Invitrogen). Stomach fundus samples were also obtained. Brain samples were stored in TRIzol at $-20^{\circ} \mathrm{C}$ and fundus samples at $-80^{\circ} \mathrm{C}$ (not in TRIzol) until processed. Trunk blood was collected in BD Vacutainer $\mathrm{K}_{2}$ EDTA tubes (Franklin Lakes, New Jersey). Blood samples were briefly vortexed, centrifuged at 3000 RPM for 15 minutes in an IEC Centra GP8R centrifuge. Blood plasma was separated and treated with $5 \mu \mathrm{l} 1 \mathrm{~N} \mathrm{HCl}$ and $5 \mu \mathrm{l}$-hydroxymercuribenzoic acid per $0.5 \mathrm{ml}$ of plasma to protect against degradation of acylated ghrelin.

\section{Enzyme-Linked Immunosorbent Assay}

Enzyme-Linked Immunosorbent Assay was conducted on all plasma samples using a Millipore (Billerica, MA) Rat/Mouse Ghrelin (Active) ELISA kit according to the manufacturer's instructions. All samples were run in duplicate in a single assay. Intra assay variability was less than $10 \%$.

\section{RNA Extraction and cDNA Synthesis}

RNA extraction was performed on all brain and stomach samples obtained in both experiments using the TRIzol protocol provided by Invitrogen, using $13 \mu \mathrm{l}$ of linear acrylamide as a co-precipitate with isopropyl alcohol during the RNA precipitation stage. cDNA synthesis was performed on all mRNA samples. Briefly, $1 \mu$ l oligo(dT) primer (Invitrogen) was added to $9 \mu \mathrm{l}$ mRNA and heated at $70^{\circ} \mathrm{C}$ for 5 minutes. To each sample, a master mix of $4 \mu \mathrm{l}$ 5X First-Strand Buffer (Invitrogen), $2 \mu \mathrm{l} 0.1 \mathrm{M}$ DTT (Invitrogen), $1 \mu \mathrm{l} 10 \mathrm{mM}$ dNTP (Invitrogen), $1 \mu \mathrm{l}$ RNase inhibitor (Promega), $1 \mu \mathrm{l} \mathrm{SS} 2 \mathrm{RT}$ (Invitrogen), and $1 \mu \mathrm{l}$ DEPC-treated water were added. Samples were then run on a PTC-200 Thermal Cycler (MJ Research) at $42^{\circ} \mathrm{C}$ for 1.5 hours followed by $90^{\circ} \mathrm{C}$ for 10 minutes. Samples were maintained in the cycler at $4^{\circ} \mathrm{C}$ until stored at $-20^{\circ} \mathrm{C}$. 


\begin{tabular}{|c|c|c|}
\hline Gene & & Primer Sequence $\left(5^{\prime}-3^{\prime}\right)$ \\
\hline \multirow[t]{2}{*}{ Ghrelin } & Forward & CCAGAGGACAGAGGACAAGC \\
\hline & Reverse & AGTTGCAGAGGAGGCAGAAGCT \\
\hline \multirow[t]{2}{*}{ GOAT } & Forward & TGCCACCTGGGTCTTCACTAC \\
\hline & Reverse & ACGCTGCCTCCACCTTCC \\
\hline \multirow[t]{2}{*}{ GHSR1a } & Forward & CCAGAACCACAAGCAGACAGTG \\
\hline & Reverse & GAAGAGGACAAAGGACACCAGG \\
\hline \multirow[t]{2}{*}{ NPY } & Forward & TGGACTGACCCTCGCTCTAT \\
\hline & Reverse & GTGTCTCAGGGCTGGATCTC \\
\hline \multirow[t]{2}{*}{$\beta$-actin } & Forward & TCATGAAGTGTGACGTTGACATCCG \\
\hline & Reverse & CCTAGAAGCATTTGCGGTGCACGATG \\
\hline \multirow[t]{2}{*}{ GAPDH } & Forward & AAGATGGTGAAGGTCGGTGT \\
\hline & Reverse & CTTGCCGTGGTAGAGTCAT \\
\hline
\end{tabular}

Table 5.1: Primer sequences for qRT-PCR

\section{Quantitative RT-PCR}

Quantitative RT-PCR was conducted on all cDNA samples in duplicate to determine fold changes in GOAT, GHSR1a, and ghrelin mRNA levels, using the $2^{-\Delta \Delta \mathrm{C}_{\mathrm{t}}}$ method with the ad libitum group as a baseline control group. Briefly, $5 \mu \mathrm{l}$ of each cDNA sample was added to separate wells in a PCR plate. $2 \mu \mathrm{l}$ of $2 \mu \mathrm{M}$ primer, $3 \mu \mathrm{l}$ of milli-Q water, and $10 \mu \mathrm{l}$ iQ SYBR Green Super Mix with fluorescein (Bio-Rad) were added to each well. The plate was run on a MyiQ Single Color Real-Time PCR Detection System (Bio-Rad) for 30 seconds at $95^{\circ} \mathrm{C}$, followed by 45 cycles of the following settings: 10 seconds at $95^{\circ} \mathrm{C}$ for denaturing, 30 seconds at $55^{\circ} \mathrm{C}$ for annealing, and 20 seconds at $72^{\circ} \mathrm{C}$ for extension. The plate was then run for $1 \mathrm{~min}$ at $95^{\circ} \mathrm{C}$ and $1 \mathrm{~min}$ at $55^{\circ} \mathrm{C}$. A melt curve was constructed by running the plate for 30 seconds at $55^{\circ} \mathrm{C}$ for 41 repeats, increasing the temperature by $1^{\circ} \mathrm{C}$ for each cycle. $\beta$-actin and GAPDH were used as housekeeping genes. Primers used were as shown in Table 5.1.

\subsubsection{Experiment 2: Effect of central GOAT knock-down on body weight and food intake}

\section{Animals}

Male Long Evans rats $(\mathrm{n}=32)(276-300 \mathrm{~g})$ were obtained from Charles River (St.Constant, Quebec), and kept in a $12 / 12$ light/dark cycle at $21^{\circ} \mathrm{C}$. All rats were housed 
individually and given ad lib access to a high fat diet consisting of $60 \% \mathrm{kcal}$ from fat (TD.06414; Harlan Laboratories, Indianapolis, IN) with constant access to water throughout the experiment. Following a one-week baseline, animals were weightmatched and assigned to one of four groups: 1) vehicle control $(n=7), 2)$ reverse control $(n=6), 3)$ negative control $(n=7)$, and 4) GOAT anti-sense treatment $(n=6)$. Six animals were removed from the experiment due to experimental attrition (four due to surgical complications and two due to misplaced cannulae). All experimental manipulations were approved by the Carleton University Animal Care Committee and adhered to the standards of the Canadian Council for Animal Care.

\section{Vivo-Morpholinos}

Vivo-morpholinos were designed by and obtained from Gene Tools LLC (Philomath, OR). Treatment vivo-morpholinos were designed based on accession number NM_001107317.2 (Rattus norvegicus membrane bound Oacyltransferase domain containing 4 (Mboat4), mRNA), with the sequence 5'-GAAAGAAGAACTGGAGCCAATCCAT-3'. This VMO was designed to target the $5^{\prime}$ end, including the start codon, of the GOAT transcript. The reverse of this sequence was used as a reverse control. In addition, a negative control targeting a human beta-globin intron mutation with the sequence 5'-CCTCTTACCTCAGTTACAATTTATA-3' was used. Vehicle animals were given sterile $\mathrm{PBS}$ at a $\mathrm{pH}$ of 7.35. All vivo-morpholinos were diluted to a concentration of $35.90 \mu \mathrm{M}$ in sterile PBS and stored at room temperature until used. A pilot study determined that this VMO was effective in reducing hypothalamic GOAT mRNA expression to an average of $40.69 \% \pm 14.6 \%$, and NPY mRNA expression to an average of $63.42 \% \pm 8.86 \%$ of vehicle infused animals. Similarly, the VMO reduced GOAT and NPY expression to $45.15 \% \pm 16.20$ and $28.48 \% \pm 10.22$ of the reverse sequence morpholino (negative control). There were no differences in hypothalamic expression of GOAT or NPY mRNA between rats treated with vehicle and reverse sequence morpholino negative controls.

\section{Surgery}

Following the one-week baseline, surgery was performed for continuous infusion of vehicle or the corresponding vivo-morpholino, at $0.25 \mu \mathrm{l} /$ hour, by way of subcutaneous 4-week Alzet osmotic mini-pumps obtained from DURECT Corporation (Model 2004; Cupertino, CA). Animals were anesthetized with isoflurane supplemented with oxygen and given $0.1 \mathrm{ml}$ subcutaneous Metacam prior to surgery. Minipumps were attached via tubing to a cannula targeting the left lateral ventricle (relative to bregma: AP $-0.08 \mathrm{~mm}, \mathrm{~L}+0.16 \mathrm{~mm}, \mathrm{DV}-0.36 \mathrm{~mm}$ ). Animals were given a two-

day recovery period before being handled again. Placement into the ventricles was 
verified by injection of a blue dye and confirmed by the presence of the dye in the third ventricle.

\section{Mass and Food Intake Measurements}

Day of surgery was identified as day 0 . Food intake and rat mass were measured daily at noon from day 0 up to day 17 . Total weight gain and total cumulative food intake were calculated relative to the end of the two-day surgical recovery period (day 2). Repeated measures two-way ANOVAs followed by one-way between groups ANOVAs and Fisher's LSD post-hoc tests were conducted separately for each day to compare, between groups, total percentage weight gain as well as total food intake relative to rat mass at day 2, as well as total weight gain over total food intake.

\subsection{Results}

\subsubsection{Experiment 1: Effect of food deprivation and food restriction on GOAT, ghrelin, and GHSR1a mRNA in the fundus and the hypothalamus} Effect of food restriction and deprivation on hypothalamic GOAT, ghrelin, and
GHSR1a mRNA

One-way ANOVAs indicated that differences occurred in the hypothalamus for GOAT $\operatorname{mRNA}\left(F_{(2,45)}=5.466, p<0.05\right)$, ghrelin mRNA $\left(F_{(2,46)}=4.573, p<0.05\right)$, and GHSR1a mRNA $\left(F_{(2,38)}=13.648, p<0.05\right)$ between the ad lib, 48 hour food deprived, and three week food restricted groups (Figures 5.1a, b, and c). Fisher's post-hoc tests revealed that in the hypothalamus, GOAT mRNA was significantly elevated after 48 hours of food deprivation and after 3 weeks of food restriction $(p<0.05)$ compared to $a d$ lib animals. While hypothalamic ghrelin mRNA levels were significantly elevated after 48 hours of food deprivation $(p<0.05)$, hypothalamic ghrelin mRNA expression was not elevated after 3 weeks of food restriction $(p>0.05)$ in relation to control ad lib fed rats. GHSR1a mRNA levels in the hypothalamus did not change in response to 48 hours of food deprivation but increased in response to three weeks of food restriction $(p<0.05)$.

Effect of food restriction and deprivation on stomach fundus GOAT, ghrelin, and GHSR1a mRNA

One-way ANOVAs indicated that differences occurred in the stomach fundus for GOAT mRNA $\left(F_{(2,38)}=4.949, p<0.05\right)$ and for GHSR1a mRNA $\left(F_{(2,36)}=7.350\right.$, $p<0.05)$, but not for ghrelin mRNA between the ad lib, 48 hour food deprived, and three week food restricted groups (Figures 5.1d, e, and f). 

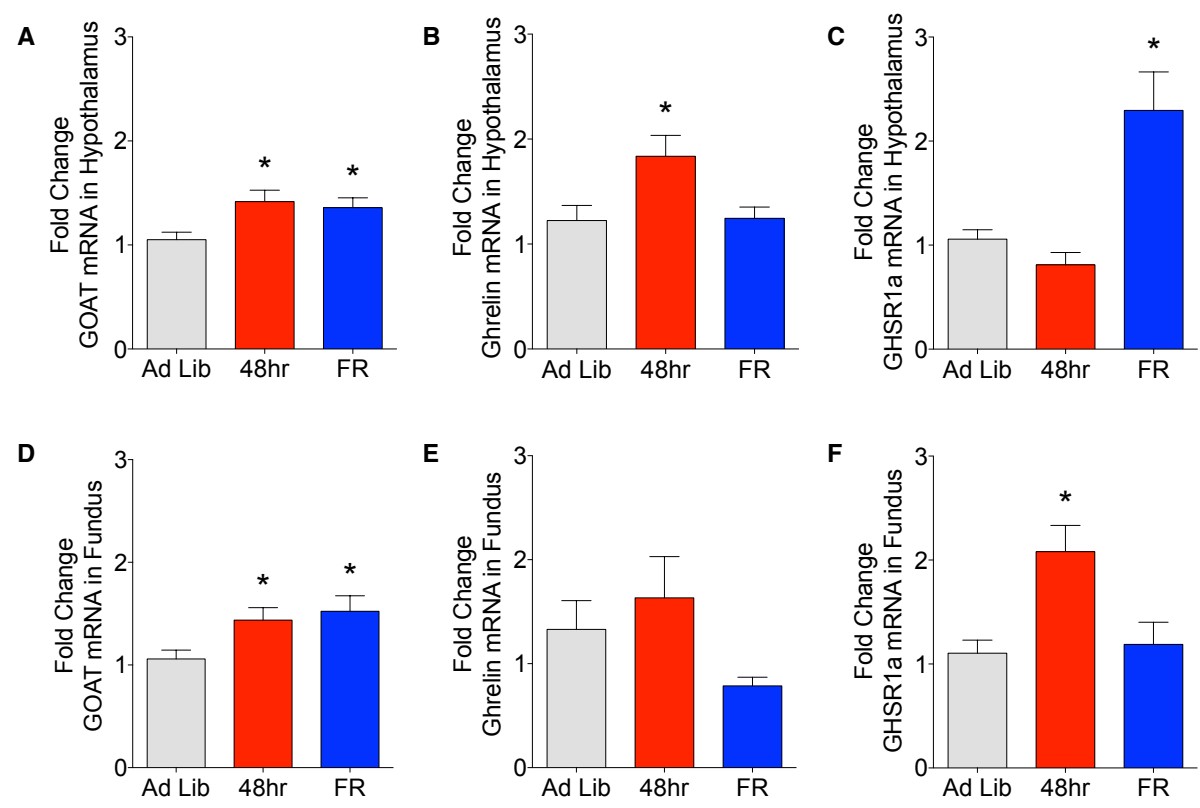

Figure 5.1: qRT-PCR fold changes in the hypothalamus and fundus in response to food deprivation and food restriction.

In the hypothalamus: fold changes in (A) GOAT, (B) ghrelin, and (C) GHSR1a mRNA. In the fundus: fold changes in (D) GOAT, (E) ghrelin, and (F) GHSR1a mRNA. Changes are in response to 48 hours of food deprivation ( $48 \mathrm{hr}$ ) or three weeks of food restriction (FR), relative to $a d$ lib animals (Ad Lib). All data are mean \pm SEM. ${ }^{*} p<0.05$ when compared to ad lib group; by one-way ANOVA with Fisher's LSD post hoc tests. 


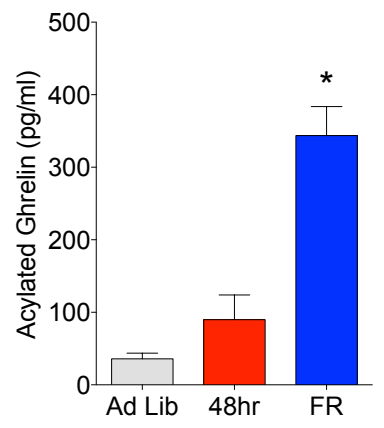

Figure 5.2: Circulating acylated ghrelin levels in response to food deprivation and food restriction.

Plasma levels of active ghrelin obtained from an active ghrelin enzyme-linked immunosorbent assay (ELISA) in ad libitum (Ad Lib), 48-hour food deprived (48hr), and three-week food restricted (FR) animals. All data are mean \pm SEM. ${ }^{*} p<0.05$ when compared to both ad lib and $48 \mathrm{hr}$ groups; by one-way ANOVA with Fisher's LSD post hoc tests.

In the stomach fundus, GOAT mRNA levels followed a similar pattern as in the hypothalamus. Fisher's LSD post-hoc tests revealed that GOAT mRNA significantly increased after 48 hours of food deprivation $(p<0.05)$ as well as after 3 weeks of food restriction $(p<0.05)$. GHSR1a mRNA levels in the fundus increased relative to $a d$ lib animals in response to 48 hours of food deprivation $(p<0.05)$ but no change was found relative to $a d$ lib animals in the three week food deprived animals.

\section{Effect of food restriction and deprivation on circulating acylated ghrelin levels}

A one-way ANOVA $\left(F_{(2,38)}=15.413, p<0.001\right)$ followed by Fisher's LSD post-hocs revealed that plasma levels of active ghrelin were increased in 3-week food restricted animals 9.6 fold relative to ad lib animals (5.2). No significant difference was found between 48-hour food-deprived animals and ad lib animals.

\subsubsection{Experiment 2: Effect of central GOAT knock-down on body weight and food intake}

\section{Effect of Drug Treatment on Body Weight}

Repeated measures two-way ANOVA using the Greenhouse-Geisser correction indicated a significant interaction between day and treatment group on body weight $\left(F_{(9.059,66.435)}=5.217, p<0.05\right)$, indicating that differences in weight gain occurred between treatment groups on different days. One-way ANOVAs followed by Fisher's 

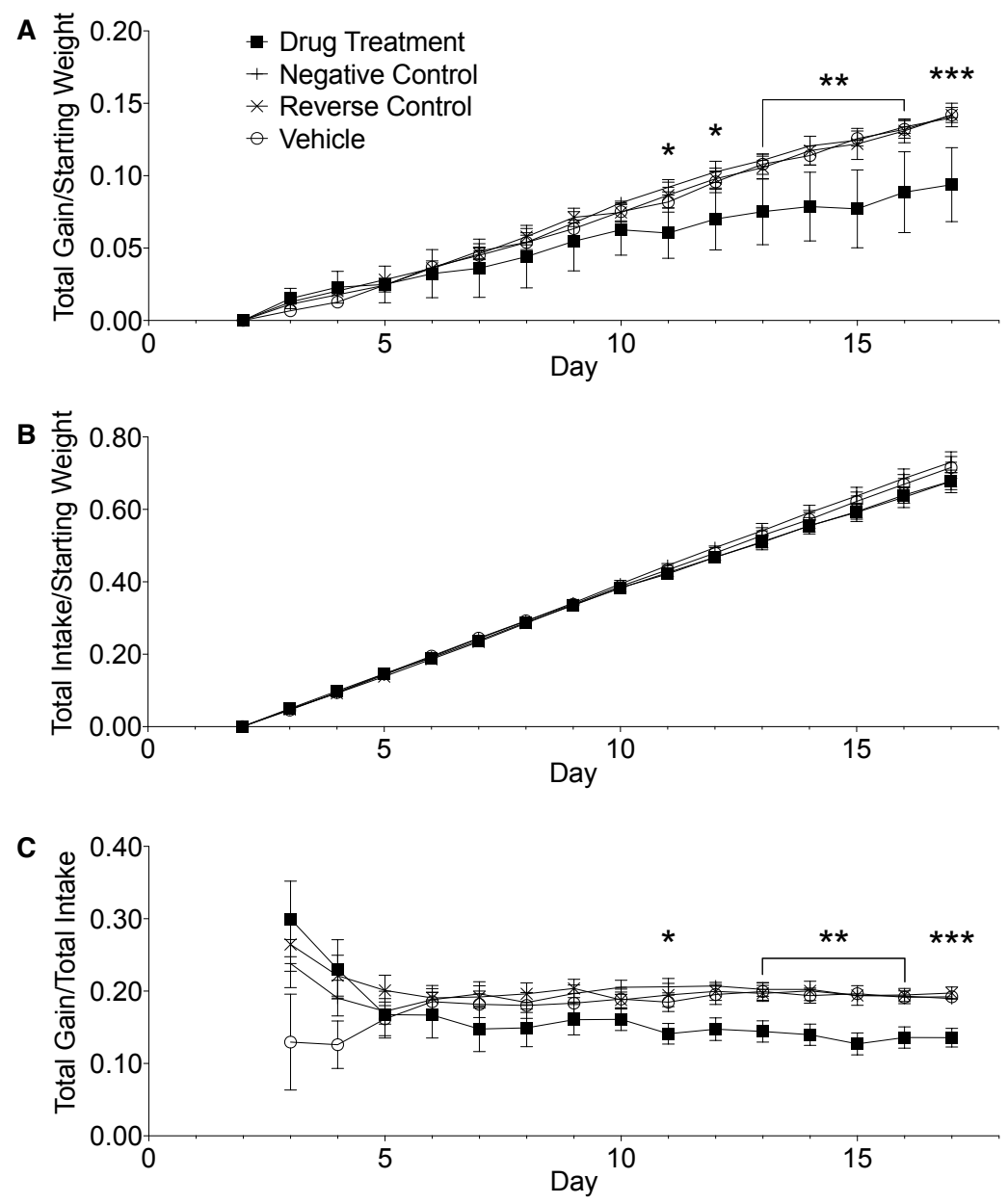

Figure 5.3: Effects of knockdown of central GOAT by anti-sense administered to the lateral ventricles.

Cannula implantation occurred on day 0 , followed by a two-day recovery period. (A) $\mathrm{VMO}$ drug treatment effect on total percentage body weight gain since day 2 (two days after surgery). (B) VMO drug treatment effect on total HFD consumption since day 2, as a fraction of body weight on day 2. (C) VMO drug treatment effect on total body weight gain since day 2 per total HFD consumed since day 2 . All data are mean \pm SEM. ${ }^{*} p<0.05$ drug treatment vs. all control groups; ${ }^{* *} p<0.01$ drug treatment vs. all control groups; ${ }^{* * *} p<0.001$ drug treatment vs. all control groups; by two-way repeated measures ANOVA, one-way ANOVA, and Fisher's LSD post hoc tests. 
LSD post-hocs showed that rats infused with the GOAT antisense gained less weight than those infused with vehicle or control infusates. These differences were statistically significant on days $11-17$ of the treatment period $(p<0.05)$. There were no differences between control groups (Figure 5.3a).

\section{Effect of Drug Treatment on Food Intake}

Repeated measures two-way ANOVA using the Greenhouse-Geisser correction indicated no significant interaction between day and treatment group on total food consumed over starting weight $\left(F_{(3.442,25.241)}=0.988, p=0.423\right)$, indicating that no differences in food consumed over starting weight occurred between treatment groups on any day (Figure 5.3b).

\section{Effect of Drug Treatment on Weight Gain as a Function of Food Intake}

Repeated measures two-way ANOVA using the Greenhouse-Geisser correction indicated a significant interaction between day and treatment group $\left(F_{(8.098,59.382)}=2.786\right.$, $p<0.05$ ), indicating that differences in total weight gain per total food consumed were present between treatment groups on different days. One-way ANOVAs followed by Fisher's LSD post-hocs showed that rats treated with the GOAT antisense gained less weight per food consumed on all days tested. This effect was statistically significant from experimental days 11 to 17 (Day 12 of treatment, $p=0.068$ ). There were no differences between control groups (Figure 5.3c).

\subsection{Discussion}

The purpose of this experiment was two-fold: 1) to verify that GOAT mRNA expression exists in the rat hypothalamus and that the levels of these transcript are modulated by metabolic challenges, and 2) to examine the effect of central GOAT knockdown by vivo-morpholino anti-sense on food intake and weight gain in animals exposed to a high fat diet.

Data from Experiment 1 provide evidence that GOAT mRNA is modulated in the stomach and hypothalamus of rats following metabolic stress. These data supplement previous reports of the presence of GOAT message in the hypothalamus of rodents $(26,137,139)$. After 48 hours of food deprivation, these increases in GOAT transcription occurred in concert with increases in hypothalamic ghrelin mRNA levels. While there is debate on whether ghrelin is synthesized within the hypothalamus (229, $297,300)$, these data certainly support the notion that ghrelin is produced and acylated locally within the hypothalamus particularly in situations of negative energy state. Perhaps more interesting is data from Experiment 2. Here, knockdown of central GOAT mRNA expression using morpholino antisense oligonucleotides decreased weight gain 
and caloric efficiency in rats exposed to a high fat diet. The decrease in weight gain seen in the current experiment is very similar to that seen in GOAT KO mice, or in mice that are treated with GO-CoA-Tat and exposed to diets rich in fatty acids $(233,236)$. In the current study, the lack of difference in caloric intake between knock-down and control rats suggest that the weight loss is not due to reductions in total caloric intake. Due to acylated ghrelin's important role in protecting fat stores $(17,18)$, it is likely that GOAT knockdown animals show reduced body mass due to an increase in the utilization of body fat as a substrate for energy production. When adjusting weight gain per calorie consumed as a measure of metabolism, it is clear that animals treated with the GOAT antisense were gaining less weight per calorie consumed compared to rats in the control groups, again suggesting that central GOAT mRNA expression may play a role in energy balance. While it remains possible that central GOAT acylates ghrelin locally in the hypothalamus, it is also possible that this enzyme modifies other, yet to be determined, proteins that may in themselves be important modulators of food intake and energy balance in the hypothalamus. Supporting additional roles of GOAT beyond that of acylating ghrelin is Sakata and colleague's measurements showing that, of cells expressing GOAT mRNA, 14.5\% of gastric mucosal cells and 19.4\% of duodenal cells do not co-express ghrelin mRNA (26).

Given that the stomach fundus is believed to be the primary source of circulating ghrelin $(10,257)$, it is interesting that we did not find any significant changes in ghrelin mRNA in samples of the fundus of rats fasted for 48 hours or after three weeks of food restriction. This was not completely unexpected as others have found no changes in ghrelin mRNA expression in the stomach following a fast (299). It is possible that food deprivation-induced elevations in plasma acylated ghrelin concentrations may be in fact due to increased GOAT mRNA expression, rather than increased ghrelin expression. This would suggest that a change in the acyl/desacyl ghrelin ratio occurs without changes in denovo ghrelin synthesis. It is also possible that other sources of circulating acylated ghrelin contribute to this increase including ghrelin secreted from other parts of the digestive tract. In addition to the changes in ghrelin and GOAT mRNA, elevations in GHSR1a mRNA were found after 48 hours of food deprivation in the fundus and in response to three weeks of food restriction in the hypothalamus. The role of GHSR1a in the fundus has not been fully determined but the receptor may be an important component in signaling through the gastric vagal afferent pathway $(105,276)$. In addition, ghrelin also has autocrine effects in the stomach to modulate gut motility and to protect against gastric ulceration $(301,302)$. While it is difficult to speculate about the source of the differences in GHSR1a mRNA observed in the stomach versus the hypothalamus, it is quite possible that changes in GHSR1a mRNA expression in the fundus are not associated with energy state, but more with gastro protection during the acute fast, and the result of compensatory down-regulation of GHSR1a mRNA expression following prolonged elevations in circulating acylated ghrelin in the chronic 
food restriction paradigm.

Our results indicating that acylated ghrelin levels are highest with long-term metabolic challenge suggest that the ghrelin-GOAT system may be particularly important for circumstances where undernutrition is prolonged. Indeed, ghrelin has been implicated in the mechanisms through which mammals adapt to under-nutrition including the generation of glucose. Interestingly, both GOAT and GHSR KO mice have difficulty adapting to caloric restriction paradigms and are hypoglycemic, and this state can be reversed in GOAT KO animals by injections of ghrelin or growth hormone $(234,258)$.

In all, our data suggest that the GOAT enzyme is produced in the hypothalamus and it plays a role in regulating energy balance. The mechanisms by which GOAT has these effects remain unclear. One could suggest that these mechanisms involve the acylation of locally produced ghrelin. Alternatively, GOAT may generate these effects independently from ghrelin, yet work is needed to determine if this is the case. 


\section{Chapter 6}

\section{General Discussion}

Using various approaches we have demonstrated the utility of two methods of targeting the ghrelin system to improve phenotypes associated with metabolic disorders. Our first experiment demonstrated that the GOAT inhibitor octanoyl Dap3-ghrelin(1-5) administered subcutaneously and chronically had no effect on either food intake or body weight gain. We suspected that this drug was not capable of penetrating cell membranes to access ER-bound GOAT, although alternate explanations are certainly possible, including problems related to dosage, stability, and route of administration. Despite this, our negative results led us to our second experiment centered around the development of CF801 with the goal of generating a membrane-permeable GOATtargeting drug. In this study, we were able to reduce weight gain in animals given ad lib access to both regular chow and a high-fat diet by interperitoneally administering CF801. Our next step was to examine the possibility of a central role of GOAT, which was motivated in part by previous measurements of GOAT mRNA in the brain. Our use of a VMOs in our final experiment was also based on the requirement of membrane-permeability, albeit with a different purpose of targeting mRNA, along with the increased stability of VMOs when compared to standard anti-sense strands. Using this technique, we were able to, for the first time, demonstrate that GOAT specifically located within the central nervous system has a considerable influence on body mass. By targeting GOAT at the translational level, we were able to reduce weight gain much as we were able to with peripherally administered CF801.

\subsection{Elaborating on the central effects of GOAT}

In the studies presented, we have contributed to the field of ghrelin research in several ways. Of particular importance was our final study demonstrating a critical role of central GOAT and provides a framework for future studies examining the enzyme's 
role in the brain. From here, we can pose further questions that remain to be answered. In particular, it is important to tease apart the individual factors contributing to the reduced weight gain observed in our animals. Our VMO study indicated that this was not a result of reduced food intake. One might suspect that a shift away from glucose utilization towards fat utilization is promoted, based on acylated ghrelin's known role in protecting fat stores $(17,18,67)$. This might involve a reduction in AMPK activity due to reduced ghrelin acylation and a reduction in upstream GHSR signaling. A reduction in AMPK activity would provide a signal of increased energy and lipid availability. This is akin to leptin's inhibitory effect on AMPK in the PVN and arcuate nucleus (111). To address the issue of substrate utilization, measurements of respiratory exchange ratio using metabolic chambers while undergoing VMO treatment can help provide an answer. Additionally, EchoMRI measurements can also help identify if central GOAT knockdown specifically modulates fat mass over lean mass similarly to what was observed with CF801. Comparing the effects of central CF801 to our VMO data may also provide insight into the mechanism of action of our novel drug. Will CF801-treated animals show similar results as VMO-treated animals? If the data do match, it provides support to the notion that similar pathways are being affected. If the data do not match, we must look at alternate explanations and possible comparison to the effect of other drugs, such as AZP-531.

Due to the limited knowledge surrounding central GOAT, we took the broad approach of administering the VMO to the lateral ventricles, which provides access to a considerable number of neuroanatomical regions. Our main interest was areas of the hypothalamus, including the arcuate and PVN, due to their known roles in feeding and metabolism. With our current data, however, we can not narrow down the specific areas involved in the effects that were observed. More directed cannula placements to various regions of the hypothalamus as well as extrahypothalamic areas can help identify the neuroanatomical-specific roles that GOAT plays. This is warranted not only for VMO treatment but also for CF801 treatment. This provides a more directed approach to compare the similarities and differences between the two drugs.

\subsection{Anti-GOAT VMOs, CF801, and desacyl ghrelin}

As discussed previously we do not know the exact mechanism by which CF801 acts to reduce weight gain. Our data indicate that CF801 is at best a weak GOAT inhibitor, despite its ability to reduce circulating acylated ghrelin. In order to examine the possibility that CF801 is acting as a desacyl ghrelin analog, it may be beneficial to examine the known effects of desacyl ghrelin as discussed in section 1.3. These include, among others, inhibition of various effects of co-administered acylated ghrelin, including acylated ghrelin's ability to decrease insulin levels, increase plasma glucose, and increase 
feeding. Replication of these studies using both CF801 and desacyl ghrelin, and comparing if similar results are obtained from both compounds, may help in supporting or refuting the claim that CF801 is acting as a desacyl ghrelin analog. The possibility of a desacyl ghrelin receptor has already been brought up several times. If such a receptor is discovered, validating CF801 as a desacyl ghrelin analog that acts through this receptor can be accomplished by examining the response of a cell line expressing the receptor to CF801 treatment. Until then, we must take a more indirect approach.

Along similar lines, while our VMO targeting GOAT mRNA does not act as a desacyl ghrelin analog, the ultimate effects might be expected to be similar to those of a desacyl ghrelin analog. By reducing GOAT expression we reduce acylation of newlyformed proghrelin. While we did not perform measures of desacyl and acyl ghrelin in our final study examining the effect of VMO treatment, it seems reasonable to suggest that this reduced acylation might also lead to an increase in desacyl ghrelin levels, provided that these unacylated peptides are released from the cell. These measurements can be performed in future studies. Furthermore, despite their differences in central versus peripheral administration, both the VMOs and CF801 had similar effects in terms of weight gain. Given that acylated ghrelin amplifies weight gain whether administered centrally or peripherally, it might also be possible that desacyl ghrelin has similar effects on various parameters, including weight gain, regardless of its method of administration. The different pathways involved in the central and peripheral ghrelin systems still require much characterization.

\subsection{Potential barriers in human translation}

The data presented in this thesis related to both CF801 and our anti-GOAT VMO, along with results from related drugs such as GO-CoA-Tat and AZP-531, provide promising results in relation to drug treatment for metabolic disorders in humans, particularly obesity. There is, however, much more work that must be performed before any human treatment can be considered. In particular, we suspect a wide array of sideeffects for any drug that targets the ghrelin system, whether it be a drug that inhibits GOAT, reduces acylated ghrelin, targets the receptor, or mimics desacyl ghrelin. Some of the roles that ghrelin plays beyond adiposity have already been mentioned. Along with these, dimerization of the ghrelin receptor with a slew of other receptors further demonstrates the complexity of the system and the potential side-effects that may arise due to influences in other pathways. The GHSR has been identified to dimerize with $\mathrm{D}_{1} \mathrm{R}(74)$, dopamine 2 receptor $\left(\mathrm{D}_{2} \mathrm{R}\right)(303)$, 5-hydroxytryptamine $2 \mathrm{C}$ receptor (5HT2C) (304), SST5 (82), and MC3R (305). It is expected that the drugs discussed, while working through different mechanisms, would all in some way influence GHSR signaling, and hence influence dimer partners as well (for review, see (306)). 
In terms of side-effects, one area of particular interest should be the effect of these drugs on stress, anxiety, and depression, given ghrelin's involvement in these three factors (46). A more detailed look at how CF801, anti-GOAT VMOs, GO-CoATat, and AZP-531 affect these variables is warranted. These should include typical behavioral studies such as the elevated plus maze, open field test, forced swim test, and sucrose preference test, along with measurements of HPA axis activity and glucocorticoid levels. Once further data are collected, the best approach to minimizing these side-effects should be examined. Depending on the results, possibilities include co-administration with other drugs such as selective serotonin reuptake inhibitors (SSRIs) and anti-anxiety medication, or perhaps reductions in dosage with addition of low doses of other drugs designed to combat obesity through alternate and additional pathways.

Beyond these side-effects, one must also consider the effectiveness of treatment over prolonged periods of time. Our own animal studies, including those of other labs, generally look at timeframes of approximately two weeks to several months. Given the many redundancies found within metabolic pathways along with the adaptive plasticity discussed previously it is possible that physiological changes would take place to counteract the effects of drug treatment, resulting in tolerance and reducing the effectiveness of treatment. Extending the duration of the experiments conducted in this thesis will help address this issue.

Given the invasiveness of central administration, the ICV infusion of anti-GOAT VMOs is not tenable to human treatment. Peripheral administration, as was done with CF801, is much more feasible. While oral administration is usually desirable, the peptide nature of CF801 removes this possibility. Problems related to stability and bioavailability would arise. This peptide nature, however, along with the lack of any major synthetic modifications, provides an advantage of reducing financial burdens associated with studies, given its relatively low cost of synthesis.

Whether or not any of these drugs can be translated to humans, their utility in scientific research still remains. Despite all the unknowns discussed in this section, preliminary data presented in this thesis certainly support the notion that targeting the ghrelin system through various mechanisms may provide a useful approach to combatting obesity and other metabolic disorders in humans.

\subsection{Conclusion}

Interest in the ghrelin system has risen dramatically since the receptor was identified in 1996 and the peptide discovered in 1999. Its critical role in feeding behavior and it being the first identified peripherally circulating orexigenic peptide has placed ghrelin among the forefront of obesity research. In 2008, the identification of GOAT as the en- 
zyme responsible for acylating ghrelin provided an additional target for research. These discoveries have led to the development of several drugs that target the ghrelin system as well as countless studies characterizing how ghrelin and its counterparts influence feeding, obesity, diabetes, and various other factors important to human health. We have demonstrated here two novel approaches for beneficially targeting this system, including the development and early characterization of our own drug that reduces weight gain, and have also provided new data identifying a role of central GOAT that had not been examined previously. We hope that these discoveries contribute to the current scientific knowledge surrounding ghrelin and the steps required to a better understanding of metabolic disorders and obesity. 
Appendices 


\section{Appendix A}

\section{Copyright Documentation}

\section{A.1 Permission from Frontiers in Endocrinology}

Frontiers copyright policy: "Under the Frontiers Terms and Conditions, authors retain the copyright to their work. All Frontiers articles are Open Access and distributed under the terms of the Creative Commons Attribution License, (CC-BY 4.0), which permits the use, distribution and reproduction of material from published articles, provided the original authors and source are credited, and subject to any copyright notices concerning any third-party content." 


\section{A.2 Permission from Peptides}

\begin{tabular}{ll}
\hline Supplier & Elsevier Limited \\
& The Boulevard, Langford Lane \\
& Kidlington, Oxford, OX5 1GB, UK \\
& 1982084 \\
Registered Company Number & Martin Wellman \\
Customer name & 3818321481477 \\
License number & Feb 29, 2016 \\
License date & Elsevier \\
Licensed content publisher & Peptides \\
Licensed content publication & Knockdown of central ghrelin O- \\
Licensed content title & acyltransferase by vivo-morpholino re- \\
& duces body mass of rats fed a high-fat \\
& diet \\
Licensed content author & Martin Wellman, Alfonso Abizaid \\
Licensed content date & August 2015 \\
Licensed content volume number & 70 \\
Licensed content issue number & $n$ n/a \\
Number of pages & 6 \\
Start Page & 17 \\
End Page & 22 \\
Type of Use & reuse in a thesis/dissertation \\
Portion & full article \\
Format & both print and electronic \\
Are you the author of this Elsevier article? & Yes \\
Will you be translating? & No \\
Title of your thesis/dissertation & The role of ghrelin and ghrelin O- \\
& acyltransferase in obesity: Targets for \\
Expected completion date & treatment \\
Estimated size (number of pages) & May 2016 \\
\hline
\end{tabular}


A.3 Co-author permission statements 
To whom it may concern,

I, Jeffrey M. Zigman, do hereby grant permission to Martin Wellman to reproduce all content associated with "Novel regulator of acylated ghrelin, CF801, reduces weight gain, rebound feeding after a fast, and adiposity in mice" published in Frontiers in Endocrinology.

I am also aware that the author of this thesis will also be granting non-exclusive licenses to Carleton University Library and Library and Archives Canada.

Signature:

Name in print:

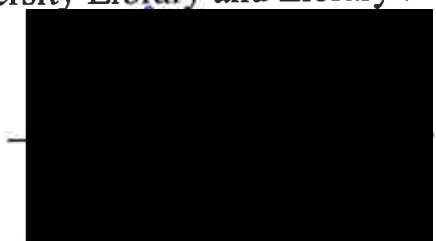

Date: $\quad 2 \cdot 8 \cdot 16$ 
To whom it may concern,

I, Bharath K. Mani, do hereby grant permission to Martin Wellman to reproduce all content associated with "Novel regulator of acylated ghrelin, CF801, reduces weight gain, rebound feeding after a fast, and adiposity in mice" published in Frontiers in Endocrinology.

I am also aware that the author of this thesis will also be granting non-exclusive licenses to Carleton University Library and Library and Archives Canada.

Signature:

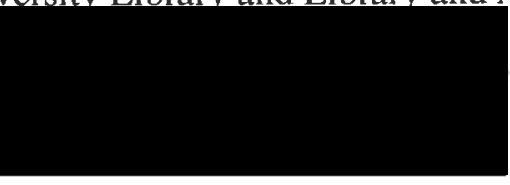

Name in print: Bharath K. Mani

Date:

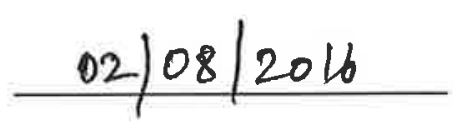


To whom it may concern,

I, Harry MacKay, do hereby grant permission to Martin Wellman to reproduce all content associated with "Novel regulator of acylated ghrelin, CF801, reduces weight gain, rebound feeding after a fast, and adiposity in mice" published in Frontiers in Endocrinology.

I am also aware that the author of this thesis will also be granting non-exclusive licenses to Carleton University Library and Library and Archives Canada.

Signature:

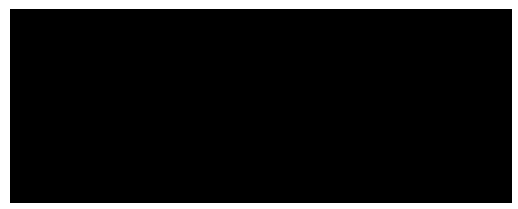

Name in print: Harry MacKay

Date:

February 8, 2016 
To whom it may concern,

I, Joseph E. Darling, do hereby grant permission to Martin Wellman to reproduce all content associated with "Novel regulator of acylated ghrelin, CF801, reduces weight gain, rebound feeding after a fast, and adiposity in mice" published in Frontiers in Endocrinology.

I am also aware that the author of this thesis will also be granting non-exclusive licenses to Carleton University Library and Library and Archives Canada.

Signature:

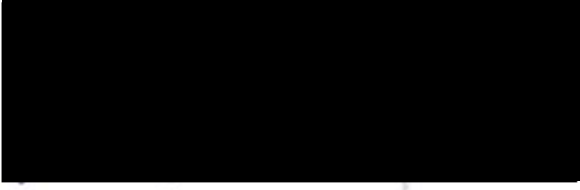

Name in print: Joseph E. Darling

Date:

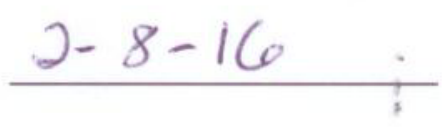


To whom it may concern,

I, James L. Hougland, do hereby grant permission to Martin Wellman to reproduce all content associated with "Novel regulator of acylated ghrelin, CF801, reduces weight gain, rebound feeding after a fast, and adiposity in mice" published in Frontiers in Endocrinology.

I am also aware that the author of this thesis will also be granting non-exclusive licenses to Carleton University Librarv and_žbrary and Archives Canada.

Signature:

Name in print: James L. Hougland

Date:

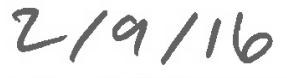


To whom it may concern,

I, Alfonso Abizaid, do hereby grant permission to Martin Wellman to reproduce all content associated with "Novel regulator of acylated ghrelin, CF801, reduces weight gain, rebound feeding after a fast, and adiposity in mice" published in Frontiers in Endocrinology.

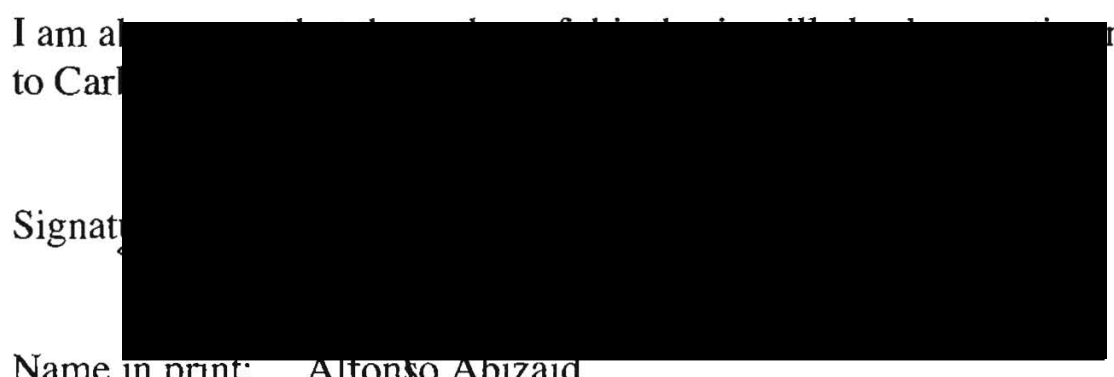

non-exclusive licenses

Name in print: Altonso Abizald

Date:

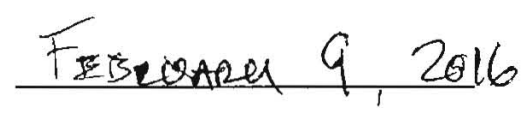


To whom it may concern,

I, Alfonso Abizaid, do hereby grant permission to Martin Wellman to reproduce all content associated with "Knockdown of central ghrelin $\mathrm{O}$-acyltransferase by vivomorpholino reduces body mass of rats fed a high-fat diet" published in Peptides.

I am also aware that the author of this thesis will also be granting non-exclusive licenses

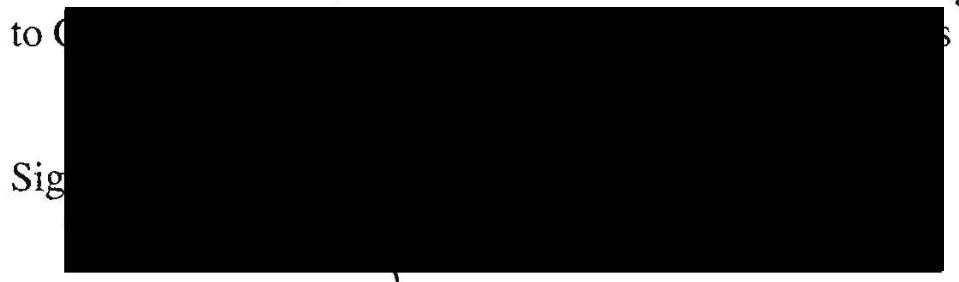

Name in print: Alfonso Abizaid

Date:

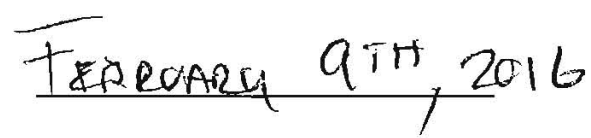




\section{Bibliography}

(1) World Health Organization Fact sheet N³11: Obesity and overweight., 2015.

(2) Thompson, D., Edelsberg, J., Colditz, G. A., Bird, A. P., and Oster, G. (1999). LIfetime health and economic consequences of obesity. Archives of Internal Medicine 159, 2177-2183.

(3) Kopelman, P. G. (2000). Obesity as a medical problem. Nature 404, 635-643.

(4) Friedman, J. M. (2000). Obesity in the new millennium. Nature 404, 632634.

(5) Abizaid, A., and Horvath, T. L. (2006). Rimonabant-a new hope in the treatment of obesity? Nat Clin Pract End Met 2, 370-371.

(6) Christensen, R., Kristensen, P. K., Bartels, E. M., Bliddal, H., and Astrup, A. (2007). Efficacy and safety of the weight-loss drug rimonabant: a meta-analysis of randomised trials. The Lancet 370, 1706-1713.

(7) Padwal, R. S., and Majumdar, S. R. (2007). Drug treatments for obesity: orlistat, sibutramine, and rimonabant. The Lancet 369, 71-77.

(8) Rumsfeld, J. S., and Nallamothu, B. K. (2008). The hope and fear of rimonabant. JAMA 299, 1601-1602.

(9) Kojima, M., Hosoda, H., Date, Y., Nakazato, M., Matsuo, H., and Kangawa, K. (1999). Ghrelin is a growth-hormone-releasing acylated peptide from stomach. Nature 402, 656-660.

(10) Date, Y., Kojima, M., Hosoda, H., Sawaguchi, A., Mondal, M. S., Suganuma, T., Matsukura, S., Kangawa, K., and Nakazato, M. (2000). Ghrelin, a Novel Growth Hormone-Releasing Acylated Peptide, Is Synthesized in a Distinct Endocrine Cell Type in the Gastrointestinal Tracts of Rats and Humans. Endocrinology 141, 4255-4261.

(11) Hosoda, H., Kojima, M., Matsuo, H., and Kangawa, K. (2000). Ghrelin and Des-acyl Ghrelin: Two Major Forms of Rat Ghrelin Peptide in Gastrointestinal Tissue. Biochemical and Biophysical Research Communications 279, 909-913. 
(12) Korbonits, M., Bustin, S. A., Kojima, M., Jordan, S., Adams, E. F., Lowe, D. G., Kangawa, K., and Grossman, A. B. (2001). The Expression of the Growth Hormone Secretagogue Receptor Ligand Ghrelin in Normal and Abnormal Human Pituitary and Other Neuroendocrine Tumors. The Journal of Clinical Endocrinology \& Metabolism 86, 881-887.

(13) Ariyasu, H., Takaya, K., Tagami, T., Ogawa, Y., Hosoda, K., Akamizu, T., Suda, M., Koh, T., Natsui, K., Toyooka, S., Shirakami, G., Usui, T., Shimatsu, A., Doi, K., Hosoda, H., Kojima, M., Kangawa, K., and Nakao, K. (2001). Stomach Is a Major Source of Circulating Ghrelin, and Feeding State Determines Plasma Ghrelin-Like Immunoreactivity Levels in Humans. The Journal of Clinical Endocrinology \& Metabolism 86, 4753-4758.

(14) Sakata, I., Nakamura, K., Yamazaki, M., Matsubara, M., Hayashi, Y., Kangawa, K., and Sakai, T. (2002). Ghrelin-producing cells exist as two types of cells, closed- and opened-type cells, in the rat gastrointestinal tract. Peptides 23, 531-536.

(15) Gnanapavan, S., Kola, B., Bustin, S. A., Morris, D. G., McGee, P., Fairclough, P., Bhattacharya, S., Carpenter, R., Grossman, A. B., and Korbonits, M. (2002). The Tissue Distribution of the mRNA of Ghrelin and Subtypes of Its Receptor, GHS-R, in Humans. The Journal of Clinical Endocrinology \& Metabolism 87, 2988.

(16) Ghelardoni, S., Carnicelli, V., Frascarelli, S., Ronca-Testoni, S., and Zucchi, R. (2006). Ghrelin tissue distribution: Comparison between gene and protein expression., English Journal of Endocrinological Investigation 29, 115-121.

(17) Tschöp, M., Smiley, D. L., and Heiman, M. L. (2000). Ghrelin induces adiposity in rodents. Nature 407, 908-913.

(18) Zigman, J. M., Nakano, Y., Coppari, R., Balthasar, N., Marcus, J. N., Lee, C. E., Jones, J. E., Deysher, A. E., Waxman, A. R., White, R. D., Williams, T. D., Lachey, J. L., Seeley, R. J., Lowell, B. B., and Elmquist, J. K. (2005). Mice lacking ghrelin receptors resist the development of diet-induced obesity. The Journal of Clinical Investigation 115, 3564-3572.

(19) Gutierrez, J. A., Solenberg, P. J., Perkins, D. R., Willency, J. A., Knierman, M. D., Jin, Z., Witcher, D. R., Luo, S., Onyia, J. E., and Hale, J. E. (2008). Ghrelin octanoylation mediated by an orphan lipid transferase. Proceedings of the National Academy of Sciences 105, 6320-6325.

(20) Yang, J., Brown, M. S., Liang, G., Grishin, N. V., and Goldstein, J. L. (2008). Identification of the Acyltransferase that Octanoylates Ghrelin, an AppetiteStimulating Peptide Hormone. Cell 132, 387-396. 
(21) Yoshimoto, A., Mori, K., Sugawara, A., Mukoyama, M., Yahata, K., Suganami, T., Takaya, K., Hosoda, H., Kojima, M., Kangawa, K., and Nakao, K. (2002). Plasma Ghrelin and Desacyl Ghrelin Concentrations in Renal Failure. Journal of the American Society of Nephrology 13, 2748-2752.

(22) Patterson, M., Murphy, K. G., le Roux, C. W., Ghatei, M. A., and Bloom, S. R. (2005). Characterization of Ghrelin-Like Immunoreactivity in Human Plasma. The Journal of Clinical Endocrinology \& Metabolism 90, 2205-2211.

(23) Gómez, R., Lago, F., Gómez-Reino, J. J., Dieguez, C., and Gualillo, O. (2009). Expression and modulation of ghrelin O-acyltransferase in cultured chondrocytes. Arthritis and Rheumatism 60, 1704-1709.

(24) González, C. R., Vázquez, M. J., López, M., and Diéguez, C. (2008). Influence of chronic undernutrition and leptin on GOAT mRNA levels in rat stomach mucosa. Journal of Molecular Endocrinology 41, 415-421.

(25) Matsumoto, M., Hosoda, H., Kitajima, Y., Morozumi, N., Minamitake, Y., Tanaka, S., Matsuo, H., Kojima, M., Hayashi, Y., and Kangawa, K. (2001). Structure-Activity Relationship of Ghrelin: Pharmacological Study of Ghrelin Peptides. Biochemical and Biophysical Research Communications 287, 142-146.

(26) Sakata, I., Yang, J., Lee, C. E., Osborne-Lawrence, S., Rovinsky, S. A., Elmquist, J. K., and Zigman, J. M. (2009). Colocalization of ghrelin $\mathrm{O}$-acyltransferase and ghrelin in gastric mucosal cells. American Journal of Physiology - Endocrinology and Metabolism 297, E134-E141.

(27) Akamizu, T., Takaya, K., Irako, T., Hosoda, H., Teramukai, S., Matsuyama, A., Tada, H., Miura, K., Shimizu, A., Fukushima, M., Yokode, M., Tanaka, K., and Kangawa, K. (2004). Pharmacokinetics, safety, and endocrine and appetite effects of ghrelin administration in young healthy subjects. European Journal of Endocrinology 150, 447-455.

(28) Hosoda, H., and Kangawa, K. In Methods in Enzymology, Masayasu, K., and Kenji, K., Eds.; Academic Press: 2012; Vol. Volume 514, pp 113-126.

(29) Colombo, M., Gregersen, S., Xiao, J., and Hermansen, K. (2003). Effects of Ghrelin and Other Neuropeptides (CART, MCH, Orexin A and B, and GLP1) on the Release of Insulin From Isolated Rat Islets. Pancreas 27, 161-166.

(30) Dezaki, K., Hosoda, H., Kakei, M., Hashiguchi, S., Watanabe, M., Kangawa, K., and Yada, T. (2004). Endogenous Ghrelin in Pancreatic Islets Restricts Insulin Release by Attenuating Ca2+ Signaling in $\beta$-Cells: Implication in the Glycemic Control in Rodents. Diabetes 53, 3142-3151. 
(31) Dezaki, K., Sone, H., Koizumi, M., Nakata, M., Kakei, M., Nagai, H., Hosoda, H., Kangawa, K., and Yada, T. (2006). Blockade of Pancreatic Islet-Derived Ghrelin Enhances Insulin Secretion to Prevent High-Fat Diet-Induced Glucose Intolerance. Diabetes 55, 3486-3493.

(32) Doi, A., Shono, T., Nishi, M., Furuta, H., Sasaki, H., and Nanjo, K. (2006). IA- $2 \beta$, but not IA-2, is induced by ghrelin and inhibits glucose-stimulated insulin secretion. Proceedings of the National Academy of Sciences of the United States of America 103, 885-890.

(33) Egido, E. M., Rodriguez-Gallardo, J., Silvestre, R. A., and Marco, J. (2002). Inhibitory effect of ghrelin on insulin and pancreatic somatostatin secretion. European Journal of Endocrinology 146, 241-244.

(34) Reimer, M. K., Pacini, G., and Ahrén, B. (2003). Dose-Dependent Inhibition by Ghrelin of Insulin Secretion in the Mouse. Endocrinology 144, 916-921.

(35) Tong, J., Prigeon, R. L., Davis, H. W., Bidlingmaier, M., Kahn, S. E., Cummings, D. E., Tschöp, M. H., and D’Alessio, D. (2010). Ghrelin Suppresses Glucose-Stimulated Insulin Secretion and Deteriorates Glucose Tolerance in Healthy Humans. Diabetes 59, 2145-2151.

(36) Wierup, N., Yang, S., McEvilly, R. J., Mulder, H., and Sundler, F. (2004). Ghrelin Is Expressed in a Novel Endocrine Cell Type in Developing Rat Islets and Inhibits Insulin Secretion from INS-1 (832/13) Cells. Journal of Histochemistry \& Cytochemistry 52, 301-310.

(37) Date, Y., Nakazato, M., Murakami, N., Kojima, M., Kangawa, K., and Matsukura, S. (2001). Ghrelin Acts in the Central Nervous System to Stimulate Gastric Acid Secretion. Biochemical and Biophysical Research Communications 280, 904-907.

(38) Ejskjaer, N., Wo, J. M., Esfandyari, T., Mazen Jamal, M., Dimcevski, G., Tarnow, L., Malik, R. A., Hellström, P. M., Mondou, E., Quinn, J., Rousseau, F., and McCallum, R. W. (2013). A phase 2a, randomized, double-blind 28day study of TZP-102 a ghrelin receptor agonist for diabetic gastroparesis. Neurogastroenterology \& Motility 25, e140-e150.

(39) Masuda, Y., Tanaka, T., Inomata, N., Ohnuma, N., Tanaka, S., Itoh, Z., Hosoda, H., Kojima, M., and Kangawa, K. (2000). Ghrelin Stimulates Gastric Acid Secretion and Motility in Rats. Biochemical and Biophysical Research Communications 276, 905-908.

(40) Trudel, L., Tomasetto, C., Rio, M. C., Bouin, M., Plourde, V., Eberling, P., and Poitras, P., Ghrelin/motilin-related peptide is a potent prokinetic to reverse gastric postoperative ileus in rat; 6, 2002; Vol. 282, G948-G952. 
(41) Asakawa, A., Inui, A., Kaga, T., Yuzuriha, H., Nagata, T., Fujimiya, M., Katsuura, G., Makino, S., Fujino, M. A., and Kasuga, M. (2001). A Role of Ghrelin in Neuroendocrine and Behavioral Responses to Stress in Mice. Neuroendocrinology 74, 143-147.

(42) Carlini, V. P., Monzón, M. E., Varas, M. M., Cragnolini, A. B., Schiöth, H. B., Scimonelli, T. N., and de Barioglio, S. R. (2002). Ghrelin increases anxiety-like behavior and memory retention in rats. Biochemical and Biophysical Research Communications 299, 739-743.

(43) Carlini, V. P., Varas, M. M., Cragnolini, A. B., Schiöth, H. B., Scimonelli, T. N., and de Barioglio, S. R. (2004). Differential role of the hippocampus, amygdala, and dorsal raphe nucleus in regulating feeding, memory, and anxiety-like behavioral responses to ghrelin. Biochemical and Biophysical Research Communications 313, 635-641.

(44) Kanehisa, M., Akiyoshi, J., Kitaichi, T., Matsushita, H., Tanaka, E., Kodama, K., Hanada, H., and Isogawa, K. (2006). Administration of antisense DNA for ghrelin causes an antidepressant and anxiolytic response in rats. Progress in Neuro-Psychopharmacology and Biological Psychiatry 30, 1403-1407.

(45) Kristenssson, E., Sundqvist, M., Astin, M., Kjerling, M., Mattsson, H., Dornonville de la Cour, C., Håkanson, R., and Lindström, E. (2006). Acute psychological stress raises plasma ghrelin in the rat. Regulatory Peptides 134, 114-117.

(46) Lutter, M., Sakata, I., Osborne-Lawrence, S., Rovinsky, S. A., Anderson, J. G., Jung, S., Birnbaum, S., Yanagisawa, M., Elmquist, J. K., Nestler, E. J., and Zigman, J. M. (2008). The orexigenic hormone ghrelin defends against depressive symptoms of chronic stress. Nat Neurosci 11, 752-753.

(47) Carvajal, P., Carlini, V. P., Schiöth, H. B., de Barioglio, S. R., and Salvatierra, N. A. (2009). Central ghrelin increases anxiety in the Open Field test and impairs retention memory in a passive avoidance task in neonatal chicks. Neurobiology of Learning and Memory 91, 402-407.

(48) Baldanzi, G., Filigheddu, N., Cutrupi, S., Catapano, F., Bonissoni, S., Fubini, A., Malan, D., Baj, G., Granata, R., Broglio, F., Papotti, M., Surico, N., Bussolino, F., Isgaard, J., Deghenghi, R., Sinigaglia, F., Prat, M., Muccioli, G., Ghigo, E., and Graziani, A. (2002). Ghrelin and des-acyl ghrelin inhibit cell death in cardiomyocytes and endothelial cells through ERK1/2 and PI 3-kinase/AKT. The Journal of Cell Biology 159, 1029-1037. 
(49) Nagaya, N., Uematsu, M., Kojima, M., Date, Y., Nakazato, M., Okumura, H., Hosoda, H., Shimizu, W., Yamagishi, M., Oya, H., Koh, H., Yutani, C., and Kangawa, K. (2001). Elevated Circulating Level of Ghrelin in Cachexia Associated With Chronic Heart Failure: Relationships Between Ghrelin and Anabolic/Catabolic Factors. Circulation 104, 2034-2038.

(50) Okumura, H., Nagaya, N., Enomoto, M., Nakagawa, E., Oya, H., and Kangawa, K. (2002). Vasodilatory Effect of Ghrelin, an Endogenous Peptide From the Stomach. Journal of Cardiovascular Pharmacology 39, 779-783.

(51) Schwenke, D. O., Tokudome, T., Kishimoto, I., Horio, T., Shirai, M., Cragg, P. A., and Kangawa, K. (2008). Early Ghrelin Treatment after Myocardial Infarction Prevents an Increase in Cardiac Sympathetic Tone and Reduces Mortality. Endocrinology 149, 5172-5176.

(52) Weikel, J. C., Wichniak, A., Ising, M., Brunner, H., Friess, E., Held, K., Mathias, S., Schmid, D. A., Uhr, M., and Steiger, A., Ghrelin promotes slow-wave sleep in humans; 2, 2003; Vol. 284, E407-E415.

(53) Andreis, P. G., Malendowicz, L. K., Trejter, M., Neri, G., Spinazzi, R., Rossi, G. P., and Nussdorfer, G. G. (2003). Ghrelin and growth hormone secretagogue receptor are expressed in the rat adrenal cortex: evidence that ghrelin stimulates the growth, but not the secretory activity of adrenal cells. FEBS Letters 536, 173-179.

(54) Li, R. L., Sherbet, D. P., Elsbernd, B. L., Goldstein, J. L., Brown, M. S., and Zhao, T.-J. (2012). Profound Hypoglycemia in Starved, Ghrelin-deficient Mice Is Caused by Decreased Gluconeogenesis and Reversed by Lactate or Fatty Acids. Journal of Biological Chemistry 287, 17942-17950.

(55) Yi, C.-X., Heppner, K. M., Kirchner, H., Tong, J., Bielohuby, M., Gaylinn, B. D., Müller, T. D., Bartley, E., Davis, H. W., Zhao, Y., Joseph, A., Kruthaupt, T., Ottaway, N., Kabra, D., Habegger, K. M., Benoit, S. C., Bidlingmaier, M., Thorner, M. O., Perez-Tilve, D., Tschöp, M. H., and Pfluger, P. T. (2012). The GOAT-Ghrelin System Is Not Essential for Hypoglycemia Prevention during Prolonged Calorie Restriction. PLoS ONE 7, e32100.

(56) Koo, G. C., Huang, C., Camacho, R., Trainor, C., Blake, J. T., SirotinaMeisher, a., Schleim, K. D., Wu, T. J., Cheng, K., Nargund, R., and McKissick, G. (2001). Immune enhancing effect of a growth hormone secretagogue. Journal of immunology (Baltimore, Md. : 1950) 166, 41954201.

(57) Cummings, D. E. (2006). Ghrelin and the short- and long-term regulation of appetite and body weight. Physiology \& Behavior 89, 71-84. 
(58) Cummings, D. E., Purnell, J. Q., Frayo, R. S., Schmidova, K., Wisse, B. E., and Weigle, D. S. (2001). A Preprandial Rise in Plasma Ghrelin Levels Suggests a Role in Meal Initiation in Humans. Diabetes 50, 1714-1719.

(59) Tschöp, M., Wawarta, R., Riepl, R. L., Friedrich, S., Bidlingmaier, M., Landgraf, R., and Folwaczny, C. (2001). Post-prandial decrease of circulating human ghrelin levels. Journal of Endocrinological Investigation 24, RC19-21.

(60) Wren, A. M., Small, C. J., Abbott, C. R., Dhillo, W. S., Seal, L. J., Cohen, M. A., Batterham, R. L., Taheri, S., Stanley, S. A., Ghatei, M. A., and Bloom, S. R. (2001). Ghrelin Causes Hyperphagia and Obesity in Rats. Diabetes 50, 2540-2547.

(61) Wren, A. M., Small, C. J., Ward, H. L., Murphy, K. G., Dakin, C. L., Taheri, S., Kennedy, A. R., Roberts, G. H., Morgan, D. G. A., Ghatei, M. A., and Bloom, S. R. (2000). The Novel Hypothalamic Peptide Ghrelin Stimulates Food Intake and Growth Hormone Secretion. Endocrinology 141, 4325-4328.

(62) Wren, A. M., Seal, L. J., Cohen, M. A., Brynes, A. E., Frost, G. S., Murphy, K. G., Dhillo, W. S., Ghatei, M. A., and Bloom, S. R. (2001). Ghrelin Enhances Appetite and Increases Food Intake in Humans. The Journal of Clinical Endocrinology \& Metabolism 86, 5992.

(63) Kamegai, J., Tamura, H., Shimizu, T., Ishii, S., Sugihara, H., and Wakabayashi, I. (2001). Chronic Central Infusion of Ghrelin Increases Hypothalamic Neuropeptide Y and Agouti-Related Protein mRNA Levels and Body Weight in Rats. Diabetes 50, 2438-2443.

(64) Nakazato, M., Murakami, N., Date, Y., Kojima, M., Matsuo, H., Kangawa, K., and Matsukura, S. (2001). A role for ghrelin in the central regulation of feeding. Nature 409, 194-198.

(65) Lawrence, C. B., Snape, A. C., Baudoin, F. M. H., and Luckman, S. M. (2002). Acute central ghrelin and GH secretagogues induce feeding and activate brain appetite centers. Endocrinology 143, 155-162.

(66) Shintani, M., Ogawa, Y., Ebihara, K., Aizawa-abe, M., Miyanaga, F., Takaya, K., Hayashi, T., Inoue, G., Hosoda, K., Kojima, M., Kangawa, K., and Nakao, K. (2001). That Antagonizes Leptin Action Through the Activation of Hypothalamic Neuropeptide Y / Y1 Receptor Pathway. Diabetes 50, 227-232.

(67) Theander-Carrillo, C., Wiedmer, P., Cettour-Rose, P., Nogueiras, R., PerezTilve, D., Pfluger, P., Castaneda, T. R., Muzzin, P., Schürmann, A., Szanto, I., Tschöp, M. H., and Rohner-Jeanrenaud, F. (2006). Ghrelin action in the brain controls adipocyte metabolism. Journal of Clinical Investigation 116, 19831993. 
(68) Shimbara, T., Mondal, M. S., Kawagoe, T., Toshinai, K., Koda, S., Yamaguchi, H., Date, Y., and Nakazato, M. (2004). Central administration of ghrelin preferentially enhances fat ingestion. Neuroscience Letters 369, 75-79.

(69) Perello, M., Sakata, I., Birnbaum, S., Chuang, J. C., Osborne-Lawrence, S., Rovinsky, S. a., Woloszyn, J., Yanagisawa, M., Lutter, M., and Zigman, J. M. (2010). Ghrelin Increases the Rewarding Value of High-Fat Diet in an OrexinDependent Manner. Biological Psychiatry 67, 880-886.

(70) Dallman, M. F., Pecoraro, N., Akana, S. F., la Fleur, S. E., Gomez, F., Houshyar, H., Bell, M. E., Bhatnagar, S., Laugero, K. D., and Manalo, S. (2003). Chronic stress and obesity: A new view of "comfort food". Proceedings of the National Academy of Sciences 100, 11696-11701.

(71) Egecioglu, E., Jerlhag, E., Salomé, N., Skibicka, K. P., Haage, D., Bohlooly-Y, M., Andersson, D., Bjursell, M., Perrissoud, D., Engel, J. A., and Dickson, S. L. (2010). Ghrelin increases intake of rewarding food in rodents. Addiction Biology 15, 304-311.

(72) Chuang, J. C., Perello, M., Sakata, I., Osborne-Lawrence, S., Savitt, J. M., Lutter, M., and Zigman, J. M. (2011). Ghrelin mediates stress-induced foodreward behavior in mice. Journal of Clinical Investigation 121, 2684-2692.

(73) Abizaid, A., Liu, Z. W., Andrews, Z. B., Shanabrough, M., Borok, E., Elsworth, J. D., Roth, R. H., Sleeman, M. W., Picciotto, M. R., Tschöp, M. H., Gao, X. B., and Horvath, T. L. (2006). Ghrelin modulates the activity and synaptic input organization of midbrain dopamine neurons while promoting appetite. Journal of Clinical Investigation 116, 3229-3239.

(74) Jiang, H., Betancourt, L., and Smith, R. G. (2006). Ghrelin amplifies dopamine signaling by cross talk involving formation of growth hormone secretagogue receptor/dopamine receptor subtype 1 heterodimers. Molecular endocrinology (Baltimore, Md.) 20, 1772-1785.

(75) World Health Organization Fact sheet N³12: Diabetes., 2015.

(76) Morrish, N. J., Wang, S. L., Stevens, L. K., Fuller, J. H., and Keen, H. (2001). Mortality and causes of death in the WHO Multinational Study of Vascular Disease in Diabetes. Diabetologia 44 Suppl 2, S14-S21.

(77) Guan, X.-M., YuThese authors contributed equally to this work., H., PalyhaThese authors contributed equally to this work., O. C., McKee, K. K., Feighner, S. D., Sirinathsinghji, D. J. S., Smith, R. G., Van der Ploeg, L. H. T., and Howard, A. D. (1997). Distribution of mRNA encoding the growth hormone secretagogue receptor in brain and peripheral tissues. Molecular Brain Research $48,23-29$. 
(78) Date, Y., Nakazato, M., Hashiguchi, S., Dezaki, K., Mondal, M. S., Hosoda, H., Kojima, M., Kangawa, K., Arima, T., Matsuo, H., Yada, T., and Matsukura, S. (2002). Ghrelin is present in pancreatic $\alpha$-cells of humans and rats and stimulates insulin secretion. Diabetes 51, 124-129.

(79) Broglio, F., Arvat, E., Benso, A., Gottero, C., Muccioli, G., Papotti, M., van der Lely, A. J., Deghenghi, R., and Ghigo, E. (2001). Ghrelin, a Natural GH Secretagogue Produced by the Stomach, Induces Hyperglycemia and Reduces Insulin Secretion in Humans. The Journal of Clinical Endocrinology \& Metabolism 86, 5083.

(80) Broglio, F., Gottero, C., Benso, a., Prodam, F., Destefanis, S., Gauna, C., Maccario, M., Deghenghi, R., Van Der Lely, a. J., and Ghigo, E. (2003). Effects of ghrelin on the insulin and glycemic responses to glucose, arginine, or free fatty acids load in humans. Journal of Clinical Endocrinology and Metabolism $88,4268-4272$.

(81) Tassone, F., Broglio, F., Destefanis, S., Rovere, S., Benso, a., Gottero, C., Prodam, F., Rossetto, R., Gauna, C., van der Lely, a. J., Ghigo, E., and Maccario, M. (2003). Neuroendocrine and metabolic effects of acute ghrelin administration in human obesity. The Journal of clinical endocrinology and metabolism $88,5478-5483$.

(82) Park, S., Jiang, H., Zhang, H., and Smith, R. G. (2012). Modification of ghrelin receptor signaling by somatostatin receptor-5 regulates insulin release. Proceedings of the National Academy of Sciences 109, 19003-19008.

(83) Wang, Q., Liu, C., Uchida, A., Chuang, J.-C., Walker, A., Liu, T., OsborneLawrence, S., Mason, B. L., Mosher, C., Berglund, E. D., Elmquist, J. K., and Zigman, J. M. (2014). Arcuate AgRP neurons mediate orexigenic and glucoregulatory actions of ghrelin. Molecular Metabolism 3, 64-72.

(84) Broglio, F., Gottero, C., Prodam, F., Gauna, C., Muccioli, G., Papotti, M., Abribat, T., Van Der Lely, a. J., and Ghigo, E. (2004). Non-Acylated Ghrelin Counteracts the Metabolic But Not the Neuroendocrine Response to Acylated Ghrelin in Humans. The Journal of Clinical Endocrinology \& Metabolism 89, 3062-3065.

(85) Kumar, R., Salehi, A., Rehfeld, J. F., Höglund, P., Lindström, E., and Håkanson, R. (2010). Proghrelin peptides: Desacyl ghrelin is a powerful inhibitor of acylated ghrelin, likely to impair physiological effects of acyl ghrelin but not of obestatin: A study of pancreatic polypeptide secretion from mouse islets. Regulatory Peptides 164, 65-70. 
(86) Inhoff, T., Mönnikes, H., Noetzel, S., Stengel, A., Goebel, M., Dinh, Q. T., Riedl, A., Bannert, N., Wisser, A.-S., Wiedenmann, B., Klapp, B. F., Taché, Y., and Kobelt, P. (2008). Desacyl ghrelin inhibits the orexigenic effect of peripherally injected ghrelin in rats. Peptides 29, 2159-2168.

(87) Asakawa, A., Inui, A., Fujimiya, M., Sakamaki, R., Shinfuku, N., Ueta, Y., Meguid, M. M., and Kasuga, M. (2005). Stomach regulates energy balance via acylated ghrelin and desacyl ghrelin. Gut 54, 18-24.

(88) Gauna, C., Meyler, F. M., Janssen, J. A. M. J. L., Delhanty, P. J. D., Abribat, T., van Koetsveld, P., Hofland, L. J., Broglio, F., Ghigo, E., and van der Lely, A. J. (2004). Administration of Acylated Ghrelin Reduces Insulin Sensitivity, Whereas the Combination of Acylated Plus Unacylated Ghrelin Strongly Improves Insulin Sensitivity. The Journal of Clinical Endocrinology \& Metabolism 89, 5035-5042.

(89) Gauna, C., Delhanty, P. J. D., Hofland, L. J., Janssen, J. A. M. J. L., Broglio, F., Ross, R. J. M., Ghigo, E., and van der Lely, A. J. (2005). Ghrelin Stimulates, Whereas Des-Octanoyl Ghrelin Inhibits, Glucose Output by Primary Hepatocytes. The Journal of Clinical Endocrinology \& Metabolism 90, 10551060 .

(90) Özcan, B., Neggers, S. J. C. M. M., Miller, A. R., Yang, H.-C., Lucaites, V., Abribat, T., Allas, S., Huisman, M., Visser, J. A., Themmen, A. P. N., Sijbrands, E. J. G., Delhanty, P. J. D., and van der Lely, A. J. (2014). Does desacyl ghrelin improve glycemic control in obese diabetic subjects by decreasing acylated ghrelin levels? European Journal of Endocrinology 170, 799-807.

(91) Zhang, W., Chai, B., Li, J.-y., Wang, H., and Mulholland, M. W. (2008). Effect of Des-acyl Ghrelin on Adiposity and Glucose Metabolism. Endocrinology 149, 4710-4716.

(92) Benso, A., St-Pierre, D. H., Prodam, F., Gramaglia, E., Granata, R., van der Lely, A. J., Ghigo, E., and Broglio, F. (2012). Metabolic effects of overnight continuous infusion of unacylated ghrelin in humans. European Journal of Endocrinology 166, 911-916.

(93) Kleinz, M. J., Maguire, J. J., Skepper, J. N., and Davenport, A. P. (2006). Functional and immunocytochemical evidence for a role of ghrelin and desoctanoyl ghrelin in the regulation of vascular tone in man. Cardiovascular Research 69, 227-235.

(94) Andrews, Z. B. (2011). Central mechanisms involved in the orexigenic actions of ghrelin. Peptides 32, 2248-2255. 
(95) Cowley, M. A., Smith, R. G., Diano, S., Tsch^p, M., Pronchuk, N., Grove, K. L., Strasburger, C. J., Bidlingmaier, M., Esterman, M., Heiman, M. L., Garcia-Segura, L. M., Nillni, E. A., Mendez, P., Low, M. J., Sotonyi, P., Friedman, J. M., Liu, H., Pinto, S., Colmers, W. F., Cone, R. D., and Horvath, T. L. (2003). The Distribution and Mechanism of Action of Ghrelin in the CNS Demonstrates a Novel Hypothalamic Circuit Regulating Energy Homeostasis. Neuron 37, 649-661.

(96) Kamegai, J., Tamura, H., Shimizu, T., Ishii, S., Sugihara, H., and Wakabayashi, I. (2000). Central Effect of Ghrelin, an Endogenous Growth Hormone Secretagogue, on Hypothalamic Peptide Gene Expression. Endocrinology 141, 4797-4800.

(97) Lu, S., Guan, J.-L., Wang, Q.-P., Uehara, K., Yamada, S., Goto, N., Date, Y., Nakazato, M., Kojima, M., Kangawa, K., and Shioda, S. (2002). Immunocytochemical observation of ghrelin-containing neurons in the rat arcuate nucleus. Neuroscience Letters 321, 157-160.

(98) Seoane, L. M., López, M., Tovar, S., Casanueva, F. F., Señarís, R., and Diéguez, C. (2003). Agouti-Related Peptide, Neuropeptide Y, and Somatostatin-Producing Neurons Are Targets for Ghrelin Actions in the Rat Hypothalamus. Endocrinology 144, 544-551.

(99) Lage, R., Vázquez, M. J., Varela, L., Saha, A. K., Vidal-Puig, A., Nogueiras, R., Diéguez, C., and López, M. (2010). Ghrelin effects on neuropeptides in the rat hypothalamus depend on fatty acid metabolism actions on BSX but not on gender. The FASEB journal : official publication of the Federation of American Societies for Experimental Biology 24, 2670-2679.

(100) Chen, H. Y., Trumbauer, M. E., Chen, a. S., Weingarth, D. T., Adams, J. R., Frazier, E. G., Shen, Z., Marsh, D. J., Feighner, S. D., Guan, X. M., Ye, Z., Nargund, R. P., Smith, R. G., Van Der Ploeg, L. H. T., Howard, a. D., Macneil, D. J., and Qian, S. (2004). Orexigenic action of peripheral ghrelin is mediated by neuropeptide Y and agouti-related protein. Endocrinology 145, 2607-2612.

(101) Andrews, Z. B., Liu, Z.-W., Walllingford, N., Erion, D. M., Borok, E., Friedman, J. M., Tschöp, M. H., Shanabrough, M., Cline, G., Shulman, G. I., Coppola, A., Gao, X.-B., Horvath, T. L., and Diano, S. (2008). UCP2 mediates ghrelin's action on NPY/AgRP neurons by lowering free radicals. Nature 454, 846-851.

(102) Hewson, A. K., and Dickson, S. L. (2000). Systemic Administration of Ghrelin Induces Fos and Egr-1 Proteins in the Hypothalamic Arcuate Nucleus of Fasted and Fed Rats. Journal of Neuroendocrinology 12, 1047-1049. 
(103) Rüter, J., Kobelt, P., Tebbe, J. J., Avsar, Y., Veh, R., Wang, L., Klapp, B. F., Wiedenmann, B., Taché, Y., and Mönnikes, H. (2003). Intraperitoneal injection of ghrelin induces Fos expression in the paraventricular nucleus of the hypothalamus in rats. Brain Research 991, 26-33.

(104) Wang, L., Saint-Pierre, D. H., and Taché, Y. (2002). Peripheral ghrelin selectively increases Fos expression in neuropeptide $\mathrm{Y}$ - synthesizing neurons in mouse hypothalamic arcuate nucleus. Neuroscience Letters 325, 47-51.

(105) Date, Y., Murakami, N., Toshinai, K., Matsukura, S., Niijima, A., Matsuo, H., Kangawa, K., and Nakazato, M. (2002). The role of the gastric afferent vagal nerve in ghrelin-induced feeding and growth hormone secretion in rats. Gastroenterology 123, 1120-1128.

(106) Sakata, I., Yamazaki, M., Inoue, K., Hayashi, Y., Kangawa, K., and Sakai, T. (2003). Growth hormone secretagogue receptor expression in the cells of the stomach-projected afferent nerve in the rat nodose ganglion. Neuroscience Letters 342, 183-186.

(107) Zhang, W., Lin, T. R., Hu, Y., Fan, Y., Zhao, L., Stuenkel, E. L., and Mulholland, M. W. (2004). Ghrelin stimulates neurogenesis in the dorsal motor nucleus of the vagus. The Journal of Physiology 559, 729-737.

(108) Stapleton, D., Mitchelhill, K. I., Gao, G., Widmer, J., Michell, B. J., Teh, T., House, C. M., Fernandez, C. S., Cox, T., Witters, L. A., and Kemp, B. E. (1996). Mammalian AMP-activated Protein Kinase Subfamily. Journal of Biological Chemistry 271, 611-614.

(109) Quentin, T., Kitz, J., Steinmetz, M., Poppe, A., Bär, K., and Krätzner, R. (2011). Different expression of the catalytic alpha subunits of the AMP activated protein kinase-an immunohistochemical study in human tissue. Histology and histopathology 26, 589-596.

(110) Andersson, U., Filipsson, K., Abbott, C. R., Woods, A., Smith, K., Bloom, S. R., Carling, D., and Small, C. J. (2004). AMP-activated Protein Kinase Plays a Role in the Control of Food Intake. Journal of Biological Chemistry 279, 12005-12008.

(111) Minokoshi, Y., Alquier, T., Furukawa, N., Kim, Y.-B., Lee, A., Xue, B., Mu, J., Foufelle, F., Ferré, P., Birnbaum, M. J., Stuck, B. J., and Kahn, B. B. (2004). AMP-kinase regulates food intake by responding to hormonal and nutrient signals in the hypothalamus. Nature 428, 569-574.

(112) Hardie, D., Scott, J. W., Pan, D. A., and Hudson, E. R. (2003). Management of cellular energy by the AMP-activated protein kinase system. FEBS Letters $546,113-120$. 
(113) Ramamurthy, S., and Ronnett, G. (2012). AMP-Activated Protein Kinase (AMPK) and Energy-Sensing in the Brain. Experimental Neurobiology 21, 52-60.

(114) Hardie, D. G., Ross, F. A., and Hawley, S. A. (2012). AMPK: a nutrient and energy sensor that maintains energy homeostasis., English Nature Reviews Molecular Cell Biology 13, 251+.

(115) Kola, B., Hubina, E., Tucci, S. a., Kirkham, T. C., Garcia, E. a., Mitchell, S. E., Williams, L. M., Hawley, S. a., Hardie, D. G., Grossman, A. B., and Korbonits, M. (2005). Cannabinoids and ghrelin have both central and peripheral metabolic and cardiac effects via AMP-activated protein kinase. Journal of Biological Chemistry 280, 25196-25201.

(116) Wolfgang, M. J., and Lane, M. D. (2006). The Role of Hypothalamic Malonyl-CoA in Energy Homeostasis. Journal of Biological Chemistry 281, 37265-37269.

(117) Shimizu-Albergine, M., Ippolito, D. L., and Beavo, J. a. (2001). Downregulation of fasting-induced cAMP response element-mediated gene induction by leptin in neuropeptide $\mathrm{Y}$ neurons of the arcuate nucleus. The Journal of Neuroscience 21, 1238-1246.

(118) Kitamura, T., Feng, Y., Kitamura, Y. I., Chua, S. C., Xu, A. W., Barsh, G. S., Rossetti, L., and Accili, D. (2006). Forkhead protein FoxO1 mediates Agrpdependent effects of leptin on food intake. Nature medicine 12, 534-540.

(119) Nogueiras, R., Tovar, S., Mitchell, S. E., Rayner, D. V., Archer, Z. a., Dieguez, C., and Williams, L. M. (2004). Regulation of Growth Hormone Secretagogue Receptor Gene Expression in the Arcuate Nuclei of the Rat by Leptin and Ghrelin. Diabetes 53, 2552-2558.

(120) Sakkou, M., Wiedmer, P., Anlag, K., Hamm, A., Seuntjens, E., Ettwiller, L., Tschöp, M. H., and Treier, M. (2007). A Role for Brain-Specific Homeobox Factor Bsx in the Control of Hyperphagia and Locomotory Behavior. Cell Metabolism 5, 450-463.

(121) Nogueiras, R., López, M., Lage, R., Perez-Tilve, D., Pfluger, P., MendietaZerón, H., Sakkou, M., Wiedmer, P., Benoit, S. C., Datta, R., Dong, J. Z., Culler, M., Sleeman, M., Vidal-Puig, A., Horvath, T., Treier, M., Diéguez, C., and Tschöp, M. H. (2008). Bsx, a novel hypothalamic factor linking feeding with locomotor activity, is regulated by energy availability. Endocrinology 149, 3009-3015. 
(122) Ren, H., Orozco, I. J., Su, Y., Suyama, S., Gutiérrez-Juárez, R., Horvath, T. L., Wardlaw, S. L., Plum, L., Arancio, O., and Accili, D. (2012). FoxO1 target Gpr17 activates AgRP neurons to regulate food intake. Cell 149, 1314-1326.

(123) Accili, D., Aizawa, K. S., Cawley, N. X., DePinho, R. A., Dutia, R., Kim, A. J., Lin, H. V., Loh, Y. P., Matsumoto, M., Paik, J.-H., Plum, L., Tanaka, J., and Wardlaw, S. L. (2009). The obesity susceptibility gene Cpe links FoxO1 signaling in hypothalamic pro-opiomelanocortin neurons with regulation of food intake., English Nature Medicine 15, 1195+.

(124) Belgardt, B. F., Husch, A., Rother, E., Ernst, M. B., Wunderlich, F. T., Hampel, B., Klöckener, T., Alessi, D., Kloppenburg, P., and Brüning, J. C. (2008). PDK1 Deficiency in POMC-Expressing Cells Reveals FOXO1-Dependent and -Independent Pathways in Control of Energy Homeostasis and Stress Response. Cell Metabolism 7, 291-301.

(125) Ernst, M. B., Wunderlich, C. M., Hess, S., Paehler, M., Mesaros, A., Koralov, S. B., Kleinridders, A., Husch, A., Münzberg, H., Hampel, B., Alber, J., Kloppenburg, P., Brüning, J. C., and Wunderlich, F. T. (2009). Enhanced Stat3 activation in POMC neurons provokes negative feedback inhibition of leptin and insulin signaling in obesity. The Journal of neuroscience : the official journal of the Society for Neuroscience 29, 11582-11593.

(126) Ollmann, M. M., Wilson, B. D., and Barsh, G. S. (1997). Antagonism of central melanocortin receptors in vitro and in vivo by agouti-related protein., English Science 278, 135+.

(127) Kojima, M., and Kangawa, K., Ghrelin: Structure and Function; 2, 2005; Vol. 85, pp 495-522.

(128) Taylor, M. S., Hwang, Y., Hsiao, P.-Y., Boeke, J. D., and Cole, P. A. In Methods in Enzymology, Masayasu, K., and Kenji, K., Eds.; Academic Press: 2012; Vol. Volume 514, pp 205-228.

(129) Galas, L., Chartrel, N., Kojima, M., Kangawa, K., and Vaudry, H. (2002). Immunohistochemical localization and biochemical characterization of ghrelin in the brain and stomach of the frog Rana esculenta. The Journal of Comparative Neurology 450, 34-44.

(130) Kaiya, H., Kojima, M., Hosoda, H., Koda, A., Yamamoto, K., Kitajima, Y., Matsumoto, M., Minamitake, Y., Kikuyama, S., and Kangawa, K. (2001). Bullfrog Ghrelin Is Modified by n-Octanoic Acid at Its Third Threonine Residue. Journal of Biological Chemistry 276, 40441-40448. 
(131) Kaiya, H., van der Geyten, S., Kojima, M., Hosoda, H., Kitajima, Y., Matsumoto, M., Geelissen, S., Darras, V. M., and Kangawa, K. (2002). Chicken Ghrelin: Purification, cDNA Cloning, and Biological Activity. Endocrinology 143, 3454-3463.

(132) Kaiya, H., Kojima, M., Hosoda, H., Moriyama, S., Takahashi, A., Kawauchi, H., and Kangawa, K. (2003). Peptide Purification, Complementary Deoxyribonucleic Acid (DNA) and Genomic DNA Cloning, and Functional Characterization of Ghrelin in Rainbow Trout. Endocrinology 144, 5215-5226.

(133) Kaiya, H., Kojima, M., Hosoda, H., Riley, L. G., Hirano, T., Grau, E. G., and Kangawa, K. (2003). Identification of tilapia ghrelin and its effects on growth hormone and prolactin release in the tilapia, Oreochromis mossambicus. Comparative Biochemistry and Physiology Part B: Biochemistry and Molecular Biology 135, 421-429.

(134) Kaiya, H., Kojima, M., Hosoda, H., Riley, L. G., Hirano, T., Grau, E. G., and Kangawa, K. (2003). Amidated fish ghrelin: purification, cDNA cloning in the Japanese eel and its biological activity. Journal of Endocrinology 176, 415-423.

(135) Parhar, I. S., Sato, H., and Sakuma, Y. (2003). Ghrelin gene in cichlid fish is modulated by sex and development. Biochemical and Biophysical Research Communications 305, 169-175.

(136) Unniappan, S., Lin, X., Cervini, L., Rivier, J., Kaiya, H., Kangawa, K., and Peter, R. E. (2002). Goldfish Ghrelin: Molecular Characterization of the Complementary Deoxyribonucleic Acid, Partial Gene Structure and Evidence for Its Stimulatory Role in Food Intake. Endocrinology 143, 4143-4146.

(137) Gahete, M. D., Córdoba-Chacón, J., Salvatori, R., Castaño, J. P., Kineman, R. D., and Luque, R. M. (2010). Metabolic regulation of ghrelin O-acyl transferase (GOAT) expression in the mouse hypothalamus, pituitary, and stomach. Molecular and Cellular Endocrinology 317, 154-160.

(138) Stengel, A., Goebel, M., Wang, L., Taché, Y., Sachs, G., and Lambrecht, N. W. G. (2010). Differential distribution of ghrelin-O-acyltransferase (GOAT) immunoreactive cells in the mouse and rat gastric oxyntic mucosa. Biochemical and Biophysical Research Communications 392, 67-71.

(139) Rucinski, M., Ziolkowska, A., Szyszka, M., Hochol, A., and Malendowicz, L. K. (2012). Evidence suggesting that ghrelin O-acyl transferase inhibitor acts at the hypothalamus to inhibit hypothalamo-pituitary-adrenocortical axis function in the rat. Peptides 35, 149-159.

(140) Zhu, X., Cao, Y., Voodg, K., and Steiner, D. F. (2006). On the Processing of Proghrelin to Ghrelin. Journal of Biological Chemistry 281, 38867-38870. 
(141) Hofmann, K. (2000). A superfamily of membrane-bound O-acyltransferases with implications for Wnt signaling. Trends in Biochemical Sciences 25, 111112.

(142) Walia, P., Asadi, A., Kieffer, T. J., Johnson, J. D., and Chanoine, J.-P. (2009). Ontogeny of Ghrelin, Obestatin, Preproghrelin, and Prohormone Convertases in Rat Pancreas and Stomach. Pediatr Res 65, 39-44.

(143) Ugleholdt, R., Zhu, X., Deacon, C. F., Ørskov, C., Steiner, D. F., and Holst, J. J. (2004). Impaired Intestinal Proglucagon Processing in Mice Lacking Prohormone Convertase 1. Endocrinology 145, 1349-1355.

(144) Yoon, J., and Beinfeld, M. C. (1997). Prohormone Convertase 1 Is Necessary for the Formation of Cholecystokinin 8 in Rin5F and STC-1 Cells. Journal of Biological Chemistry 272, 9450-9456.

(145) Zhang, J. V., Ren, P.-G., Avsian-Kretchmer, O., Luo, C.-W., Rauch, R., Klein, C., and Aaron, J. W. H. (2005). Obestatin, a Peptide Encoded by the Ghrelin Gene, Opposes Ghrelin's Effects on Food Intake. Science 310, 996-999.

(146) Chartrel, N., Alvear-Perez, R., Leprince, J., Iturrioz, X., Goazigo, A. R.-L., Audinot, V., Chomarat, P., Coge, F., Nosjean, O., Rodriguez, M., Galizzi, J. P., Boutin, J. A., Vaudry, H., and Llorens-Cortes, C. (2007). Comment on "Obestatin, a Peptide Encoded by the Ghrelin Gene, Opposes Ghrelin's Effects on Food Intake". Science 315, 766.

(147) Gourcerol, G., Coskun, T., Craft, L. S., Mayer, J. P., Heiman, M. L., Wang, L., Million, M., St.-Pierre, D. H., and Taché, Y. (2007). Preproghrelin-derived Peptide, Obestatin, Fails to Influence Food Intake in Lean or Obese Rodents. Obesity 15, 2643-2652.

(148) Holst, B., Egerod, K. L., Schild, E., Vickers, S. P., Cheetham, S., Gerlach, L.-O., Storjohann, L., Stidsen, C. E., Jones, R., Beck-Sickinger, A. G., and Schwartz, T. W. (2007). GPR39 Signaling Is Stimulated by Zinc Ions But Not by Obestatin. Endocrinology 148, 13-20.

(149) Lauwers, E., Landuyt, B., Arckens, L., Schoofs, L., and Luyten, W. (2006). Obestatin does not activate orphan $\mathrm{G}$ protein-coupled receptor GPR39. Biochemical and Biophysical Research Communications 351, 21-25.

(150) Nogueiras, R., Pfluger, P., Tovar, S., Arnold, M., Mitchell, S., Morris, A., Perez-Tilve, D., Vázquez, M. J., Wiedmer, P., Castañeda, T. R., DiMarchi, R., Tschöp, M., Schurmann, A., Joost, H.-G., Williams, L. M., Langhans, W., and Diéguez, C. (2007). Effects of Obestatin on Energy Balance and Growth Hormone Secretion in Rodents. Endocrinology 148, 21-26. 
(151) Kineman, R. D., Gahete, M. D., and Luque, R. M. (2007). Identification of a mouse ghrelin gene transcript that contains intron 2 and is regulated in the pituitary and hypothalamus in response to metabolic stress. Journal of Molecular Endocrinology 38, 511-521.

(152) Seim, I., Collet, C., Herington, A., and Chopin, L. (2007). Revised genomic structure of the human ghrelin gene and identification of novel exons, alternative splice variants and natural antisense transcripts. BMC Genomics 8, 298.

(153) Nishi, Y., Hiejima, H., Hosoda, H., Kaiya, H., Mori, K., Fukue, Y., Yanase, T., Nawata, H., Kangawa, K., and Kojima, M. (2005). Ingested MediumChain Fatty Acids Are Directly Utilized for the Acyl Modification of Ghrelin. Endocrinology 146, 2255-2264.

(154) Taylor, M. S., Ruch, T. R., Hsiao, P.-Y., Hwang, Y., Zhang, P., Dai, L., Huang, C. R. L., Berndsen, C. E., Kim, M.-S., Pandey, A., Wolberger, C., Marmorstein, R., Machamer, C., Boeke, J. D., and Cole, P. A. (2013). Architectural Organization of the Metabolic Regulatory Enzyme Ghrelin-O-Acyltransferase. Journal of Biological Chemistry, DOI: 10.1074/jbc.M113.510313.

(155) Heppner, K. M., Chaudhary, N., Müller, T. D., Kirchner, H., Habegger, K. M., Ottaway, N., Smiley, D. L., DiMarchi, R., Hofmann, S. M., Woods, S. C., Sivertsen, B., Holst, B., Pfluger, P. T., Perez-Tilve, D., and Tschöp, M. H. (2012). Acylation Type Determines Ghrelin's Effects on Energy Homeostasis in Rodents. Endocrinology 153, 4687-4695.

(156) Grossauer, J., Kosol, S., Schrank, E., and Zangger, K. (2010). The peptide hormone ghrelin binds to membrane-mimetics via its octanoyl chain and an adjacent phenylalanine. Bioorganic \& Medicinal Chemistry 18, 5483-5488.

(157) Silva Elipe, M. V., Bednarek, M. A., and Gao, Y.-D. (2001). 1H NMR structural analysis of human ghrelin and its six truncated analogs. Biopolymers 59, 489-501.

(158) Bednarek, M. A., Feighner, S. D., Pong, S.-S., McKee, K. K., Hreniuk, D. L., Silva, M. V., Warren, V. A., Howard, A. D., Van der Ploeg, L. H. Y., and Heck, J. V. (2000). Structure-Function Studies on the New Growth Hormone-Releasing Peptide, Ghrelin: Minimal Sequence of Ghrelin Necessary for Activation of Growth Hormone Secretagogue Receptor 1a. Journal of Medicinal Chemistry 43, 4370-4376.

(159) Yang, J., Zhao, T.-J., Goldstein, J. L., and Brown, M. S. (2008). Inhibition of ghrelin O-acyltransferase (GOAT) by octanoylated pentapeptides. Proceedings of the National Academy of Sciences 105, 10750-10755. 
(160) Darling, J. E., Prybolsky, E. P., Sieburg, M., and Hougland, J. L. (2013). A fluorescent peptide substrate facilitates investigation of ghrelin recognition and acylation by ghrelin O-acyltransferase. Analytical Biochemistry 437, 68-76.

(161) Darling, J. E., Zhao, F., Loftus, R. J., Patton, L. M., Gibbs, R. a., and Hougland, J. L. (2015). Structure-Activity Analysis of Human Ghrelin O-Acyltransferase Reveals Chemical Determinants of Ghrelin Selectivity and Acyl Group Recognition. Biochemistry 54, 1100-1110.

(162) Delhanty, P. J. D., Huisman, M., Baldeon-Rojas, L. Y., van den Berge, I., Grefhorst, A., Abribat, T., Leenen, P. J. M., Themmen, A. P. N., and van der Lely, A.-J. (2013). Des-acyl ghrelin analogs prevent high-fat-diet-induced dysregulation of glucose homeostasis. The FASEB Journal 27, 1690-1700.

(163) Shindou, H., Eto, M., Morimoto, R., and Shimizu, T. (2009). Identification of membrane O-acyltransferase family motifs. Biochemical and Biophysical Research Communications 383, 320-325.

(164) Chang, C. C. Y., Sun, J., and Chang, T.-Y. (2011). Membrane-bound Oacyltransferases (MBOATs). Frontiers in Biology 6, 177-182.

(165) Pagac, M., de la Mora, H. V., Duperrex, C., Roubaty, C., Vionnet, C., and Conzelmann, A. (2011). Topology of 1-Acyl-sn-glycerol-3-phosphate Acyltransferases SLC1 and ALE1 and Related Membrane-bound OAcyltransferases (MBOATs) of Saccharomyces cerevisiae. Journal of Biological Chemistry 286, 36438-36447.

(166) Lin, S., Lu, X., Chang, C. C. Y., and Chang, T.-Y. (2003). Human AcylCoenzyme A:Cholesterol Acyltransferase Expressed in Chinese Hamster Ovary Cells: Membrane Topology and Active Site Location. Molecular Biology of the Cell 14, 2447-2460.

(167) Guo, Z.-Y., Lin, S., Heinen, J. A., Chang, C. C. Y., and Chang, T.-Y. (2005). The Active Site His-460 of Human Acyl-coenzyme A:Cholesterol Acyltransferase 1 Resides in a Hitherto Undisclosed Transmembrane Domain. Journal of Biological Chemistry 280, 37814-37826.

(168) Bosson, R., Jaquenoud, M., and Conzelmann, A. (2006). GUP1 of Saccharomyces cerevisiae Encodes an O-Acyltransferase Involved in Remodeling of the GPI Anchor. Molecular Biology of the Cell 17, 2636-2645.

(169) Lee, H.-C., Inoue, T., Imae, R., Kono, N., Shirae, S., Matsuda, S., GengyoAndo, K., Mitani, S., and Arai, H. (2008). Caenorhabditis elegans mboa-7, a Member of the MBOAT Family, Is Required for Selective Incorporation of Polyunsaturated Fatty Acids into Phosphatidylinositol. Molecular Biology of the Cell 19, 1174-1184. 
(170) McFie, P. J., Stone, S. L., Banman, S. L., and Stone, S. J. (2010). Topological Orientation of Acyl-CoA:Diacylglycerol Acyltransferase-1 (DGAT1) and Identification of a Putative Active Site Histidine and the Role of the N Terminus in Dimer/Tetramer Formation. Journal of Biological Chemistry 285, 37377-37387.

(171) Shen, H., and Chou, J. J. (2008). Membrain: Improving the accuracy of predicting transmembrane helices. PLoS ONE 3, DOI: 10.1371 / journal . pone.0002399.

(172) Nugent, T., and Jones, D. T. (2012). Detecting pore-lining regions in transmembrane protein sequences. BMC Bioinformatics 13, 169.

(173) Holst, B., Cygankiewicz, A., Jensen, T. H., Ankersen, M., and Schwartz, T. W. (2003). High constitutive signaling of the ghrelin receptor-identification of a potent inverse agonist. Molecular endocrinology (Baltimore, Md.) 17, 22012210.

(174) Holst, B., Holliday, N. D., Bach, A., Elling, C. E., Cox, H. M., and Schwartz, T. W. (2004). Common Structural Basis for Constitutive Activity of the Ghrelin Receptor Family. The Journal of biological chemistry 279, 53806-53817.

(175) Pantel, J., Legendre, M., Cabrol, S., Hilal, L., Hajaji, Y., Morisset, S., Nivot, S., Vie-Luton, M.-P., Grouselle, D., de Kerdanet, M., Kadiri, A., Epelbaum, J., Le Bouc, Y., and Amselem, S. (2006). Loss of constitutive activity of the growth hormone secretagogue receptor in familial short stature. The Journal of clinical investigation 116, 760-8.

(176) Krishna, A. G., Menon, S. T., Terry, T. J., and Sakmar, T. P. (2002). Evidence That Helix 8 of Rhodopsin Acts as a Membrane-Dependent Conformational Switch $\dagger$. Biochemistry 41, 8298-8309.

(177) Hubbell, W. L., Altenbach, C., Hubbell, C. M., and Khorana, H. In Membrane Proteins, Chemistry, B. T. .-.-. A. i. P., Ed.; Academic Press: 2003; Vol. Volume 63, pp 243-290.

(178) Fritze, O., Filipek, S., Kuksa, V., Palczewski, K., Hofmann, K. P., and Ernst, O. P. (2003). Role of the conserved $\operatorname{NPxxY}(\mathrm{x})(5,6) \mathrm{F}$ motif in the rhodopsin ground state and during activation. Proceedings of the National Academy of Sciences of the United States of America 100, 2290-2295.

(179) Elling, C. E., Frimurer, T. M., Gerlach, L.-O., Jorgensen, R., Holst, B., and Schwartz, T. W. (2006). Metal Ion Site Engineering Indicates a Global Toggle Switch Model for Seven-transmembrane Receptor Activation. Journal of Biological Chemistry 281, 17337-17346. 
(180) Dougherty, D. A. (2007). Cation- $\pi$ Interactions Involving Aromatic Amino Acids. The Journal of Nutrition 137, 1504S-1508S.

(181) Mellman, I. (1996). Endocytosis and molecular sorting. Annual Review of Cell and Developmental Biology 12, 575-625.

(182) Banks, W. a., Op, M. T., Robinson, S. M., and Heiman, M. L. (2002). Extent and Direction of Ghrelin Transport Across the Blood- Brain Barrier Is Determined by Its Unique Primary Structure. The Journal of pharmacology and experimental therapeutics 302, 822-827.

(183) Banks, W. a., Burney, B. O., and Robinson, S. M. (2008). Effects of triglycerides, obesity, and starvation on ghrelin transport across the blood-brain barrier. Peptides 29, 2061-2065.

(184) Banks, W. a., DiPalma, C. R., and Farrell, C. L. (1999). Impaired transport of leptin across the blood-brain barrier in obesity. Peptides 20, 1341-1345.

(185) Banks, W. A., and Farrell, C. L. (2003). Impaired transport of leptin across the blood-brain barrier in obesity is acquired and reversible. American Journal of Physiology - Endocrinology and Metabolism 285, E10-E15.

(186) Banks, W. A., Coon, A. B., Moinuddin, A., Morley, J. E., Nakaoke, R., Robinson, S. M., and Shultz, J. M. B. T. .-.-. D. (2004). Triglycerides induce leptin resistance at the blood-brain barrier., English Diabetes 53, $1253+$.

(187) Ouyang, S., Hsuchou, H., Kastin, A. J., Wang, Y., Yu, C., and Pan, W. (2013). Diet-induced obesity suppresses expression of many proteins at the bloodbrain barrier. Journal of cerebral blood flow and metabolism : official journal of the International Society of Cerebral Blood Flow and Metabolism 34, 1-9.

(188) Hahn, T. M., Breininger, J. F., Baskin, D. G., and Schwartz, M. W. (1998). Coexpression of Agrp and NPY in fasting-activated hypothalamic neurons. Nat Neurosci 1, 271-272.

(189) Baskin, D. G., Breininger, J. F., and Schwartz, M. W. (1999). Leptin receptor mRNA identifies a subpopulation of neuropeptide $\mathrm{Y}$ neurons activated by fasting in rat hypothalamus. Diabetes $48,828-833$.

(190) Schwartz, M. W., Seeley, R. J., Woods, S. C., Weigle, D. S., Campfield, L. A., Burn, P., and Baskin, D. G. (1997). Leptin Increases Hypothalamic Pro-opiomelanocortin mRNA Expression in the Rostral Arcuate Nucleus. Diabetes 46, 2119-2123.

(191) Mercer, J. G., Hoggard, N., Williams, L. M., Lawrence, C. B., Hannah, L. T., Morgan, P. J., and Trayhurn, P. (1996). Coexpression of Leptin Receptor and Preproneuropeptide Y mRNA in Arcuate Nucleus of Mouse Hypothalamus. Journal of Neuroendocrinology 8, 733-735. 
(192) Mizuno, T. M., Kleopoulos, S. P., Bergen, H. T., Roberts, J. L., Priest, C. A., and Mobbs, C. V. (1998). Hypothalamic Pro-Opiomelanocortin mRNA Is Reduced By Fasting in ob/ob and db/db Mice, but Is Stimulated by Leptin. Diabetes 47, 294-297.

(193) Erickson, J. C., Hollopeter, G., and Palmiter, R. D. (1996). Attenuation of the Obesity Syndrome of ob/ob Mice by the Loss of Neuropeptide Y. Science 274, 1704-1707.

(194) Rosenbaum, M., Murphy, E. M., Heymsfield, S. B., Matthews, D. E., and Leibel, R. L. (2002). Low Dose Leptin Administration Reverses Effects of Sustained Weight-Reduction on Energy Expenditure and Circulating Concentrations of Thyroid Hormones. The Journal of Clinical Endocrinology \& Metabolism 87, 2391.

(195) Rosenbaum, M., Sy, M., Pavlovich, K., Leibel, R. L., and Hirsch, J. (2008). Leptin reverses weight loss-induced changes in regional neural activity responses to visual food stimuli. The Journal of Clinical Investigation 118, 25832591.

(196) Leibel, R. L., Rosenbaum, M., and Hirsch, J. (1995). Changes in energy expenditure resulting from altered body weight. The New England journal of medicine 332, 621-628.

(197) Rosenbaum, M., Vandenborne, K., Goldsmith, R., Simoneau, J.-A., Heymsfield, S., Joanisse, D. R., Hirsch, J., Murphy, E., Matthews, D., Segal, K. R., and Leibel, R. L. (2003). Effects of experimental weight perturbation on skeletal muscle work efficiency in human subjects. American Journal of Physiology Regulatory, Integrative and Comparative Physiology 285, R183-R192.

(198) Doucet, E., Imbeault, P., St-Pierre, S., Alméras, N., Mauriège, P., Després, J.-P., Bouchard, C., and Tremblay, A. (2003). Greater than predicted decrease in energy expenditure during exercise after body weight loss in obese men. Clinical science (London, England: 1979) 105, 89-95.

(199) Arone, L. J., Mackintosh, R., Rosenbaum, M., Leibel, R. L., and Hirsch, J. (1995). Autonomic nervous system activity in weight gain and weight loss. American Journal of Physiology - Regulatory, Integrative and Comparative Physiology 269, R222-R225.

(200) Rosenbaum, M., Hirsch, J., Murphy, E., and Leibel, R. L. (2000). Effects of changes in body weight on carbohydrate metabolism, catecholamine excretion, and thyroid function. The American Journal of Clinical Nutrition 71, 14211432 . 
(201) Rissanen, P., Franssila-Kallunki, a., and Rissanen, a. (2001). Cardiac parasympathetic activity is increased by weight loss in healthy obese women. Obesity research 9, 637-643.

(202) Emdin, M., Gastaldelli, A., Muscelli, E., Macerata, A., Natali, A., Camastra, S., and Ferrannini, E. (2001). Hyperinsulinemia and Autonomic Nervous System Dysfunction in Obesity: Effects of Weight Loss. Circulation 103, 513519.

(203) Rosenbaum, M., Goldsmith, R., Bloomfield, D., Magnano, A., Weimer, L., Heymsfield, S., Gallagher, D., Mayer, L., Murphy, E., and Leibel, R. L. (2005). Low-dose leptin reverses skeletal muscle, autonomic, and neuroendocrine adaptations to maintenance of reduced weight. Journal of Clinical Investigation 115, 3579-3586.

(204) Frederich, R. C., Hamann, A., Anderson, S., Lollmann, B., Lowell, B. B., and Flier, J. S. (1995). Leptin levels reflect body lipid content in mice: Evidence for diet-induced resistance to leptin action. Nat Med 1, 1311-1314.

(205) Maffei, M., Halaas, J., Ravussin, E., Pratley, R. E., Lee, G. H., Zhang, Y., Fei, H., Kim, S., Lallone, R., Ranganathan, S., Kern, P. A., and Friedman, J. M. (1995). Leptin levels in human and rodent: Measurement of plasma leptin and ob RNA in obese and weight-reduced subjects. Nat Med 1, 1155-1161.

(206) Halaas, J. L., Boozer, C., Blair-West, J., Fidahusein, N., Denton, D. A., and Friedman, J. M. (1997). Physiological response to long-term peripheral and central leptin infusion in lean and obese mice. Proceedings of the National Academy of Sciences of the United States of America 94, 8878-8883.

(207) Schwartz, M. W., Peskind, E., Raskind, M., Boyko, E. J., and Porte, D. (1996). Cerebrospinal fluid leptin levels: Relationship to plasma levels and to adiposity in humans. Nat Med 2, 589-593.

(208) Considine, R. V., Caro, J. F., Considine, E. L., Williams, C. J., and Hyde, T. M. (1996). Identification of Incidental Sequence Polymorphisms and Absence of the $\mathrm{db} / \mathrm{db}$ Mouse and fa/fa Rat Mutations. Diabetes 45, 992-994.

(209) Heymsfield, S., Greenberg, A., Fujioka, K., Dixon, R., Kushner, R., Hunt, T., Lubina, J., Patane, J., Self, B., Hunt, P., and McCamish, M. (1999). Recombinant leptin for weight loss in obese and lean adults: A randomized, controlled, dose-escalation trial. JAMA 282, 1568-1575.

(210) Hukshorn, C. J., Saris, W. H. M., Westerterp-Plantenga, M. S., Farid, A. R., Smith, F. J., and Campfield, L. A. (2000). Weekly Subcutaneous Pegylated Recombinant Native Human Leptin (PEG-OB) Administration in Obese Men. The Journal of Clinical Endocrinology \& Metabolism 85, 4003-4009. 
(211) Westerterp-Plantenga, M. S., Saris, W. H. M., Hukshorn, C. J., and Campfield, L. A. (2001). Effects of weekly administration of pegylated recombinant human $\mathrm{OB}$ protein on appetite profile and energy metabolism in obese men. The American Journal of Clinical Nutrition 74, 426-434.

(212) Myers, M. G., Cowley, M. A., and Münzberg, H. (2008). Mechanisms of Leptin Action and Leptin Resistance. Annual Review of Physiology 70, 537-556.

(213) Münzberg, H. (2008). Differential leptin access into the brain - A hierarchical organization of hypothalamic leptin target sites? Physiology \& Behavior 94, 664-669.

(214) Beck, B., Musse, N., and Stricker-Krongrad, A. (2002). Ghrelin, Macronutrient Intake and Dietary Preferences in Long-Evans Rats. Biochemical and Biophysical Research Communications 292, 1031-1035.

(215) Morton, G., and Schwartz, M. (2006). Central nervous system control of food intake and body weight. Nature Reviews 443, 289-95.

(216) Bouret, S. G., Draper, S. J., and Simerly, R. B. (2004). Trophic action of leptin on hypothalamic neurons that regulate feeding. Science (New York, N.Y.) 304, 108-110.

(217) Bouret, S. G., Gorski, J. N., Patterson, C. M., Chen, S., Levin, B. E., and Simerly, R. B. (2008). Hypothalamic Neural Projections Are Permanently Disrupted in Diet-Induced Obese Rats. Cell Metabolism 7, 179-185.

(218) Pinto, S., Roseberry, A. G., and Liu, H. (2004). Rapid Rewiring of Arcuate Nucleus Feeding Circuits by Leptin. Science 304, 110-115.

(219) Ahima, R. S., Prabakaran, D., and Flier, J. S. (1998). Postnatal leptin surge and regulation of circadian rhythm of leptin by feeding. Implications for energy homeostasis and neuroendocrine function. The Journal of Clinical Investigation $101,1020-1027$.

(220) Chen, L., Xing, T., Wang, M., Miao, Y., Tang, M., Chen, J., Li, G., and Ruan, D.-Y. (2011). Local infusion of ghrelin enhanced hippocampal synaptic plasticity and spatial memory through activation of phosphoinositide 3-kinase in the dentate gyrus of adult rats. European Journal of Neuroscience 33, 266-275.

(221) Ariyasu, H., Takaya, K., Hosoda, H., Iwakura, H., Ebihara, K., Mori, K., Ogawa, Y., Hosoda, K., Akamizu, T., Kojima, M., Kangawa, K., and Nakao, K. (2002). Delayed Short-Term Secretory Regulation of Ghrelin in Obese Animals: Evidenced by a Specific RIA for the Active Form of Ghrelin. Endocrinology 143, 3341-3350. 
(222) Rigamonti, a. E., Pincelli, a. I., Corrá, B., Viarengo, R., Bonomo, S. M., Galimberti, D., Scacchi, M., Scarpini, E., Cavagnini, F., and Müller, E. E. (2002). Plasma ghrelin concentrations in elderly subjects: Comparison with anorexic and obese patients. Journal of Endocrinology 175, 1-5.

(223) Perreault, M., Istrate, N., Wang, L., Nichols, a. J., Tozzo, E., and StrickerKrongrad, a. (2004). Resistance to the orexigenic effect of ghrelin in dietaryinduced obesity in mice: reversal upon weight loss. International Journal of Obesity 28, 879-885.

(224) Briggs, D. I., Enriori, P. J., Lemus, M. B., Cowley, M. A., and Andrews, Z. B. (2010). Diet-Induced Obesity Causes Ghrelin Resistance in Arcuate NPY/AgRP Neurons. Endocrinology 151, 4745-4755.

(225) Sturm, K., MacIntosh, C. G., Parker, B. A., Wishart, J., Horowitz, M., and Chapman, I. M. (2003). Appetite, Food Intake, and Plasma Concentrations of Cholecystokinin, Ghrelin, and Other Gastrointestinal Hormones in Undernourished Older Women and Well-Nourished Young and Older Women. The Journal of Clinical Endocrinology \& Metabolism 88, 3747-3755.

(226) Namkoong, C., Kim, M. S., Jang, P. G., Han, S. M., Park, H. S., Koh, E. H., Lee, W. J., Kim, J. Y., Park, I. S., Park, J. Y., and Lee, K. U. (2005). Enhanced Hypothalamic AMP-Activated Protein Kinase Activity Contributes to Hyperphagia in Diabetic Rats. Diabetes 54, 63-68.

(227) Martin, T. L. (2006). Diet-induced Obesity Alters AMP Kinase Activity in Hypothalamus and Skeletal Muscle. Journal of Biological Chemistry 281, 18933-18941.

(228) De Smet, B., Depoortere, I., Moechars, D., Swennen, Q., Moreaux, B., Cryns, K., Tack, J., Buyse, J., Coulie, B., and Peeters, T. L. (2006). Energy homeostasis and gastric emptying in ghrelin knockout mice. The Journal of pharmacology and experimental therapeutics 316, 431-439.

(229) Wortley, K. E., Anderson, K. D., Garcia, K., Murray, J. D., Malinova, L., Liu, R., Moncrieffe, M., Thabet, K., Cox, H. J., Yancopoulos, G. D., Wiegand, S. J., and Sleeman, M. W. (2004). Genetic deletion of ghrelin does not decrease food intake but influences metabolic fuel preference. Proceedings of the National Academy of Sciences of the United States of America 101, 8227-8232.

(230) Sun, Y., Ahmed, S., and Smith, R. G. (2003). Deletion of Ghrelin Impairs neither Growth nor Appetite. Molecular and Cellular Biology 23, 7973-7981.

(231) Wortley, K. E., del Rincon, J.-P., Murray, J. D., Garcia, K., Iida, K., Thorner, M. O., and Sleeman, M. W. (2005). Absence of ghrelin protects against earlyonset obesity. The Journal of Clinical Investigation 115, 3573-3578. 
(232) Sun, Y., Butte, N. F., Garcia, J. M., and Smith, R. G. (2008). Characterization of Adult Ghrelin and Ghrelin Receptor Knockout Mice under Positive and Negative Energy Balance. Endocrinology 149, 843-850.

(233) Kirchner, H., Gutierrez, J. a., Solenberg, P. J., Pfluger, P. T., Czyzyk, T. a., Willency, J. a., Schürmann, A., Joost, H.-G., Jandacek, R. J., Hale, J. E., Heiman, M. L., and Tschöp, M. H. (2009). GOAT links dietary lipids with the endocrine control of energy balance. Nature medicine 15, 741-745.

(234) Zhao, T.-J., Liang, G., Li, R. L., Xie, X., Sleeman, M. W., Murphy, A. J., Valenzuela, D. M., Yancopoulos, G. D., Goldstein, J. L., and Brown, M. S. (2010). Ghrelin O-acyltransferase (GOAT) is essential for growth hormonemediated survival of calorie-restricted mice. Proceedings of the National Academy of Sciences 107, 7467-7472.

(235) Garner, A. L., and Janda, K. D. (2010). cat-ELCCA: A Robust Method To Monitor the Fatty Acid Acyltransferase Activity of Ghrelin O-Acyltransferase (GOAT). Angewandte Chemie International Edition 49, 9630-9634.

(236) Barnett, B. P., Hwang, Y., Taylor, M. S., Kirchner, H., Pfluger, P. T., Bernard, V., Lin, Y.-y., Bowers, E. M., Mukherjee, C., Song, W.-J., Longo, P. A., Leahy, D. J., Hussain, M. A., Tschöp, M. H., Boeke, J. D., and Cole, P. A. (2010). Glucose and Weight Control in Mice with a Designed Ghrelin O-Acyltransferase Inhibitor. Science 330, 1689-1692.

(237) Zhao, F., Darling, J. E., Gibbs, R. a., and Hougland, J. L. (2015). A new class of ghrelin $\mathrm{O}$-acyltransferase inhibitors incorporating triazole-linked lipid mimetic groups. Bioorganic \& Medicinal Chemistry Letters 25, 2800-2803.

(238) Vieira, P., and Rajewsky, K. (1988). The half-lives of serum immunoglobulins in adult mice. European Journal of Immunology 18, 313-316.

(239) Spiegelberg, H. L., and Fishkin, B. G. (1972). The catabolism of human $\gamma \mathrm{G}$ immunoglobulins of different heavy chain subclasses. III. The catabolism of heavy chain disease proteins and of Fc fragments of myeloma proteins. Clinical and Experimental Immunology 10, 599-607.

(240) Mayorov, A. V., Amara, N., Chang, J. Y., Moss, J. a., Hixon, M. S., Ruiz, D. I., Meijler, M. M., Zorrilla, E. P., and Janda, K. D. (2008). Catalytic antibody degradation of ghrelin increases whole-body metabolic rate and reduces refeeding in fasting mice. Proceedings of the National Academy of Sciences of the United States of America 105, 17487-17492. 
(241) Lu, S.-C., Xu, J., Chinookoswong, N., Liu, S., Steavenson, S., Gegg, C., Brankow, D., Lindberg, R., Véniant, M., and Gu, W. (2009). An acylghrelin-specific neutralizing antibody inhibits the acute ghrelin-mediated orexigenic effects in mice. Molecular pharmacology 75, 901-907.

(242) Zakhari, J. S., Zorrilla, E. P., Zhou, B., Mayorov, A. V., and Janda, K. D. (2012). Oligoclonal antibody targeting ghrelin increases energy expenditure and reduces food intake in fasted mice. Molecular Pharmaceutics 9, 281-289.

(243) Bagnasco, M., Tulipano, G., Melis, M. R., Argiolas, A., Cocchi, D., and Muller, E. E. (2003). Endogenous ghrelin is an orexigenic peptide acting in the arcuate nucleus in response to fasting. Regulatory Peptides 111, 161-167.

(244) Schultz, P. G., and Lerner, R. A. (2002). Antibody catalysis: Completing the circle. Nature 418, 485.

(245) Vizcarra, J. a., Kirby, J. D., Kim, S. K., and Galyean, M. L. (2007). Active immunization against ghrelin decreases weight gain and alters plasma concentrations of growth hormone in growing pigs. Domestic Animal Endocrinology 33, 176-189.

(246) Zorrilla, E. P., Iwasaki, S., Moss, J. a., Chang, J., Otsuji, J., Inoue, K., Meijler, M. M., and Janda, K. D. (2006). Vaccination against weight gain. Proceedings of the National Academy of Sciences of the United States of America 103, 1322613231.

(247) Andrade, S., Pinho, F., Ribeiro, a. M., Carreira, M., Casanueva, F. F., Roy, P., and Monteiro, M. P. (2013). Immunization against active ghrelin using viruslike particles for obesity treatment. Curr Pharm Des 19, 6551-6558.

(248) Helmling, S., Maasch, C., Eulberg, D., Buchner, K., Schröder, W., Lange, C., Vonhoff, S., Wlotzka, B., Tschöp, M. H., Rosewicz, S., and Klussmann, S. (2004). Inhibition of ghrelin action in vitro and in vivo by an RNASpiegelmer. Proceedings of the National Academy of Sciences of the United States of America 101, 13174-13179.

(249) Shearman, L. P., Wang, S. P., Helmling, S., Stribling, D. S., Mazur, P., Ge, L., Wang, L., Klussmann, S., Macintyre, D. E., Howard, A. D., and Strack, A. M. (2006). Ghrelin neutralization by a ribonucleic acid-SPM ameliorates obesity in diet-induced obese mice. Endocrinology 147, 1517-1526.

(250) Jarosch, F., Buchner, K., and Klussmann, S. (2006). In vitro selection using a dual RNA library that allows primerless selection. Nucleic Acids Research 34, DOI: $10.1093 / \mathrm{nar} / \mathrm{gkl} 463$. 
(251) Kobelt, P., Helmling, S., Stengel, a., Wlotzka, B., Andresen, V., Klapp, B. F., Wiedenmann, B., Klussmann, S., and Mönnikes, H. (2006). Anti-ghrelin Spiegelmer NOX-B11 inhibits neurostimulatory and orexigenic effects of peripheral ghrelin in rats. Gut 55, 788-792.

(252) Sangiao-Alvarellos, S., Helmling, S., Vázquez, M. J., Klussmann, S., and Cordido, F. (2011). Ghrelin neutralization during fasting-refeeding cycle impairs the recuperation of body weight and alters hepatic energy metabolism. Molecular and Cellular Endocrinology 335, 177-188.

(253) Teubner, B. J. W., and Bartness, T. J. (2013). Anti-ghrelin Spiegelmer inhibits exogenous ghrelin-induced increases in food intake, hoarding, and neural activation, but not food deprivation-induced increases. American journal of physiology. Regulatory, integrative and comparative physiology 305, R323-33.

(254) Becskei, C., Bilik, K. U., Klussmann, S., Jarosch, F., Lutz, T. a., and Riediger, T. (2008). The anti-ghrelin spiegelmer NOX-B11-3 blocks ghrelin- but not fasting-induced neuronal activation in the hypothalamic arcuate nucleus. Journal of Neuroendocrinology 20, 85-92.

(255) Patterson, Z. Ghrelin - The Defender of Fat in the Face of Stress Implications in Obesity Treatment., Ph.D. Thesis, Carleton University, 2013.

(256) Julien, M., Kay, R. G., Delhanty, P. J. D., Allas, S., Granata, R., Barton, C., Constable, S., Ghigo, E., van der Lely, A. J., and Abribat, T. (2012). In vitro and in vivo stability and pharmacokinetic profile of unacylated ghrelin (UAG) analogues. European Journal of Pharmaceutical Sciences 47, 625-635.

(257) Mizutani, M., Atsuchi, K., Asakawa, A., Matsuda, N., Fujimura, M., Inui, A., Kato, I., and Fujimiya, M. (2009). Localization of acyl ghrelin- and des-acyl ghrelin-immunoreactive cells in the rat stomach and their responses to intragastric $\mathrm{pH}$. American Journal of Physiology: Gastrointestinal and Liver Physiology 297, G974-G980.

(258) Blum, I. D., Patterson, Z., Khazall, R., Lamont, E. W., Sleeman, M. W., Horvath, T. L., and Abizaid, A. (2009). Reduced anticipatory locomotor responses to scheduled meals in ghrelin receptor deficient mice. Neuroscience 164, 3519 .

(259) Drazen, D. L., Vahl, T. P., D’Alessio, D. A., Seeley, R. J., and Woods, S. C. (2006). Effects of a Fixed Meal Pattern on Ghrelin Secretion: Evidence for a Learned Response Independent of Nutrient Status. Endocrinology 147, 23-30.

(260) LeSauter, J., Hoque, N., Weintraub, M., Pfaff, D. W., and Silver, R. (2009). Stomach ghrelin-secreting cells as food-entrainable circadian clocks. Proceedings of the National Academy of Sciences 106, 13582-13587. 
(261) Chuang, J.-C., Sakata, I., Kohno, D., Perello, M., Osborne-Lawrence, S., Repa, J. J., and Zigman, J. M. (2011). Ghrelin Directly Stimulates Glucagon Secretion from Pancreatic $\alpha$-Cells. Molecular endocrinology (Baltimore, Md.) 25, 1600-1611.

(262) Gualillo, O., Lago, F., and Dieguez, C. (2008). Introducing GOAT: a target for obesity and anti-diabetic drugs? Trends in Pharmacological Sciences 29, 398401.

(263) Sun, Y., Asnicar, M., and Smith, R. G. (2007). Central and Peripheral Roles of Ghrelin on Glucose Homeostasis. Neuroendocrinology 86, 215-228.

(264) Yada, T., Dezaki, K., Sone, H., Koizumi, M., Damdindorj, B., Kakei, M. N., and Masafumi Ghrelin Regulates Insulin Release and Glycemia: Physiological Role and Therapeutic Potential., 2008.

(265) Dezaki, K., Kakei, M., and Yada, T. (2007). Ghrelin Uses Goi2 and Activates Voltage-Dependent K+ Channels to Attenuate Glucose-Induced Ca2+ Signaling and Insulin Release in Islet $\beta$-Cells: Novel Signal Transduction of Ghrelin. Diabetes 56, 2319-2327.

(266) McLaughlin, T., Abbasi, F., Lamendola, C., Frayo, R. S., and Cummings, D. E. (2004). Plasma Ghrelin Concentrations Are Decreased in InsulinResistant Obese Adults Relative to Equally Obese Insulin-Sensitive Controls. The Journal of Clinical Endocrinology \& Metabolism 89, 1630-1635.

(267) Walker, A. K., Rivera, P. D., Wang, Q., Chuang, J.-C., Tran, S., OsborneLawrence, S., Estill, S. J., Starwalt, R., Huntington, P., Morlock, L., Naidoo, J., Williams, N. S., Ready, J. M., Eisch, A. J., Pieper, A. A., and Zigman, J. M. (2015). The P7C3 class of neuroprotective compounds exerts antidepressant efficacy in mice by increasing hippocampal neurogenesis. Mol Psychiatry 20, 500-508.

(268) Patterson, Z. R., Ducharme, R., Anisman, H., and Abizaid, A. (2010). Altered metabolic and neurochemical responses to chronic unpredictable stressors in ghrelin receptor-deficient mice. European Journal of Neuroscience 32, 632-639.

(269) Patterson, Z. R., Khazall, R., MacKay, H., Anisman, H., and Abizaid, A. (2013). Central Ghrelin Signaling Mediates the Metabolic Response of C57BL/6 Male Mice to Chronic Social Defeat Stress. Endocrinology 154, 1080-1091.

(270) Spencer, S. J., Xu, L., Clarke, M. A., Lemus, M., Reichenbach, A., Geenen, B., Kozicz, T., and Andrews, Z. B. (2012). Ghrelin Regulates the HypothalamicPituitary-Adrenal Axis and Restricts Anxiety After Acute Stress. Biological Psychiatry 72, 457-465. 
(271) Currie, P. J., Khelemsky, R., Rigsbee, E. M., Dono, L. M., Coiro, C. D., Chapman, C. D., and Hinchcliff, K. (2012). Ghrelin is an orexigenic peptide and elicits anxiety-like behaviors following administration into discrete regions of the hypothalamus. Behavioural Brain Research 226, 96-105.

(272) King, S. J., Isaacs, A. M., O’Farrell, E., and Abizaid, A. (2011). Motivation to obtain preferred foods is enhanced by ghrelin in the ventral tegmental area. Hormones and Behavior 60, 572-580.

(273) Skibicka, K. P., Hansson, C., Alvarez-Crespo, M., Friberg, P. A., and Dickson, S. L. (2011). Ghrelin directly targets the ventral tegmental area to increase food motivation. Neuroscience 180, 129-137.

(274) Cowley, M. A., Smart, J. L., Rubinstein, M., Cerdan, M. G., Diano, S., Horvath, T. L., Cone, R. D., and Low, M. J. (2001). Leptin activates anorexigenic POMC neurons through a neural network in the arcuate nucleus. Nature 411, 480-484.

(275) Currie, P. J., Mirza, A., Fuld, R., Park, D., and Vasselli, J. R. (2005). Ghrelin is an orexigenic and metabolic signaling peptide in the arcuate and paraventricular nuclei. American Journal of Physiology - Regulatory, Integrative and Comparative Physiology 289, R353-R358.

(276) Date, Y., Shimbara, T., Koda, S., Toshinai, K., Ida, T., Murakami, N., Miyazato, M., Kokame, K., Ishizuka, Y., Ishida, Y., Kageyama, H., Shioda, S., Kangawa, K., and Nakazato, M. (2006). Peripheral ghrelin transmits orexigenic signals through the noradrenergic pathway from the hindbrain to the hypothalamus. Cell metabolism 4, 323-331.

(277) Healy, J. E., Bateman, J. L., Ostrom, C. E., and Florant, G. L. (2011). Peripheral ghrelin stimulates feeding behavior and positive energy balance in a sciurid hibernator. Hormones and Behavior 59, 512-519.

(278) Keen-Rhinehart, E., and Bartness, T. J. (2005). Peripheral ghrelin injections stimulate food intake, foraging, and food hoarding in Siberian hamsters. American Journal of Physiology - Regulatory, Integrative and Comparative Physiology 288, R716-R722.

(279) Tang-Christensen, M., Vrang, N., Ortmann, S., Bidlingmaier, M., Horvath, T. L., and Tschöp, M. (2004). Central Administration of Ghrelin and AgoutiRelated Protein (83-132) Increases Food Intake and Decreases Spontaneous Locomotor Activity in Rats. Endocrinology 145, 4645-4652. 
(280) Choi, K., Roh, S.-G., Hong, Y.-H., Shrestha, Y. B., Hishikawa, D., Chen, C., Kojima, M., Kangawa, K., and Sasaki, S.-I. (2003). The Role of Ghrelin and Growth Hormone Secretagogues Receptor on Rat Adipogenesis. Endocrinology 144, 754-759.

(281) Merkestein, M., Brans, M. A. D., Luijendijk, M. C. M., de Jong, J. W., Egecioglu, E., Dickson, S. L., and Adan, R. A. H. (2012). Ghrelin Mediates Anticipation to a Palatable Meal in Rats. Obesity 20, 963-971.

(282) Howard, A. D., Feighner, S. D., Cully, D. F., Arena, J. P., Liberator, P. A., Rosenblum, C. I., Hamelin, M., Hreniuk, D. L., Palyha, O. C., Anderson, J., Paress, P. S., Diaz, C., Chou, M., Liu, K. K., McKee, K. K., Pong, S.-S., Chaung, L.-Y., Elbrecht, A., Dashkevicz, M., Heavens, R., Rigby, M., Sirinathsinghji, D. J. S., Dean, D. C., Melillo, D. G., Patchett, A. A., Nargund, R., Griffin, P. R., DeMartino, J. A., Gupta, S. K., Schaeffer, J. M., Smith, R. G., and Van der Ploeg, L. H. T. (1996). A Receptor in Pituitary and Hypothalamus That Functions in Growth Hormone Release. Science 273, 974977.

(283) Zigman, J. M., Jones, J. E., Lee, C. E., Saper, C. B., and Elmquist, J. K. (2006). Expression of ghrelin receptor mRNA in the rat and the mouse brain. The Journal of Comparative Neurology 494, 528-548.

(284) Abizaid, A., and Horvath, T. L. (2008). Brain circuits regulating energy homeostasis. Regulatory Peptides 149, 3-10.

(285) Lim, C. T., Kola, B., Grossman, A., and Korbonits, M. (2011). The expression of ghrelin O-acyltransferase (GOAT) in human tissues. Endocrine Journal 58, $707-710$.

(286) Garner, A. L., and Janda, K. D. (2011). A small molecule antagonist of ghrelin O-acyltransferase (GOAT). Chem. Commun. 47, 7512-7514.

(287) Mani, B. K., Chuang, J.-C., Kjalarsdottir, L., Sakata, I., Walker, A. K., Kuperman, A., Osborne-Lawrence, S., Repa, J. J., and Zigman, J. M. (2014). Role of Calcium and EPAC in Norepinephrine-Induced Ghrelin Secretion. Endocrinology 155, 98-107.

(288) Zhao, T.-J., Sakata, I., Li, R. L., Liang, G., Richardson, J. A., Brown, M. S., Goldstein, J. L., and Zigman, J. M. (2010). Ghrelin secretion stimulated by $\beta 1$-adrenergic receptors in cultured ghrelinoma cells and in fasted mice. Proceedings of the National Academy of Sciences 107, 15868-15873.

(289) Pérez-Tilve, D., González-Matías, L., Alvarez-Crespo, M., Leiras, R., Tovar, S., Diéguez, C., and Mallo, F. (2007). Exendin-4 Potently Decreases Ghrelin Levels in Fasting Rats. Diabetes 56, 143-151. 
(290) Moulin, A., Ryan, J., Martinez, J., and Fehrentz, J.-A. (2007). Recent Developments in Ghrelin Receptor Ligands. ChemMedChem 2, 1242-1259.

(291) Jones, S. W., Christison, R., Bundell, K., Voyce, C. J., Brockbank, S. M. V., Newham, P., and Lindsay, M. A. (2005). Characterisation of cell-penetrating peptide-mediated peptide delivery. British Journal of Pharmacology 145, 10931102.

(292) Delhanty, P. J. D., Neggers, S. J., and van der Lely, A. J. (2012). MECHANISMS IN ENDOCRINOLOGY: Ghrelin: the differences between acyl- and des-acyl ghrelin. European Journal of Endocrinology 167, 601-608.

(293) Abizaid, A., and Anisman, H. (2014). Gut feelings about depression. Journal of Psychiatry \& Neuroscience: JPN 39, 364-366.

(294) Spencer, S. J., Emmerzaal, T. L., Kozicz, T., and Andrews, Z. B. (2015). Ghrelin's Role in the Hypothalamic-Pituitary-Adrenal Axis Stress Response: Implications for Mood Disorders. Biological Psychiatry 78, 19-27.

(295) Andrews, Z. B., Erion, D., Beiler, R., Liu, Z.-W., Abizaid, A., Zigman, J., Elsworth, J. D., Savitt, J. M., DiMarchi, R., Tschöp, M., Roth, R. H., Gao, X.-B., and Horvath, T. L. (2009). Ghrelin Promotes and Protects Nigrostriatal Dopamine Function via a UCP2-Dependent Mitochondrial Mechanism. The Journal of Neuroscience 29, 14057-14065.

(296) Peino, R., Baldelli, R., Rodriguez-Garcia, J., Rodriguez-Segade, S., Kojima, M., Kangawa, K., Arvat, E., Ghigo, E., Dieguez, C., and Casanueva, F. F. (2000). Ghrelin-induced growth hormone secretion in humans. European Journal of Endocrinology 143, R11-R14.

(297) Furness, J. B., Hunne, B., Matsuda, N., Yin, L., Russo, D., Kato, I., Fujimiya, M., Patterson, M., McLeod, J., Andrews, Z. B., and Bron, R. (2011). Investigation of the presence of ghrelin in the central nervous system of the rat and mouse. Neuroscience 193, 1-9.

(298) Gualillo, O., Caminos, J. E., Nogueiras, R., Seoane, L. M., Arvat, E., Ghigo, E., Casanueva, F. F., and Diéguez, C. (2002). Effect of food restriction on ghrelin in normal-cycling female rats and in pregnancy. Obesity Research 10, 682-687.

(299) Luque, R. M., Gahete, M. D., Hochgeschwender, U., and Kineman, R. D. (2006). Evidence that endogenous SST inhibits ACTH and ghrelin expression by independent pathways. American Journal of Physiology: Endocrinology and Metabolism 291, E395-E403. 
(300) Sakata, I., Nakano, Y., Osborne-Lawrence, S., Rovinsky, S. A., Lee, C. E., Perello, M., Anderson, J. G., Coppari, R., Xiao, G., Lowell, B. B., Elmquist, J. K., and Zigman, J. M. (2009). Characterization of a novel ghrelin cell reporter mouse. Regulatory Peptides 155, 91-98.

(301) Kirchner, H., Tong, J., Tschop, M. H., and Pfluger, P. T. (2010). Ghrelin and PYY in the regulation of energy balance and metabolism: lessons from mouse mutants., eng Am J Physiol Endocrinol Metab 298, E909-19.

(302) Szlachcic, A., Brzozowski, T., Majka, J., Pajdo, R., Konturek, P. C., Pawlik, M., Kwiecien, S., Drozdowicz, D., Bielanski, W., Konturek, S. J., and Pawlik, W. W. (2010). Involvement of orexigenic peptides in the mechanism of gastric mucosal integrity and healing of chronic gastric ulcers., eng Curr Pharm Des $16,1214-1223$.

(303) Kern, A., Albarran-Zeckler, R., Walsh, H. E., and Smith, R. G. (2012). ApoGhrelin Receptor Forms Heteromers with DRD2 in Hypothalamic Neurons and Is Essential for Anorexigenic Effects of DRD2 Agonism. Neuron 73, 317332.

(304) Schellekens, H., De Francesco, P. N., Kandil, D., Theeuwes, W. F., McCarthy, T., van Oeffelen, W. E. P. A., Perelló, M., Giblin, L., Dinan, T. G., and Cryan, J. F. (2015). Ghrelin's Orexigenic Effect Is Modulated via a Serotonin 2C Receptor Interaction. ACS Chemical Neuroscience 6, 1186-1197.

(305) Rediger, A., Piechowski, C. L., Yi, C.-X., Tarnow, P., Strotmann, R., Grüters, A., Krude, H., Schöneberg, T., Tschöp, M. H., Kleinau, G., and Biebermann, H. (2011). Mutually Opposite Signal Modulation by Hypothalamic Heterodimerization of Ghrelin and Melanocortin-3 Receptors. Journal of Biological Chemistry 286, 39623-39631.

(306) Wellman, M., and Abizaid, A. (2015). Growth Hormone Secretagogue Receptor Dimers: A New Pharmacological Target. Eneuro 2, DOI: 10.1523 / eneuro.0053-14. 2015. 